\title{
Efficient Slave-Boson Approach for Multiorbital Two-Particle Response Functions and Superconductivity
}

\author{
Tsung-Han Lee, ${ }^{1, *}$ Nicola Lanatà ${ }^{2,3}$ Minjae Kim, ${ }^{1,4}$ and Gabriel Kotliar ${ }^{1,5}$ \\ ${ }^{1}$ Physics and Astronomy Department, Rutgers University, Piscataway, New Jersey 08854, USA \\ ${ }^{2}$ Department of Physics and Astronomy, Aarhus University, 8000 Aarhus C, Denmark \\ ${ }^{3}$ Nordita, KTH Royal Institute of Technology and Stockholm University, \\ Hannes Alfvéns väg 12, SE-106 91 Stockholm, Sweden \\ ${ }^{4}$ Department of Chemistry, Pohang University of Science and Technology (POSTECH), \\ Pohang 37673, Korea \\ ${ }^{5}$ Condensed Matter Physics and Materials Science Department, \\ Brookhaven National Laboratory, Upton, New York 11973, USA
}

(Received 12 April 2021; revised 1 July 2021; accepted 23 August 2021; published 29 November 2021)

\begin{abstract}
We develop an efficient approach for computing two-particle response functions and interaction vertices for multiorbital strongly correlated systems based on the rotationally invariant slave-boson framework. The method is applied to the degenerate three-orbital Hubbard-Kanamori model for investigating the origin of the $s$-wave orbital antisymmetric spin-triplet superconductivity in Hund's metal regime, previously found in the dynamical mean-field theory studies. By computing the pairing interaction considering the particleparticle and the particle-hole scattering channels, we identify the mechanism leading to the pairing instability around Hund's metal crossover arises from the particle-particle channel, which contains the local electron pair fluctuation between different particle-number sectors of the atomic Hilbert space. On the other hand, the particle-hole spin fluctuations induce the $s$-wave pairing instability before entering Hund's regime. Our approach paves the way for investigating the pairing mechanism in realistic correlated materials.
\end{abstract}

DOI: 10.1103/PhysRevX.11.041040

\section{INTRODUCTION}

Slave-boson approaches are among the most widely used theories for describing strongly correlated systems [1-7]. In particular, the saddle-point approximation of the slaveboson method provides a reliable description of the local correlation effects, while requiring a relatively low computational cost compared to dynamical mean-field theory (DMFT) [8]. The development of the rotationally invariant slave-boson (RISB) saddle-point approximation [9], equivalent to the Gutzwiller approximation (GA) [10,11], has also been extended to realistic multiorbital systems, in combination with density functional theory [12,13], uncovering many intriguing phenomena, including the selectiveMott transition [7,14,15], Hund's metal behavior [16-19], valence fluctuations, and correlation induced topological materials [20-22].

\footnotetext{
*Corresponding author. t1596@physics.rutgers.edu

Published by the American Physical Society under the terms of the Creative Commons Attribution 4.0 International license. Further distribution of this work must maintain attribution to the author(s) and the published article's title, journal citation, and DOI.
}

Subject Areas: Strongly Correlated Materials, Superconductivity
Recently, RISB has been reformulated as a quantum embedding theory, where the interacting lattice problem is mapped to an impurity problem coupled to a self-consistently determined environment [21], similar to DMFT and density matrix embedding theory (DMET) $[8,23,24]$. In particular, the RISB saddle-point equations are equivalent to the "noninteracting bath" DMET (NIB-DMET) selfconsistent equations when setting the quasiparticle renormalization matrix to unity and enforcing an additional constraint on the structure of the physical density matrix $[25,26]$. In addition, the two methods, originally proposed for describing the ground state or low-temperature properties, have been extended to study the finite-temperature effects, the nonequilibrium dynamics, the excited states, and the single-particle spectral functions in correlated systems [27-33].

So far, RISB is mostly used for investigating the singleparticle spectral functions and the static local observables. However, the two-particle response functions and the corresponding interaction vertices are also important for explaining the emergent phenomena in correlated materials, e.g., the spin-fluctuation-mediated pairing in unconventional superconductors [34]. Therefore, it is important to extend RISB to study these quantities. Indeed, it is possible to compute the two-particle response functions with the 
Gaussian fluctuation approach around the slave-boson saddle point [3,4,35-44]. However, the technique has so far been restricted to the single-orbital Hubbard model. On the other hand, the development of the time-dependent Gutzwiller approximation has been extended to multiorbital systems and applied to the two-orbital Hubbard model for spin susceptibilities [45-51]. To the best of our knowledge, the theories have not been generalized to compute arbitrary two-particle response functions and quasiparticle interaction vertices for multiorbital systems.

In this work, we develop an efficient approach to compute general susceptibilities and quasiparticle interaction vertices based on fluctuation around the RISB saddle point, allowing a diagrammatic analysis for the pairing mechanism. We apply our method to the degenerate threeorbital Hubbard-Kanamori model to investigate the origin of the $s$-wave orbital-antisymmetric spin-triplet pairing instability in Hund's metal regime, previously found in the DMFT and GA studies [52-55]. We show that, in agreement with DMFT [53], our approach captures the $s$-wave spin-triplet pairing instability around Hund's metal crossover. By investigating the pairing interaction considering the particleparticle and the particle-hole scattering channels, we identify that the mechanism leading to the local $s$-wave orbitalantisymmetric spin-triplet pairing arises from the particleparticle channel, which contains the local electron pair fluctuation between different particle-number sectors of the local Hilbert space. Interestingly, the particle-hole spin-fluctuation mechanism for the $s$-wave pairing, considered also in previous works $[53,56,57]$, induces the $s$-wave pairing instability slightly before entering Hund's regime. Possible applications of our formalism to NIB-DMET are also discussed.

\section{MODEL}

We consider the following generic multiorbital HubbardKanamori model:

$$
\hat{H}=\sum_{\mathbf{k} \alpha \beta \sigma} \epsilon_{\mathbf{k} \alpha \beta} d_{\mathbf{k} \alpha \sigma}^{\dagger} d_{\mathbf{k} \beta \sigma}+\sum_{i} \hat{H}_{\mathrm{loc}}\left[\left\{d_{i \alpha \sigma}^{\dagger}, d_{i \alpha \sigma}\right\}\right],
$$

where $\alpha$ is the orbital index, $\sigma$ is the spin index, $i$ is the unitcell label, and $\mathbf{k}$ is the momentum conjugate to $i$. As a proof of principle and for pedagogical reasons, we assume a three-orbital degenerate model with the energy dispersion of a two-dimensional square lattice with the nearestneighbor hopping:

$$
\epsilon_{\mathbf{k} \alpha \beta}=-2 t\left[\cos \left(\mathbf{k}_{x}\right)+\cos \left(\mathbf{k}_{y}\right)\right] \delta_{\alpha, \beta},
$$

where $\alpha \in\{1,2,3\}$, and we set $t=1$ as the energy unit. However, we note that our formalism applies to multiorbital Hubbard models with a general hopping matrix and an arbitrary number of orbitals. The term $H_{\text {loc }}$ represents the following operator:

$$
\begin{aligned}
& \hat{H}_{\mathrm{loc}}\left[\left\{d_{i, \alpha}^{\dagger}, d_{i \alpha}\right\}\right] \\
& =U \sum_{\alpha} n_{i \alpha \uparrow} n_{i \alpha \downarrow}+U^{\prime} \sum_{\alpha<\alpha^{\prime}, \sigma} n_{i \alpha \sigma} n_{i \alpha^{\prime} \sigma} \\
& \quad+\left(U^{\prime}-J\right) \sum_{\alpha<\alpha^{\prime}, \sigma} n_{i \alpha \sigma} n_{i \alpha^{\prime} \sigma}-J \sum_{\alpha<\alpha^{\prime}}\left(d_{i \alpha \uparrow}^{\dagger} d_{i \alpha \downarrow} d_{i \alpha^{\prime} \downarrow}^{\dagger} d_{i \alpha^{\prime} \uparrow}\right. \\
& \left.\quad+d_{i \alpha \uparrow}^{\dagger} d_{i \alpha \downarrow}^{\dagger} d_{i \alpha^{\prime} \uparrow} d_{i \alpha^{\prime} \downarrow}+\text { H.c. }\right)-\mu_{0} \sum_{\alpha \sigma} n_{\alpha \sigma},
\end{aligned}
$$

which contains the Kanamori interaction [58] in the cubicharmonic basis. The first term is the intraorbital Coulomb interaction, the second term and the third term are the interorbital Coulomb interaction, and the last term contains the spin-flip and the pair hopping interaction. Throughout our paper, we assume the rotationally invariant condition $U^{\prime}=$ $U-2 J$ and set $J=U / 4$. Note that, with this choice of parameters, the bare orbital-antisymmetric spin-triplet pairing interaction is repulsive, i.e., $U^{\prime}-J>0$. The electron occupancy is controlled by the chemical potential $\mu_{0}$.

Because of the $O(3) \otimes S U(2)$ symmetry in the degenerate three-orbital model, the orbital-antisymmetric spin-triplet pairing channels [59-61] are related to each other by a rotation in the orbital and the spin space. Consequently, we focus on the pairing fluctuation in one of the orbitalantisymmetric spin-triplet pairing channels:

$$
\hat{\mathcal{O}}_{\mathrm{P}}=\sum_{\alpha \beta} \sum_{\sigma \sigma^{\prime}}\left[\lambda_{6}\right]_{\alpha \beta}\left[-i \sigma_{y} \sigma_{z}\right]_{\sigma \sigma^{\prime}} d_{i, \alpha \sigma}^{\dagger} d_{i, \beta \sigma^{\prime}}^{\dagger}
$$

Similarly, we have the following independent operators for the charge, spin, orbital, and spin-orbital fluctuation channels:

$$
\hat{\mathcal{O}}_{s}= \begin{cases}\sum_{\alpha \beta}\left[\lambda_{0}\right]_{\alpha \beta}\left[\sigma_{0}\right]_{\sigma \sigma^{\prime}} d_{i, \alpha \sigma}^{\dagger} d_{i, \beta \sigma^{\prime}} & s=\mathrm{ch} \\ \sum_{\alpha \beta}\left[\lambda_{0}\right]_{\alpha \beta}\left[\sigma_{z}\right]_{\sigma \sigma^{\prime}} d_{i, \alpha \sigma}^{\dagger} d_{i, \beta \sigma^{\prime}} & s=\mathrm{sp} \\ \sum_{\alpha \beta}\left[\lambda_{4}\right]_{\alpha \beta}\left[\sigma_{0}\right]_{\sigma \sigma^{\prime}} d_{i, \alpha \sigma}^{\dagger} d_{i, \beta \sigma^{\prime}} & s=\mathrm{orb} \\ \sum_{\alpha \beta}\left[\lambda_{4}\right]_{\alpha \beta}\left[\sigma_{z}\right]_{\sigma \sigma^{\prime}} d_{i, \alpha \sigma}^{\dagger} d_{i, \beta \sigma^{\prime}} & s=\mathrm{so} \\ \sum_{\alpha \beta}\left[\lambda_{1}\right]_{\alpha \beta}\left[\sigma_{0}\right]_{\sigma \sigma^{\prime}} d_{i, \alpha \sigma}^{\dagger} d_{i, \beta \sigma^{\prime}} & s=\text { orb }^{*} \\ \sum_{\alpha \beta}\left[\lambda_{1}\right]_{\alpha \beta}\left[\sigma_{z}\right]_{\sigma \sigma^{\prime}} d_{i, \alpha \sigma}^{\dagger} d_{i, \beta \sigma^{\prime}} & s=\mathrm{so}^{*},\end{cases}
$$

where we label the fluctuation channels by $s \in$ $\{\mathrm{ch}, \mathrm{sp}$, orb, so, orb*, so*, P $\}$ throughout the paper. Here, $\lambda_{0}$ is the $3 \times 3$ identity matrix and $\lambda_{i}$ are the Gell-Mann matrices (see the Appendix A), while $\sigma_{0}$ is the $2 \times 2$ identity matrix and $\sigma_{i}(i=x, y, z)$ are the Pauli matrices. 


\section{METHOD}

Our fluctuation approach around the RISB normal-state saddle point is entirely encoded in the following Lagrange function [62] (see Appendix B):

$\mathcal{L}\left[|\Phi\rangle, \mathbf{R}, \boldsymbol{\Lambda} ; \mathbf{D}, \boldsymbol{\Lambda}^{c}, E^{c}, \boldsymbol{\Delta}\right]$

$=\mathcal{L}_{\mathrm{qp}}[\mathbf{R}, \boldsymbol{\Lambda}]+\mathcal{L}_{\mathrm{emb}}\left[\mathbf{D}, \boldsymbol{\Lambda}^{c},|\Phi\rangle, E^{c}\right]+\mathcal{L}_{\text {mix }}\left[\mathbf{D}, \mathbf{R}, \boldsymbol{\Lambda}^{c}\right]$,

where

$$
\begin{gathered}
\mathcal{L}_{\mathrm{qp}}[\mathbf{R}, \boldsymbol{\Lambda}]=\frac{-T}{N} \frac{1}{2} \sum_{\mathbf{k}_{1} \mathbf{k}_{2} \omega_{n}} \operatorname{Tr} \log \left[-i \omega_{n}+H_{\mathbf{k}_{1} \mathbf{k}_{2}}^{\mathrm{qp}}\right] e^{i \omega_{n} 0^{+}}, \\
\mathcal{L}_{\mathrm{emb}}\left[\mathbf{D}, \Lambda^{c},|\Phi\rangle, E^{c}\right]=\sum_{i}\left\langle\Phi_{i}\left|\hat{H}_{i, \mathrm{emb}}\left[\mathbf{D}_{i}, \boldsymbol{\Lambda}_{i}^{c}\right]\right| \Phi_{i}\right\rangle \\
+E_{i}^{c}\left(\left\langle\Phi_{i} \mid \Phi_{i}\right\rangle-1\right),
\end{gathered}
$$

$$
\begin{aligned}
\mathcal{L}_{\text {mix }}\left[\mathbf{D}, \mathbf{R}, \boldsymbol{\Lambda}^{c}\right]= & -\sum_{i}\left[\frac{1}{2} \sum_{a b}\left(\left[\boldsymbol{\Lambda}_{i}\right]_{a b}+\left[\boldsymbol{\Lambda}_{i}^{c}\right]_{a b}\right)\left[\boldsymbol{\Delta}_{i}\right]_{a b}\right. \\
& \left.+\sum_{a c \alpha}\left(\left[\mathbf{D}_{i}\right]_{a \alpha}\left[\mathbf{R}_{i}\right]_{c \alpha}\left[\boldsymbol{\Delta}_{i}\left(1-\boldsymbol{\Delta}_{i}\right)\right]_{c a}^{1 / 2}+\text { c.c. }\right)\right] .
\end{aligned}
$$

Equation (7) encodes the contribution of the so-called "quasiparticle fermionic" degrees of freedom. Specifically, the matrix

$$
\left[H_{\mathbf{k}_{1} \mathbf{k}_{2}}^{\mathrm{qp}}\right]_{a b}=\frac{1}{N} \sum_{\mathbf{k}}\left[\mathbf{R}_{\mathbf{k}_{1}-\mathbf{k}} \tilde{\mathbf{k}}_{\mathbf{k}} \mathbf{R}_{\mathbf{k}_{2}-\mathbf{k}}^{\dagger}\right]_{a b}+\left[\boldsymbol{\Lambda}_{\mathbf{k}_{1}-\mathbf{k}_{2}}\right]_{a b},
$$

with the hopping term in the Nambu basis,

$$
\tilde{\mathbf{\epsilon}}_{\mathbf{k}}=\left(\begin{array}{cc}
\epsilon_{\mathbf{k}} & 0 \\
0 & -\epsilon_{-k}^{*}
\end{array}\right)
$$

characterizes the "quasiparticle Hamiltonian":

$$
\hat{H}^{\mathrm{qp}}=\sum_{\mathbf{k}_{1}, \mathbf{k}_{2}}\left[H_{\mathbf{k}_{1} \mathbf{k}_{2}}^{\mathrm{qp}}\right]_{a b} \Psi_{\mathbf{k}_{1} a}^{\dagger} \Psi_{\mathbf{k}_{2} b},
$$

where $\Psi_{\mathbf{k}}^{\dagger}=\left(f_{\mathbf{k} 1 \uparrow}^{\dagger} f_{\mathbf{k} 1 \downarrow}^{\dagger} \ldots f_{\mathbf{k} M \uparrow}^{\dagger} f_{\mathbf{k} M \downarrow}^{\dagger} f_{\mathbf{k} 1 \uparrow} f_{\mathbf{k} 1 \downarrow} \ldots f_{\mathbf{k} M \uparrow} f_{\mathbf{k} M \downarrow}\right)$ is a Nambu spinor, $f_{\mathbf{k} a \sigma}$ are the fermionic quasiparticle modes, and $M$ is the total number of orbitals. The matrix $\mathbf{R}$ is the so-called "quasiparticle renormalization matrix," and $\boldsymbol{\Lambda}$ is a matrix of Lagrange multipliers enforcing the RISB constraints $[9,62]$ :

$$
\left[\boldsymbol{\Delta}_{i}\right]_{a b} \equiv\left\langle\Psi_{i a}^{\dagger} \Psi_{i b}\right\rangle_{T},
$$

where $\boldsymbol{\Delta}_{i}$ corresponds to the local quasiparticle density matrices [14], and the symbol $\langle\ldots\rangle_{T}$ denotes the thermal average of the noninteracting quasiparticle Hamiltonian $\hat{H}^{\mathrm{qp}}$ at temperature $T$.
The second term $\mathcal{L}_{\text {emb }}$ [Eq. (8)] encodes the contribution of the slave-boson amplitudes, which we expressed here directly in terms of the corresponding "quantum embedding" states $\left|\Phi_{i}\right\rangle$ and interacting embedding Hamiltonians [21] (see Appendix B 1):

$$
\begin{aligned}
\hat{H}_{i, \mathrm{emb}}= & H_{i, \mathrm{loc}}\left[\left\{\hat{d}_{i \alpha}^{\dagger}, \hat{d}_{i \alpha}\right\}\right]+\left(\sum_{a \alpha b} \mathbf{D}_{i a \alpha} \hat{\Xi}_{i \alpha}^{\dagger} \hat{\Psi}_{i b} \bar{I}_{b a}+\text { H.c. }\right) \\
& +\sum_{a b c d} \frac{1}{2} \boldsymbol{\Lambda}_{i a b}^{c} \bar{I}_{b c} \hat{\Psi}_{i c}^{\dagger} \hat{\Psi}_{i d} \bar{I}_{d a},
\end{aligned}
$$

where $\hat{\Xi}_{i}^{\dagger}=\left(\hat{d}_{i 1 \uparrow}^{\dagger} \hat{d}_{i 1 \downarrow}^{\dagger} \ldots \hat{d}_{i M \uparrow}^{\dagger} \hat{d}_{i M \downarrow}^{\dagger} \hat{d}_{i 1 \uparrow} \hat{d}_{i 1 \downarrow} \ldots \hat{d}_{i M \uparrow} \hat{d}_{i M \downarrow}\right)$ is the impurity Nambu spinor and $\hat{\Psi}_{i}^{\dagger}=\left(\hat{f}_{i 1 \uparrow}^{\dagger} \hat{f}_{i 1 \downarrow}^{\dagger} \ldots\right.$ $\left.\hat{f}_{i M \uparrow}^{\dagger} \hat{f}_{i M \downarrow}^{\dagger} \hat{f}_{i 1 \uparrow} \hat{f}_{i 1 \downarrow} \ldots \hat{f}_{i M \uparrow} \hat{f}_{i M \downarrow}\right)$ is the Nambu spinor for the bath orbitals. The matrix

$$
\bar{I}=\left(\begin{array}{cc}
1 & 0 \\
0 & -1
\end{array}\right)
$$

is the sign exchange matrix generated from the embedding mapping (see Appendix B 1), where 1 is the $2 M \times 2 M$ identity matrix. The variable $E_{i}^{c}$ is a Lagrange multiplier enforcing the normalization of $\left|\Phi_{i}\right\rangle$ :

$$
\left\langle\Phi_{i} \mid \Phi_{i}\right\rangle \equiv 1
$$

The matrix $\boldsymbol{\Lambda}_{i}^{c}$, which describes the embedding Hamiltonian bath potential, is a matrix of Lagrange multipliers enforcing the RISB constraints:

$$
\left[\boldsymbol{\Delta}_{i}\right]_{a b} \equiv\left\langle\Phi_{i}\left|\hat{\Psi}_{i b} \hat{\Psi}_{i a}^{\dagger}\right| \Phi_{i}\right\rangle
$$

The matrix $\mathbf{D}_{i}$, which describes the hybridization between the impurity and the bath orbitals, is a matrix of Lagrange multipliers, enforcing the definition of the renormalization matrix $[9,14,62]$

$$
\mathbf{R}_{i a \alpha}=\sum_{b}\left\langle\Phi_{i}\left|\hat{\Xi}_{i \alpha}^{\dagger} \hat{\Psi}_{i b}\right| \Phi_{i}\right\rangle\left[\boldsymbol{\Delta}_{i}\left(1-\boldsymbol{\Delta}_{i}\right)\right]_{b a}^{-\frac{1}{2}}
$$

The third term $\mathcal{L}_{\text {mix }}$ [Eq. (9)] contains the Lagrange multipliers from both $\mathcal{L}_{\text {qp }}$ and $\mathcal{L}_{\text {emb }}$.

All physical observables can be obtained from the above variational variables at the saddle-point solution of Eq. (6). The total energy is equal to the Lagrange function [Eq. (6)] evaluated at the saddle point. The expectation value of generic local operators $\hat{\mathcal{O}}_{i}\left[\left\{d_{i \alpha}, d_{i \alpha}^{\dagger}\right\}\right]$ is determined from

$$
\left\langle\hat{\mathcal{O}}_{i}\left[\left\{d_{i \alpha}, d_{i \alpha}^{\dagger}\right\}\right]\right\rangle \equiv\left\langle\Phi_{i}\left|\hat{\mathcal{O}}_{i}\left[\left\{\hat{d}_{i \alpha}, \hat{d}_{i \alpha}^{\dagger}\right\}\right]\right| \Phi_{i}\right\rangle .
$$

In particular, the local (physical) single-particle density matrix is obtained from 


$$
\rho_{i, \alpha \beta} \equiv\left\langle\Phi_{i}\left|\hat{\Xi}_{i \alpha}^{\dagger} \hat{\Xi}_{i \beta}\right| \Phi_{i}\right\rangle .
$$

The quasiparticle weight is determined from the $\mathbf{R}$ matrix through $Z_{i}=\mathbf{R}_{i}^{\dagger} \mathbf{R}_{i}$.

Note that within the context of NIB-DMET, Eq. (12) corresponds to the so-called "low-level mean-field" Hamiltonian when setting $\mathbf{R}=I$, and $\boldsymbol{\Lambda}$ is termed the "correlation potential." Equation (14) corresponds to the so-called "high-level many-body Hamiltonian" in NIBDMET, where the two-particle interaction on the bath orbitals is set to zero [24].

\section{A. Parametrization of the single-particle matrices}

To enforce the symmetry conditions of the Lagrange function, we introduce the following parametrization of the renormalization matrix $\mathbf{R}_{i}$ and the Lagrange multipliers $\boldsymbol{\Lambda}_{i}$, $\boldsymbol{\Delta}_{i}, \mathbf{D}_{i}$, and $\boldsymbol{\Lambda}_{i}^{c}[14]$ :

$$
\begin{gathered}
\mathbf{R}_{i}=\sum_{s} r_{i, s} \tilde{\mathbf{h}}_{s}, \\
\boldsymbol{\Lambda}_{i}=\sum_{s} l_{i, s} \mathbf{h}_{s}, \\
\boldsymbol{\Delta}_{i}=\frac{1}{2} \mathbf{1}+\sum_{s} d_{i, s} \mathbf{h}_{s}^{t}, \\
\mathbf{D}_{i}=\sum_{s} D_{i, s} \tilde{\mathbf{h}}_{s}, \\
\boldsymbol{\Lambda}_{i}^{c}=\sum_{s} l_{i, s}^{c} \mathbf{h}_{s},
\end{gathered}
$$

where $\mathbf{1}$ is the $4 M \times 4 M$ identity matrix, and $\mathbf{h}_{s}$ and $\tilde{\mathbf{h}}_{s}$ are the symmetry-adapted matrix basis of the above singleparticle matrices. The structure of the matrix bases $\mathbf{h}_{s}$ and $\tilde{\mathbf{h}}_{s}$ is determined from the group symmetry analysis of the model in the presence of the fluctuating operators [e.g., Eqs. (4) and (5)] [14]. This parametrization allows us to classify the fluctuations of the variational parameters $\left(r_{s}, l_{s}\right.$, etc.) to a specific symmetry channel $s$, associated to $\mathbf{h}_{s}$ and $\tilde{\mathbf{h}}_{s}$. For example, in the degenerate three-orbital Hubbard-Kanamori model, the $\mathbf{h}_{s}$ and $\tilde{\mathbf{h}}_{s}$ (see Appendix C 2) are associated to the fluctuation channels $s \in\left\{\mathrm{ch}, \mathrm{sp}\right.$, orb, so, orb*, $\left.\mathrm{so}^{*}, \mathrm{P}\right\}$ in Eqs. (4) and (5). In addition, for computing the susceptibility of a given channel $s$, the embedding wave function $\left|\Phi_{i}\right\rangle$ has to break the corresponding symmetry; e.g., the particlenumber conservation of $\left|\Phi_{i}\right\rangle$ has to be broken for the pairing susceptibility calculations.

For later convenience, we introduce the following vector of parameters:

$$
\begin{array}{r}
\mathbf{x}_{i}=\left(r_{i, \mathrm{ch}}, l_{i, \mathrm{ch}}, d_{i, \mathrm{ch}}, D_{i, \mathrm{ch}}, l_{i, \mathrm{ch}}^{c}, \ldots, r_{i, s}, l_{i, s},\right. \\
\left.d_{i, s}, D_{i, s}, l_{i, s}^{c}, \ldots, r_{i, \mathrm{P}}, l_{i, \mathrm{P}}, d_{i, \mathrm{P}}, D_{i, \mathrm{P}}, l_{i, \mathrm{P}}^{c}\right),
\end{array}
$$

and assume that all of its entries are real, which is sufficient for static quantities (e.g., static susceptibilities and Landau parameters $[39,42])$. Note that our assumption of real variables is applicable for our model without spin-orbit coupling. The generalization to spin-orbit coupled systems can be straightforwardly obtained using the same procedure proposed in this work, by including in the Lagrangian also the imaginary part of $\mathbf{R}$ and $\mathbf{D}$.

\section{B. Saddle-point approximation}

The first step of our fluctuation approach is to determine the normal-state saddle-point solution without any ordering. We assume a spatially homogeneous saddle-point solution, where $\mathbf{x}_{i}$ does not depend on $i$.

Performing the partial derivatives of Eq. (48) with respect to $\mathbf{x}$, we arrive at the following saddle-point equations:

$$
\begin{gathered}
{[\mathbf{\Delta}]_{a b}=\frac{1}{N} \sum_{\mathbf{k}}\left[f_{T}\left(H_{\mathbf{k}}^{\mathrm{qp}}\right)\right]_{b a}} \\
{[\boldsymbol{\Delta}(1-\boldsymbol{\Delta})]_{a c}^{1 / 2} \mathbf{D}_{c a}=\frac{1}{N} \frac{1}{2} \sum_{\mathbf{k}}\left[\tilde{\mathbf{\epsilon}}_{\mathbf{k}} R^{\dagger} f_{T}\left(H_{\mathbf{k}}^{\mathrm{qp}}\right)\right]_{\alpha a}} \\
\sum_{c b \alpha} \partial_{d_{s}}[\boldsymbol{\Delta}(1-\boldsymbol{\Delta})]_{c b}^{1 / 2}[\mathbf{D}]_{b \alpha}[\mathbf{R}]_{c \alpha}+\text { c.c. }+\frac{1}{2}\left[l_{s}+l_{s}^{c}\right]=0 \\
{\left[\hat{H}^{(1)}\right]_{a b} \equiv\left\langle\Phi\left|\bar{I}_{b c} \hat{\Psi}_{c} \hat{\Psi}_{d}^{\dagger} \bar{I}_{d a}\right| \Phi\right\rangle-[\mathbf{\Delta}]_{a b}=0} \\
{\left[\mathcal{F}^{(2)}\right]_{\alpha a} \equiv\left\langle\Phi\left|\hat{\Xi}_{\alpha}^{\dagger} \hat{\Psi}_{b} \bar{I}_{b a}\right| \Phi\right\rangle-\mathbf{R}_{c \alpha}[\mathbf{\Delta}(1-\boldsymbol{\Delta})]_{c a}^{1 / 2}=0}
\end{gathered}
$$

where $f_{T}$ is the Fermi function and $H_{\mathbf{k}}^{\mathrm{qp}}=\mathbf{R} \tilde{\epsilon}_{\mathbf{k}} \mathbf{R}^{\dagger}+\boldsymbol{\Lambda}$ is the saddle-point quasiparticle Hamiltonian. Equations (27)-(32) can be solved numerically by utilizing quasi-Newton methods $[14,21]$. Note that our saddle-point equations yield consistent results compared to the formalism in Ref. [62].

It is also interesting to point out that Eqs. (27)-(32) are equivalent to the NIB-DMET self-consistent equations when setting the renormalization matrix to unity $\mathbf{R}=I$ and enforcing the so-called "quasiparticle constraint" that we will introduce later in Sec. IV [25].

Given the saddle-point solution in the normal phase, we want to compute the corresponding susceptibilities. This will be accomplished using the approach described below.

\section{Calculation of susceptibilities}

Here, we describe the formalism for calculating the susceptibilities in multiorbital systems within the RISB framework. For concreteness, we focus on uniform susceptibilities in this section, where $\mathbf{x}_{i}$ is independent of $i$, and we suppress the $i$ index in the following derivation. 
The generalization to susceptibilities with finite momentum transfer is described in Sec. IV.

Let us consider the RISB Lagrange function [Eq. (6)] in the presence of a local perturbation, proportional to a generic operator $\hat{\mathcal{O}}$ :

$\mathcal{L}\left[\xi, \mathbf{x}, \Phi, E^{c}\right]=\mathcal{L}_{\mathrm{qp}}[\mathbf{x}]+\mathcal{L}_{\text {mix }}[\mathbf{x}]+\mathcal{L}_{\mathrm{emb}}\left[\xi, \mathbf{x}, \Phi, E^{c}\right]$,

where we have modified the embedding part of the Lagrangian to

$$
\begin{aligned}
\mathcal{L}_{\mathrm{emb}}\left[\xi, \mathbf{x}, \Phi, E^{c}\right]= & \sum_{i}\left\langle\Phi(\mathbf{x})\left|\hat{H}_{\mathrm{emb}}[\mathbf{x}]-\xi \hat{\mathcal{O}}\right| \Phi(\mathbf{x})\right\rangle \\
& +E^{c}(1-\langle\Phi(\mathbf{x}) \mid \Phi(\mathbf{x})\rangle)
\end{aligned}
$$

which was obtained by adding a field $\xi$ coupled to $\hat{\mathcal{O}}$ in the embedding Hamiltonian of Eq. (14) and by expressing the variational parameters in terms of the vector $\mathbf{x}$; see Eq. (26).

To calculate the linear response of the system to the perturbation $\hat{\mathcal{O}}$, we need to evaluate how the saddle-point variational parameters $\mathbf{x}$ of Eq. (33) evolve as a function of $\xi$. For this purpose, it is convenient to introduce the following functional:

$$
\begin{aligned}
\Omega[\xi, \mathbf{x}]= & \mathcal{L}_{\mathrm{qp}}[\mathbf{x}]+\mathcal{L}_{\text {mix }}[\mathbf{x}] \\
& +\mathcal{L}_{\mathrm{emb}}\left[\xi, \Phi(\xi, \mathbf{x}), E^{c}(\xi, \mathbf{x})\right],
\end{aligned}
$$

where $|\Phi(\xi, \mathbf{x})\rangle$ and $E^{c}(\xi, \mathbf{x})$ are the ground state of $\hat{H}_{\mathrm{emb}}$ and its eigenvalue, respectively; see Eq. (30). Within these definitions, the saddle-point solution of $\mathbf{x}$ for a given $\xi$, which we call $\mathbf{x}(\xi)$, is defined by

$$
\left.\partial_{\mathbf{x}} \Omega[\xi, \mathbf{x}]\right|_{[\xi, \mathbf{x}(\xi)]}=0,
$$

and the linear response for the operator $\hat{\mathcal{O}}$ is given by the following equation (see Appendix D for derivation):

$$
\chi_{\mathcal{O O}}=\chi_{\mathcal{O O}}^{\mathrm{emb}}+\sum_{\mu} \chi_{\mu \mathcal{O}}^{\mathrm{emb}} \mathcal{M}_{\mu \nu}^{-1} \chi_{\nu \mathcal{O}}^{\mathrm{emb}}
$$

where we introduced the susceptibilities:

$$
\begin{aligned}
& \chi_{\mathcal{O O}}^{\mathrm{emb}}=\left.\partial_{\xi}\langle\Phi(\xi, \mathbf{x})|\hat{\mathcal{O}}| \Phi(\xi, \mathbf{x})\rangle\right|_{[\xi=0, \mathbf{x}(\xi=0)]}, \\
& \chi_{\mu \mathcal{O}}^{\mathrm{emb}}=\left.\partial_{x_{\mu}}\langle\Phi(\xi, \mathbf{x})|\hat{\mathcal{O}}| \Phi(\xi, \mathbf{x})\rangle\right|_{[\xi=0, \mathbf{x}(\xi=0)]} .
\end{aligned}
$$

The so-called "fluctuation matrix" is

$$
\mathcal{M}_{\mu \nu}=\left.\partial_{x_{\mu}} \partial_{x_{\nu}} \Omega[\xi, \mathbf{x}]\right|_{[\xi=0, \mathbf{x}(\xi=0)]} .
$$

Here, the indices $\mu$ and $\nu$ run through all the variational variables in Eq. (26), i.e., $r_{s}, l_{s}, d_{s}, D_{s}, l_{s}^{c}$. To keep track of the structure of the fluctuation matrix (where different second-order derivatives are computed through different equations; see Appendix E), from now on we will often use these variational variables as matrix subscripts. For example, $\mathcal{M}_{D_{s}, c_{s}^{\prime}}$ corresponds to the second-order derivatives with respect to $D_{s}$ and $l_{s^{\prime}}^{c}$ [see Eq. (E21)].

It is important to note that $\mathcal{M}$ is not invertible. This is because the functional $\Omega$ is invariant with respect to the gauge transformation [Eq. (H6)], so $\mathcal{M}$ is not unique because of the would-be Goldstone modes. As explained in Appendix I, this redundancy can be systematically resolved by operating a gauge-fixing process that removes the would-be Goldstone modes from the onset [47]. A simpler alternative is to solve the overdetermined linear system [Eq. (40)] by introducing the Moore-Penrose pseudoinverse of the fluctuation matrix, which we indicate as $\overline{\mathcal{M}}^{-1}$. In terms of the pseudoinverse, the susceptibility can be formally expressed as follows:

$$
\chi_{\mathcal{O O}}=\chi_{\mathcal{O O}}^{\mathrm{emb}}+\sum_{\mu \nu} \chi_{\mu \mathcal{O}}^{\mathrm{emb}} \overline{\mathcal{M}}_{\mu \nu}^{-1} \chi_{\nu \mathcal{O}}^{\mathrm{emb}}
$$

Note that Eq. (41) applies for general multiorbital Hubbard models, and the procedure for evaluating each element, Eqs. (38)-(40), is described in Appendix E.

We now discuss the application of our formalism to the degenerate three-orbital Hubbard-Kanamori model. For the considered model, the fluctuation matrix $\mathcal{M}$ reduces to a block-diagonal matrix, constructed by seven $5 \times 5$ matrices shown schematically in Fig. 1 (one for each fluctuation channel $s$ ) because of the orthonormality of the fluctuation basis $\operatorname{Tr}\left[\mathbf{h}_{s} \mathbf{h}_{s^{\prime}}^{\dagger}\right]=\delta_{s s^{\prime}}$. Furthermore, for a given channel $s$, $\chi_{\mu \mathcal{O}}^{\mathrm{emb}}$ [see Eq. (39)] is nonzero only for the components $\mu=D_{s}$ and $l_{s}$. Therefore, Eq. (41), for a given channel $s$, can be further simplified to

$$
\begin{aligned}
\chi_{\mathcal{O}_{s} \mathcal{O}_{s}}= & \chi_{\mathcal{O}_{s} \mathcal{O}_{s}}^{\mathrm{emb}}+\chi_{D_{s} \mathcal{O}_{s}}^{\mathrm{emb}} \overline{\mathcal{M}}_{D_{s} D_{s}}^{-1} \chi_{D_{s} \mathcal{O}_{s}}^{\mathrm{emb}} \\
& +2 \chi_{D_{s} \mathcal{O}_{s}}^{\mathrm{emb}} \overline{\mathcal{M}}_{D_{s} l_{s}^{-1}}^{-1} \chi_{l_{s}^{c} \mathcal{O}_{s}}^{\mathrm{emb}}+\chi_{l_{s}^{c} \mathcal{O}_{s}}^{\mathrm{emb}} \overline{\mathcal{M}}_{l_{s}^{c} c_{s}}^{-1} \chi_{l_{s}^{c} \mathcal{O}_{s}}^{\mathrm{emb}},
\end{aligned}
$$

where $\overline{\mathcal{M}}_{D_{s} D_{s}}^{-1}$ denotes the $\mu=D_{s}$ and $\nu=D_{s}$ components of $\overline{\mathcal{M}}_{\mu \nu}^{-1}$ and similarly applies to $\overline{\mathcal{M}}_{D_{s} l_{s}}^{-1}$ and $\overline{\mathcal{M}}_{l_{s}^{c} l_{s}}^{-1}$. We only

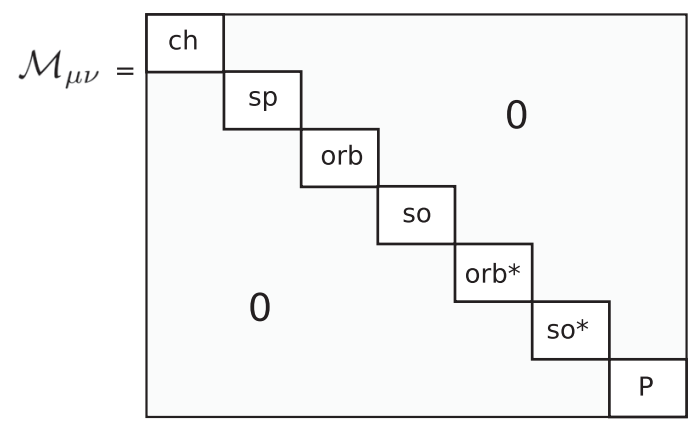

FIG. 1. Schematic representation of the block-diagonalized fluctuation matrix in the charge, spin, orbital, spin-orbital, and pairing sectors for the three-orbital degenerate HubbardKanamori model [see Eqs. (4) and (5)]. 
need to evaluate the $5 \times 5$ fluctuation matrix and its pseudoinversion within each $s$ block to compute the corresponding susceptibility. Note that the block-diagonal structure is not directly applicable to generic systems because of effects such as orbital differentiation or spinorbit coupling. In these cases, one has to compute the full fluctuation matrix for calculating response functions.

\section{FERMI-LIQUID APPROXIMATION AND DIAGRAMMATIC APPROACH}

The Landau Fermi-liquid theory allows one to describe the thermodynamic properties of metals in terms of an effective noninteracting picture. Importantly, this framework applies only to conserved quantities. In particular, since the superconducting order parameter $\hat{\mathcal{O}}_{P}$ does not commute with Eq. (1), the corresponding susceptibility is not rigorously expressible in terms of quasiparticle parameters. Nevertheless, as we show below, within the RISB framework, it is possible to derive an approximate (but accurate) expression for the superconducting susceptibility in terms of the quasiparticle Green's function and interaction vertices. Moreover, the susceptibility can be formulated in terms of the Bethe-Salpeter equation, allowing further diagrammatic analysis for the pairing mechanism.

From the point of view of the RISB methodology, the reason why the superconducting susceptibility cannot be calculated in terms of quasiparticle parameters is that

$K_{s}\left[\Phi_{i}, \Delta_{i}\right]=\sum_{\alpha \beta}\left[\mathbf{h}_{s}\right]_{\alpha \beta}\left(\left\langle\Phi_{i}\left|\Xi_{i \alpha}^{\dagger} \Xi_{i \beta}\right| \Phi_{i}\right\rangle-\Delta_{i \alpha \beta}\right) \neq 0$

for $s=$ P; i.e., the physical density matrix is, in general, not the same as the quasiparticle density matrix.

Here, we propose to modify the spatially inhomogeneous RISB Lagrange function [Eq. (6)] by imposing the constraint

$$
K_{\mathrm{s}}\left[\Phi_{i}, \Delta_{i}\right]=0
$$

which is accomplished by introducing additional Lagrange multipliers $\zeta_{i, s}$ into Eq. (26) so that the $\mathbf{x}$ vector becomes

$$
\begin{aligned}
\mathbf{x}_{i}= & \left(r_{i, \mathrm{ch}}, l_{i, \mathrm{ch}}, d_{i, \mathrm{ch}}, D_{i, \mathrm{ch}}, l_{i, \mathrm{ch}}^{c}, \zeta_{i, \mathrm{ch}}, \ldots, r_{i, s}, l_{i, s},\right. \\
& \left.d_{i, s}, D_{i, s}, l_{i, s}^{c}, \zeta_{i, s}, \ldots, r_{i, \mathrm{P}}, l_{i, \mathrm{P}}, d_{i, \mathrm{P}}, D_{i, \mathrm{P}}, l_{i, \mathrm{P}}^{c}, \zeta_{i, \mathrm{P}}\right) .
\end{aligned}
$$

We also introduce $\mathbf{x}_{\mathbf{q}}$, which is the momentum conjugate to $\mathbf{x}_{i}$.

The Lagrange function now has the following form:

$\mathcal{L}\left[\xi, \mathbf{x}, \Phi, E^{c}\right]=\mathcal{L}_{\mathrm{qp}}\left[\xi_{\mathbf{q}}, \mathbf{x}_{\mathbf{q}}\right]+\mathcal{L}_{\text {mix }}\left[\mathbf{x}_{i}\right]+\mathcal{L}_{\mathrm{emb}}\left[\mathbf{x}_{i}, \Phi_{i}, E_{i}^{c}\right]$

where

$$
\begin{aligned}
\mathcal{L}_{\mathrm{qp}}\left[\xi_{\mathbf{q}}, \mathbf{x}_{\mathbf{q}}\right]=- & \frac{T}{N} \frac{1}{2} \sum_{\omega_{n}} \sum_{\mathbf{k}_{1} \mathbf{k}_{2}} \operatorname{Tr} \log \left[-\mathbf{G}_{\omega_{n}, \mathbf{k}_{1}, \mathbf{k}_{2}}^{-1}[\mathbf{x}, \xi]\right], \quad(47) \\
\mathcal{L}_{\mathrm{emb}}\left[\mathbf{x}_{i}, \Phi_{i}, E_{i}^{c}\right]= & \sum_{i}\left\langle\Phi_{i}\left(\mathbf{x}_{i}\right)\right| \hat{H}_{\mathrm{emb}}\left[\mathbf{x}_{i}\right]+\frac{1}{2} \sum_{\alpha \beta s} \zeta_{i, s} \mathbf{h}_{s, \alpha \beta} \\
& \hat{\Xi}_{i \alpha}^{\dagger} \hat{\Xi}_{i \beta}\left|\Phi_{i}\left(\mathbf{x}_{i}\right)\right\rangle+E_{i}^{c}\left(1-\left\langle\Phi_{i}\left(\mathbf{x}_{i}\right) \mid \Phi_{i}\left(\mathbf{x}_{i}\right)\right\rangle\right),
\end{aligned}
$$

$$
\begin{aligned}
\mathcal{L}_{\text {mix }}\left[\mathbf{x}_{i}\right]= & -\sum_{i}\left[\frac{1}{2} \sum_{a b}\left(\boldsymbol{\Lambda}_{i a b}+\boldsymbol{\Lambda}_{i a b}^{c}+\sum_{s} \zeta_{i s} \mathbf{h}_{s, a b}\right) \boldsymbol{\Delta}_{i a b}\right. \\
& \left.+\sum_{a \alpha c}\left(\mathbf{D}_{i a \alpha} \mathbf{R}_{i c \alpha}[\mathbf{\Delta}(1-\boldsymbol{\Delta})]_{i c a}^{\frac{1}{2}}+\text { c.c. }\right)\right]
\end{aligned}
$$

where we have introduced the physical Green's function

$$
\mathbf{G}_{\omega_{n}, \mathbf{k}_{1}, \mathbf{k}_{2}}[\xi, \mathbf{x}]=\mathbf{R}^{\dagger} \mathbf{G}_{\omega_{n}, \mathbf{k}_{1}, \mathbf{k}_{2}}^{\mathrm{qp}}[\xi, \mathbf{x}] \mathbf{R}
$$

and the quasiparticle Green's function

$$
\left[\mathbf{G}_{\omega_{n}, \mathbf{k}_{1}, \mathbf{k}_{2}}^{\mathrm{qp}}[\xi, \mathbf{x}]\right]_{a b}^{-1}=i \omega_{n}-\left[H_{\mathbf{k}_{1} \mathbf{k}_{2}}^{\mathrm{qp}}[\mathbf{x}]\right]_{a b}+\xi_{\mathbf{k}_{1}-\mathbf{k}_{2}}[\mathcal{O}]_{a b} .
$$

Similar to the previous section, we also introduced a field $\xi_{\mathbf{k}_{1}-\mathbf{k}_{2}}$ coupled to a generic quasiparticle operator $\hat{\mathcal{O}}=$ $\sum_{a b} \Psi_{\mathbf{k}_{1} a}^{\dagger}[\mathcal{O}]_{a b} \Psi_{\mathbf{k}_{2} b}$ into $\mathcal{L}_{\mathrm{qp}}$. This modification allows us to derive momentum-dependent susceptibilities for investigating the finite momentum (commensurate or incommensurate) instabilities. From now on, we refer to Eq. (44) as the "quasiparticle constraint."

Since utilizing the Lagrange equations (47)-(49) amounts to solving the RISB equations (27)-(32) within a reduced variational space, the corresponding solution is an approximation to the original one. In principle, enforcing the constraint [Eq. (44)] does not affect the results for the conserving channels, where the fluctuating operator commutes with the Hamiltonian, e.g., the charge and spin channels. However, it slightly reduces the variational freedom when the constraint is imposed on the nonconserving channel, e.g., the pairing channel. Nevertheless, as we will show, it is always possible to verify a posteriori the accuracy of the approximation by comparison to the formalism without the constraint (see also Appendix J).

It is also interesting to point out that Eq. (44) corresponds to the density matrix mapping constraint in DMET [24]. Therefore, the formalism presented in this section is also applicable to the NIB-DMET by removing the $r_{s}$ sector of the fluctuation basis [Eq. (45)] and setting $\mathbf{R}=I$ [25]. This application is discussed in Appendix N. 


\section{A. Susceptibility: Diagrammatic expression}

Here, we show how the susceptibility evaluated with the quasiparticle constraint can be expressed in terms of the Feynman diagram in perturbation theory.

Following the procedure in Sec. III C, we introduce the following functional:

$$
\begin{aligned}
\Omega[\xi, \mathbf{x}]= & \mathcal{L}_{\mathrm{qp}}\left[\xi_{\mathbf{q}}, \mathbf{x}_{\mathbf{q}}\right]+\mathcal{L}_{\text {mix }}\left[\mathbf{x}_{i}\right] \\
& +\mathcal{L}_{\mathrm{emb}}\left[\Phi\left(\mathbf{x}_{i}\right), E^{c}\left(\mathbf{x}_{i}\right)\right],
\end{aligned}
$$

where now $\mathcal{L}_{\mathrm{qp}}$ depends on the field $\xi_{\mathrm{q}}$. The linear response for a generic operator is given by the following equation:

$$
\begin{aligned}
\chi_{\mathcal{O O}}(\mathbf{q}) & =\left.\frac{T}{2 N} \sum_{\mathbf{k} \omega_{n}} \frac{d}{d \xi_{\mathbf{q}}} \operatorname{Tr}\left[\mathbf{G}_{\omega_{n}, \mathbf{k}+\mathbf{q}, \mathbf{k}}[\xi, \mathbf{x}] \overline{\mathcal{O}}\right]\right|_{[\xi=0, \mathbf{x}(\xi=0)]} \\
& =\chi_{\mathcal{O O}}^{(0)}(\mathbf{q})+\sum_{\mu \nu} \chi_{\mu \mathcal{O}}(\mathbf{q}) \mathcal{M}_{\mu \nu}^{-1}(\mathbf{q}) \chi_{\nu \mathcal{O}}(\mathbf{q}),
\end{aligned}
$$

where the bare susceptibilities are

$$
\begin{gathered}
\chi_{\mathcal{O}}^{(0)}(\mathbf{q})=-\frac{T}{2 N} \sum_{\mathbf{k} \omega_{n}} \operatorname{Tr}\left[\mathbf{G}_{\omega_{n}, \mathbf{k}+\mathbf{q}} \overline{\mathcal{O}} \mathbf{G}_{\omega_{n}, \mathbf{k}} \overline{\mathcal{O}}\right], \\
\chi_{\mu \mathcal{O}}(\mathbf{q})=\left.\frac{T}{2 N} \sum_{\mathbf{k} \omega_{n}} \partial_{x_{\mu, \mathbf{q}}} \operatorname{Tr}\left[\mathbf{G}_{\omega_{n}, \mathbf{k}+\mathbf{q}, \mathbf{k}}[\xi, \mathbf{x}] \overline{\mathcal{O}}\right]\right|_{[\xi=0, \mathbf{x}(\xi=0)]}
\end{gathered}
$$

Note again that $\mu$ runs through all the elements in Eq. (45), and we use the variational parameters as subscripts. We also introduce the saddle-point Green's function $\mathbf{G}_{\omega_{n}, \mathbf{k}}=$ $\mathbf{R}^{\dagger}\left[i \omega_{n}-H_{\mathbf{k}}^{\mathrm{qp}}\right]^{-1} \mathbf{R}$ and $\overline{\mathcal{O}}=[\mathbf{R}]^{-1} \mathcal{O}\left[\mathbf{R}^{\dagger}\right]^{-1}$. The fluctuation matrix $\mathcal{M}$ now depends on momentum $\mathbf{q}$ and has an additional component $\zeta_{s}$ [see Eq. (45)]. The specific form of $\mathcal{M}$ is given in Appendix E. Furthermore, $\mathcal{M}$ is now an invertible matrix because the quasiparticle constraint breaks the gauge symmetry. Note that Eq. (53) applies for generic multiorbital Hubbard models.

We now discuss the application of our approach to the degenerate three-orbital Hubbard-Kanamori model. As described in the previous section, for the degenerate model considered here, $\mathcal{M}$ is a block-diagonal matrix shown schematically in Fig. 1. Also, from Eqs. (4) and (5) and Eqs. (C7)-(C14), we have $\overline{\mathcal{O}}_{s}=\overline{\mathbf{h}}_{s}=[\mathbf{R}]^{-1} \mathbf{h}_{s}\left[\mathbf{R}^{\dagger}\right]^{-1}$ for each fluctuation channel $s$. Therefore, the susceptibility can be simplified to

$$
\begin{aligned}
\chi_{\mathcal{O}_{s} \mathcal{O}_{s}}(\mathbf{q})= & \chi_{\mathcal{O}_{s} \mathcal{O}_{s}}^{(0)}(\mathbf{q})+\chi_{r_{s} \mathcal{O}_{s}}(\mathbf{q}) \mathcal{M}_{r_{s} r_{s}}^{-1}(\mathbf{q}) \chi_{r_{s} \mathcal{O}_{s}}(\mathbf{q}) \\
& +2 \chi_{r_{s} \mathcal{O}_{s}}(\mathbf{q}) \mathcal{M}_{r_{s} l_{s}}^{-1}(\mathbf{q}) \chi_{l_{s} \mathcal{O}_{s}}(\mathbf{q}) \\
& +\chi_{l_{s} \mathcal{O}_{s}}(\mathbf{q}) \mathcal{M}_{l_{s} l_{s}}^{-1}(\mathbf{q}) \chi_{l_{s} \mathcal{O}_{s}}(\mathbf{q})
\end{aligned}
$$

where

$$
\begin{aligned}
& \chi_{r_{s} \mathcal{O}_{s}}(\mathbf{q})=-\frac{T}{2 N} \sum_{\mathbf{k} \omega_{n}} \operatorname{Tr}\left[\mathbf { G } _ { \omega _ { n } , \mathbf { k } + \mathbf { q } } [ \mathbf { R } ] ^ { - 1 } \left[\left(\tilde{\mathbf{h}}_{s} \mathbf{\epsilon}_{\mathbf{k}+\mathbf{q}} \mathbf{R}^{\dagger}\right.\right.\right. \\
&\left.\left.+\mathbf{R \epsilon}_{\mathbf{k}} \tilde{\mathbf{h}}_{s}^{\dagger}\right)\left[\mathbf{R}^{\dagger}\right]^{-1} \mathbf{G}_{\omega_{n}, \mathbf{k}} \overline{\mathbf{h}}_{s}\right], \\
& \chi_{l_{s} \mathcal{O}_{s}}(\mathbf{q})=-\frac{T}{2 N} \sum_{\mathbf{k} \omega_{n}} \operatorname{Tr}\left[\mathbf{G}_{\omega_{n}, \mathbf{k}+\mathbf{q}} \overline{\mathbf{h}}_{s} \mathbf{G}_{\omega_{n}, \mathbf{k}} \overline{\mathbf{h}}_{s}\right] .
\end{aligned}
$$

The $\mathcal{M}_{r_{s} r_{s}}^{-1}(\mathbf{q})$ denotes the $\mu=r_{s}$ and $\nu=r_{s}$ components of $\mathcal{M}_{\mu \nu}^{-1}(\mathbf{q})$ and similarly applies to $\mathcal{M}_{r_{s} l_{s}}^{-1}(\mathbf{q})$ and $\mathcal{M}_{l_{s} l_{s}}^{-1}(\mathbf{q})$. We only need to evaluate the $6 \times 6$ fluctuation matrix and its inversion within each $s$ block to compute the corresponding susceptibility.

To make a connection to perturbation theory, we compare Eq. (56) with the Bethe-Salpeter representation of the susceptibility:

$$
\begin{aligned}
\chi_{\mathcal{O}_{s} \mathcal{O}_{s}}(\mathbf{q})= & \chi_{\mathcal{O}_{s} \mathcal{O}_{s}}^{(0)}(\mathbf{q})-\left(\frac{-T}{2 N}\right)^{2} \sum_{\alpha \beta \gamma \delta} \sum_{\mathbf{k} \mathbf{k}^{\prime}} \sum_{\omega_{n} \omega_{n^{\prime}}}\left[\mathbf{G}_{\omega_{n}, \mathbf{k}} \overline{\mathbf{h}}_{s}\right. \\
& \left.\times \mathbf{G}_{\omega_{n}, \mathbf{k}+\mathbf{q}}\right]_{\beta \alpha} \tilde{\Gamma}_{\alpha \beta \gamma \delta}^{s}\left(\mathbf{k}, \mathbf{k}^{\prime}, \mathbf{q}\right)\left[\mathbf{G}_{\omega_{n^{\prime}}, \mathbf{k}^{\prime}} \overline{\mathbf{h}}_{s} \mathbf{G}_{\omega_{n^{\prime}}, \mathbf{k}^{\prime}+\mathbf{q}}\right]_{\delta \gamma},
\end{aligned}
$$

where $\tilde{\Gamma}_{\alpha \beta \gamma \delta}^{s}\left(\mathbf{k}, \mathbf{k}^{\prime}, \mathbf{q}\right)$ is the (reducible) interaction vertex. To extract the $\tilde{\Gamma}_{\alpha \beta \gamma \delta}^{s}\left(\mathbf{k}, \mathbf{k}^{\prime}, \mathbf{q}\right)$ from Eq. (56), we introduce the following three-leg vertices:

$$
\begin{gathered}
\tilde{\Lambda}_{\alpha \beta r_{s}}(\mathbf{k}, \mathbf{q}) \equiv \frac{1}{2}[\mathbf{R}]_{\alpha a}^{-1}\left[\mathbf{R} \tilde{\epsilon}_{\mathbf{k}+\mathbf{q}} \tilde{\mathbf{h}}_{s}^{\dagger}+\tilde{\mathbf{h}}_{s} \tilde{\epsilon}_{\mathbf{k}} \mathbf{R}^{\dagger}\right]_{a b}\left[\mathbf{R}^{\dagger}\right]_{b \beta}^{-1}, \\
\tilde{\Lambda}_{\alpha \beta l_{s}} \equiv \frac{1}{2}[\mathbf{R}]_{\alpha a}^{-1} \mathbf{h}_{s, a b}\left[\mathbf{R}^{\dagger}\right]_{b \beta}^{-1},
\end{gathered}
$$

such that the susceptibilities can be written as

$$
\begin{array}{r}
\chi_{r_{s} \mathcal{O}_{s}}(\mathbf{q})=-\frac{T}{N} \sum_{\mathbf{k} \omega_{n}} \operatorname{Tr}\left[\mathbf{G}_{\omega_{n}, \mathbf{k}+\mathbf{q}} \tilde{\Lambda}_{r_{s}}(\mathbf{k}, \mathbf{q}) \mathbf{G}_{\omega_{n}, \mathbf{k}} \overline{\mathbf{h}}_{s}\right], \\
\chi_{l_{s} \mathcal{O}_{s}}(\mathbf{q})=-\frac{T}{N} \sum_{\mathbf{k} \omega_{n}} \operatorname{Tr}\left[\mathbf{G}_{\omega_{n}, \mathbf{k}+\mathbf{q}} \tilde{\Lambda}_{l_{s}} \mathbf{G}_{\omega_{n}, \mathbf{k}} \overline{\mathbf{h}}_{s}\right]
\end{array}
$$

Substituting Eqs. (62) and (63) into Eq. (56), we obtain the interaction vertex [see Eq. (59)]:

$$
\begin{aligned}
\tilde{\Gamma}_{\alpha \beta \gamma \delta}^{s}\left(\mathbf{k}, \mathbf{k}^{\prime}, \mathbf{q}\right)= & -4\left(\begin{array}{ll}
\tilde{\Lambda}_{\alpha \beta r_{s}}(\mathbf{k}, \mathbf{q}) & \tilde{\Lambda}_{\alpha \beta l_{s}}
\end{array}\right) \\
& \times\left(\begin{array}{cc}
\mathcal{M}_{r_{s} r_{s}}^{-1}(\mathbf{q}) & \mathcal{M}_{r_{s} l_{s}}^{-1}(\mathbf{q}) \\
\mathcal{M}_{r_{s} l_{s}}^{-1}(\mathbf{q}) & \mathcal{M}_{l_{s} l_{s}}^{-1}(\mathbf{q})
\end{array}\right)\left(\begin{array}{c}
\tilde{\Lambda}_{\gamma \delta r_{s}}\left(\mathbf{k}^{\prime}, \mathbf{q}\right) \\
\tilde{\Lambda}_{\gamma \delta l_{s}}
\end{array}\right),
\end{aligned}
$$

describing the effective interaction between quasiparticles mediated by the bosonic propagator $\mathcal{M}_{\mu \nu}^{-1}$ in the corresponding channel. 
(a)

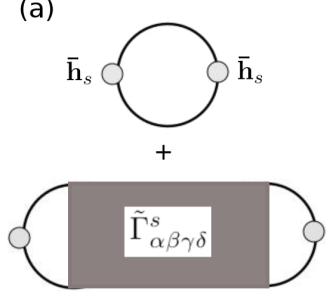

(b)

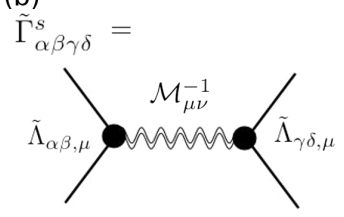

FIG. 2. (a) Diagrammatic representation of the susceptibility [Eq. (59)]. The thick solid line indicates the Nambu fermionic propagator. The gray circle corresponds to the fluctuation basis $\overline{\mathbf{h}}_{s}$, and the gray square corresponds to the quasiparticle interaction vertex $\tilde{\Gamma}_{\alpha \beta \gamma \delta}^{s}$. (b) Diagrammatic representation of the quasiparticle interaction vertex $\tilde{\Gamma}_{\alpha \beta \gamma \delta}^{s}$ [Eq. (64)]. The double wavy line corresponds to the dressed bosonic propagator containing the infinite summation of the particle-particle or the particle-hole fermionic bubbles. The black circle denotes the three-leg vertices $\tilde{\Lambda}_{\alpha \beta \mu}$ (see main text for details).

The diagrammatic representation of Eq. (59) is shown in Fig. 2(a), where the solid line corresponds to the Nambu propagator, the gray circle corresponds to $\overline{\mathbf{h}}_{s}$, and the gray rectangle corresponds to the interaction vertex $\tilde{\Gamma}_{\alpha \beta \gamma \delta}^{s}\left(\mathbf{k}, \mathbf{k}^{\prime}, \mathbf{q}\right)$. The diagrammatic representation for the interaction vertex $\tilde{\Gamma}_{\alpha \beta \gamma \delta}^{s}\left(\mathbf{k}, \mathbf{k}^{\prime}, \mathbf{q}\right)$ is shown in Fig. 2(b), where the solid circles correspond to the three-leg vertices $\tilde{\Lambda}_{\alpha \beta \mu}$. The double wavy line corresponds to $\mathcal{M}_{\mu \nu}^{-1}(\mathbf{q})$, which can be viewed as the dressed bosonic propagator (see Appendix F) summing the particle-hole bubbles, for $s \in\left\{\mathrm{ch}, \mathrm{sp}\right.$, orb $\left., \mathrm{so}, \mathrm{orb}^{*}, \mathrm{so}^{*}\right\}$, or the particle-particle bubbles, for $s=P$, to the infinite order.

\section{B. Landau Fermi-liquid parameters}

We can now calculate the Landau Fermi-liquid parameters for the considered three-orbital degenerate model from Eq. (64). For each channel $s \in\{\mathrm{ch}, \mathrm{sp}$, orb, so, orb*, so*\}, we have

$$
\begin{aligned}
\Gamma^{s}\left(\mathbf{k}, \mathbf{k}^{\prime}, \mathbf{q}\right)= & -\frac{1}{2 Z^{2}}\left[Z\left(\epsilon_{\mathbf{k}}+\epsilon_{\mathbf{k}+\mathbf{q}}\right)\left(\epsilon_{\mathbf{k}^{\prime}}+\epsilon_{\mathbf{k}^{\prime}+\mathbf{q}}\right) \mathcal{M}_{r_{s} r_{s}}^{-1}(\mathbf{q})\right. \\
& +R_{0}\left(\epsilon_{\mathbf{k}}+\epsilon_{\mathbf{k}+\mathbf{q}}\right) \mathcal{M}_{r_{s} l_{s}}^{-1}(\mathbf{q})+R_{0}\left(\epsilon_{\mathbf{k}^{\prime}}+\epsilon_{\mathbf{k}^{\prime}+\mathbf{q}}\right) \\
& \left.\times \mathcal{M}_{r_{s} l_{s}}^{-1}(\mathbf{q})+\mathcal{M}_{l_{s} l_{s}}^{-1}(\mathbf{q})\right],
\end{aligned}
$$

where we applied $\mathbf{R}=R_{0} I$ and $Z=R_{0}^{2}$ for the degenerate model considered here. The scattering amplitude for each particle-hole channel $s$ can be evaluated from

$$
A_{s}(\mathbf{q})=N_{F} Z^{2}\left\langle\left\langle\Gamma^{s}\left(\mathbf{k}, \mathbf{k}^{\prime}, \mathbf{q}\right)\right\rangle_{\mathbf{k}_{F}}\right\rangle_{\mathbf{k}_{F}^{\prime}}
$$

where we introduce the Fermi surface average

$$
\left\langle\left\langle\Gamma\left(\mathbf{k}, \mathbf{k}^{\prime}\right)\right\rangle_{\mathbf{k}_{F}}\right\rangle_{\mathbf{k}_{F}^{\prime}}=\frac{\sum_{\mathbf{k} k^{\prime}} \Gamma\left(\mathbf{k}, \mathbf{k}^{\prime}\right) \delta_{\mathbf{k}, \mathbf{k}_{F}} \delta_{\mathbf{k}^{\prime}, \mathbf{k}_{F}}}{\sum_{\mathbf{k} \mathbf{k}^{\prime}} \delta_{\mathbf{k}, \mathbf{k}_{F}} \delta_{\mathbf{k}^{\prime}, \mathbf{k}_{F}}} .
$$

Here, $N_{F} \equiv \chi_{\mathcal{O}_{s} \mathcal{O}_{s}}^{(0)}(0)$ is the density of state at the Fermi level, which coincides with the bare susceptibility $\chi_{\mathcal{O}_{s} \mathcal{O}_{s}}^{(0)}$. The Fermi-liquid parameters $F_{s}$ can be extracted from the scattering amplitude (see Appendix G)

$$
A_{s}(\mathbf{q})=\frac{F_{s}(\mathbf{q})}{1+F_{s}(\mathbf{q})}
$$

From the definition of the quasiparticle susceptibility, Eqs. (59) and (68), we obtain the random phase approximation (RPA)-like expression for the susceptibilities

$$
\chi_{\mathcal{O}_{s} \mathcal{O}_{s}}(\mathbf{q})=\frac{\chi_{\mathcal{O}_{s} \mathcal{O}_{s}}^{(0)}(\mathbf{q})}{1+F_{s}(\mathbf{q})},
$$

for $s \in\{\mathrm{ch}, \mathrm{sp}$, orb, so, orb*, so* $\}$. Note that we have applied the Fermi-surface average over $\mathbf{k}$ and $\mathbf{k}^{\prime}$. The divergence of the quasiparticle susceptibilities and the scattering amplitudes can be determined from the condition $F_{s}(\mathbf{q})=-1$. Although Eq. (69) has a RPA-like form, the Fermi-liquid parameters are renormalized by the correlation effect for different $\mathbf{q}$, which provides a more accurate description for strongly correlated systems.

\section{Pairing interaction from the particle-particle channel}

The reducible pairing vertex in the orbital-antisymmetric spin-triplet pairing channel $s=\mathrm{P}$ can be computed by projecting the particle-particle scattering vertex $\tilde{\Gamma}^{\mathrm{P}}$ [Eq. (64)] onto the orbital-antisymmetric spin-triplet pairing basis $\mathbf{h}_{P}$ (see Appendix C 2): (a)

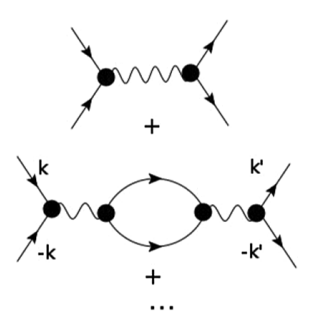

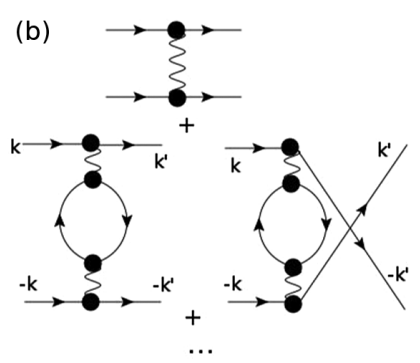

FIG. 3. (a) Pairing vertex from the local particle-particle fluctuation [Eq. (70)]. (b) Pairing vertex from the particle-hole fluctuations [Eq. (74)]. The bubbles are summed to the infinite order. The solid line with arrows corresponds to the normal fermionic propagator. The wavy line corresponds to the bare bosonic propagator. 


$$
\begin{aligned}
\Gamma_{\mathrm{pp}}^{\mathrm{sc}}\left(\mathbf{k}, \mathbf{k}^{\prime}, \mathbf{q}=0\right)= & {\left[\mathbf{h}_{\mathrm{P}}^{\dagger}\right]_{\alpha \beta} \tilde{\Gamma}_{\alpha \beta \gamma \delta}^{P}\left(\mathbf{k}, \mathbf{k}^{\prime}, \mathbf{q}=0\right)\left[\mathbf{h}_{\mathrm{P}}^{\dagger}\right]_{\delta \gamma} } \\
= & -\frac{1}{2 Z^{4}}\left[Z\left(\epsilon_{\mathbf{k}}+\epsilon_{\mathbf{k}}\right)\left(\epsilon_{\mathbf{k}^{\prime}}+\epsilon_{\mathbf{k}^{\prime}}\right) \mathcal{M}_{r_{P} r_{P}}^{-1}(0)\right. \\
& -R_{0}\left(\epsilon_{\mathbf{k}}+\epsilon_{\mathbf{k}}\right) \mathcal{M}_{r_{P} l_{P}}^{-1}(0)-R_{0}\left(\epsilon_{\mathbf{k}^{\prime}}+\epsilon_{\mathbf{k}^{\prime}}\right) \\
& \left.\times \mathcal{M}_{r_{P} l_{P}}^{-1}(0)+\mathcal{M}_{l_{P} l_{P}}^{-1}(0)\right],
\end{aligned}
$$

where we applied $\mathbf{R}=R_{0} I$ and $Z=R_{0}^{2}$ for the degenerate model considered here and restrict the pairing at $\mathbf{q}=0$. The diagrammatic representation for Eq. (70) is shown in Fig. 3(a). In this scattering process, only the particle-particle fermionic bubbles and the local multiplets fluctuation between different particle-number sectors in $\mathcal{M}_{\mu \nu}^{-1}$ are involved [the fluctuation bases $\mathbf{h}_{\mathrm{P}}$ and $\tilde{\mathbf{h}}_{\mathrm{P}}$ in Eqs. (E19)(E26) select the fluctuation that does not conserve the particle number].

We can now derive the RPA-like form for the quasiparticle susceptibility. From $\Gamma_{\mathrm{pp}}^{\mathrm{sc}}$, we compute the reducible pairing interaction by averaging the $\mathbf{k}$ and $\mathbf{k}^{\prime}$ over the Fermi surface

$$
\Gamma_{\mathrm{pp}}^{\mathrm{sc}}=Z^{2}\left\langle\left\langle\Gamma_{\mathrm{pp}}^{\mathrm{sc}}\left(\mathbf{k}, \mathbf{k}^{\prime}\right)\right\rangle_{\mathbf{k}_{F}}\right\rangle_{\mathbf{k}_{F}^{\prime}} .
$$

The irreducible pairing interaction $\Gamma_{\mathrm{pp}}^{\mathrm{irr}}$ can be extracted from (see Appendix G)

$$
\Gamma_{\mathrm{pp}}^{\mathrm{sc}}=\frac{\Gamma_{\mathrm{pp}}^{\mathrm{irr}}}{1+\Gamma_{\mathrm{pp}}^{\mathrm{irr}} \chi_{\mathcal{O}_{P} \mathcal{O}_{P}}^{(0)}} .
$$

From the definition of the quasiparticle susceptibility, Eqs. (59) and (72), we obtain the RPA-like expression for the pairing susceptibility,

$$
\chi_{P}=\frac{\chi_{\mathcal{O}_{P} \mathcal{O}_{P}}^{(0)}}{1+\Gamma_{\mathrm{pp}}^{\mathrm{irr}} \chi_{\mathcal{O}_{P} \mathcal{O}_{P}}^{(0)}}
$$

The divergence of the pairing susceptibilities and vertex can be determined from the condition $\Gamma_{\mathrm{pp}}^{\mathrm{irr}} \chi_{\mathcal{O}_{P} \mathcal{O}_{P}}^{(0)}=-1$.

\section{Pairing interaction from the particle-hole channel}

Besides the $s$-wave pairing induced from the particleparticle vertex, the particle-hole vertices can also induce the local and the nonlocal pairing through the charge and spinfluctuation mechanism [63-66]. To compute the irreducible pairing vertex for the orbital-antisymmetric spin-triplet pairing, we again project the particle-hole vertices onto the pairing basis $\mathbf{h}_{P}$ :

$$
\begin{aligned}
& \left.\Gamma_{\mathrm{ph}}^{\mathrm{irr}}\left(\mathbf{k}, \mathbf{k}^{\prime}\right)=\sum_{\substack{s \in\{\text { ch.sp.orb, } \\
\text { so,orb }}}\left[\mathbf{h}_{\mathrm{P}}^{\dagger}\right]_{\alpha \gamma}^{\dagger}\right\} \tilde{\Gamma}_{\alpha \beta \gamma \delta}^{s}\left(\mathbf{k}, \mathbf{k}^{\prime}\right)\left[\mathbf{h}_{\mathrm{P}}^{\dagger}\right]_{\beta \delta} \\
& =\frac{1}{8}\left[\Gamma^{\mathrm{ch}}\left(\mathbf{k}, \mathbf{k}^{\prime}, \mathbf{q}=\mathbf{k}-\mathbf{k}^{\prime}\right)+\Gamma^{\mathrm{sp}}\left(\mathbf{k}, \mathbf{k}^{\prime}, \mathbf{q}=\mathbf{k}-\mathbf{k}^{\prime}\right)-\Gamma^{\mathrm{orb}}\left(\mathbf{k}, \mathbf{k}^{\prime}, \mathbf{q}=\mathbf{k}-\mathbf{k}^{\prime}\right)-\Gamma^{\mathrm{so}}\left(\mathbf{k}, \mathbf{k}^{\prime}, \mathbf{q}=\mathbf{k}-\mathbf{k}^{\prime}\right)\right. \\
& \left.-\frac{5}{3} \Gamma^{\mathrm{orb}^{*}}\left(\mathbf{k}, \mathbf{k}^{\prime}, \mathbf{q}=\mathbf{k}-\mathbf{k}^{\prime}\right)-\frac{5}{3} \Gamma^{\mathrm{so}^{*}}\left(\mathbf{k}, \mathbf{k}^{\prime}, \mathbf{q}=\mathbf{k}-\mathbf{k}^{\prime}\right)+\left(\mathbf{k}^{\prime} \rightarrow-\mathbf{k}^{\prime}\right)\right],
\end{aligned}
$$

where the charge, spin, orbital, and spin-orbital scattering vertices $\tilde{\Gamma}^{s}$ are defined in Eq. (65). The diagrammatic representation for Eq. (74) is shown in Fig. 3(b), where the $\mathcal{M}_{r_{s} r_{s}}^{-1}, \mathcal{M}_{r_{s} l_{s}}^{-1}$, and $\mathcal{M}_{l_{s} l_{s}}^{-1}$ contain the summation of the particle-hole bubbles to the infinite order (see Appendix F), and we include both the direct and the exchange (crossing) diagrams. The irreducible pairing interaction from the particle-hole channel can be computed from

$$
\Gamma_{\mathrm{ph}}^{\mathrm{irr}}=Z^{2}\left\langle\left\langle\Gamma_{\mathrm{ph}}^{\mathrm{irr}}\left(\mathbf{k}, \mathbf{k}^{\prime}\right)\right\rangle_{\mathbf{k}_{F}}\right\rangle_{\mathbf{k}_{F}^{\prime}},
$$

where we assume an $s$-wave pairing to compare with the local pairing fluctuation mechanism in the previous section.

\section{RESULTS AND DISCUSSION}

\section{A. Superconducting phase diagram}

In this subsection, we apply our RISB saddle-point approximation and fluctuation approach to the degenerate three-orbital Hubbard-Kanamori model with Hund's coupling $J=U / 4$, which serves as an effective model for Hund's metals. We focus on the order parameter $\left\langle\hat{\mathcal{O}}_{\mathrm{P}}\right\rangle$ computed from Eq. (19) and the pairing susceptibility $\chi_{\mathrm{P}}$ computed from Eq. (42).

Figure 4(a) shows the intensity plot of the spin-triplet pairing order parameter $\left\langle\hat{\mathcal{O}}_{\mathrm{P}}\right\rangle$ at $T=0.0005 t$. The peak of the order parameters is located at the so-called Hund's metal crossover, where the quasiparticle weights $Z$ decrease significantly, as shown in Figs. 4(c) and 4(d) for selected fillings $n=1.6,2.0,2.4$, and 2.8. The faster the decrease in $Z$, the stronger the enhancement in the pairing order parameters $\left\langle\hat{\mathcal{O}}_{\mathrm{P}}\right\rangle$. The normal state in the superconducting regime can be viewed as Hund's metals, where the quasiparticle weight is small, and the local multiplet is populated with high spin states, favoring the local spintriplet pairing [16,53,67-69].

We also show the uniform pairing susceptibility $\chi_{\mathrm{P}}$ evaluated from the fluctuation technique in Fig. 4(b). 

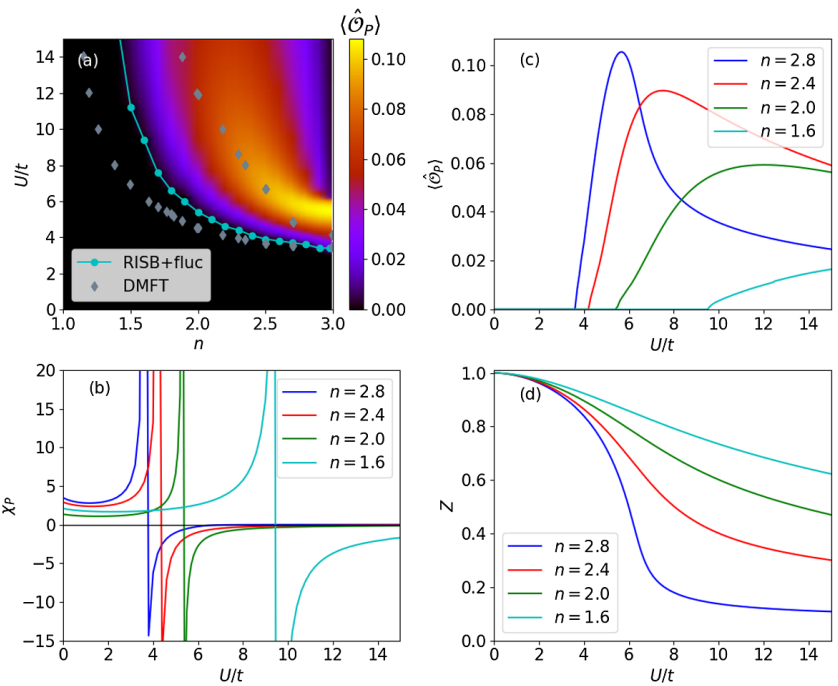

FIG. 4. (a) Density plot of the $s$-wave spin-triplet superconducting order parameter $\left\langle\hat{\mathcal{O}}_{P}\right\rangle$ as a function of electron filling $n$ and Coulomb interaction $U$ with $J=U / 4$ at $T=0.0005 t$. The cyan line is the phase boundary determined from the instability in the pairing susceptibility $\chi_{\mathrm{P}}$. (b) Uniform pairing susceptibility $\chi_{\mathrm{P}}$ for $n=2.8,2.4,2.0,1.6$. (c) Spin-triplet superconducting order parameters $\left\langle\hat{\mathcal{O}}_{P}\right\rangle$ for $n=2.8,2.4,2.0,1.6$. (d) Quasiparticle weight $Z$ for $n=2.8,2.4,2.0,1.6$.

The pairing susceptibility is initially positive at small Coulomb interaction $U$ and diverges at the critical point. Then, the pairing susceptibility becomes negative, indicating the instability towards the $s$-wave spin-triplet ordering state. The phase boundary determined from the divergence of the pairing susceptibility is shown in Fig. 4(a), which agrees with the onset of the mean-field order parameters indicating the consistency of our approach. We also compare our phase diagram with the DMFT results on a Bethe lattice at $T=0.04 t$ rescaled to the 2D bandwidth $W=8 t$ in Fig. 4(a). While the RISB superconducting regime is broader than the DMFT results, the overall phase diagram agrees qualitatively with the DMFT [53].

We now turn to the finite-temperature phase diagram for the $s$-wave spin-triplet pairing state. Figure 5(a) shows the intensity plot of the $s$-wave spin-triplet order parameters $\left\langle\hat{\mathcal{O}}_{\mathrm{P}}\right\rangle$ at $U=8 t$ as a function of electron filling $n$ and temperature $T$. The superconducting region has a domeshape structure, where the maximum $T_{c}$ is located around $n=2.5$. Figure 5 (b) shows the uniform pairing susceptibility $\chi_{\mathrm{P}}$ computed from the fluctuation approach for filling $n=2.0,2.4$, and 2.8 as a function of temperature $T$. With decreasing $T$, the pairing susceptibility increases and diverges at the critical temperature $T_{c}$. The critical temperature obtained from the divergence of the pairing susceptibility agrees with the onset of the mean-filed order parameters, as shown in Fig. 5(a). We also compare our phase diagram with the DMFT results on a Bethe lattice in Fig. 5(a) corresponding to $U=6 t$ rescaled to the 2D
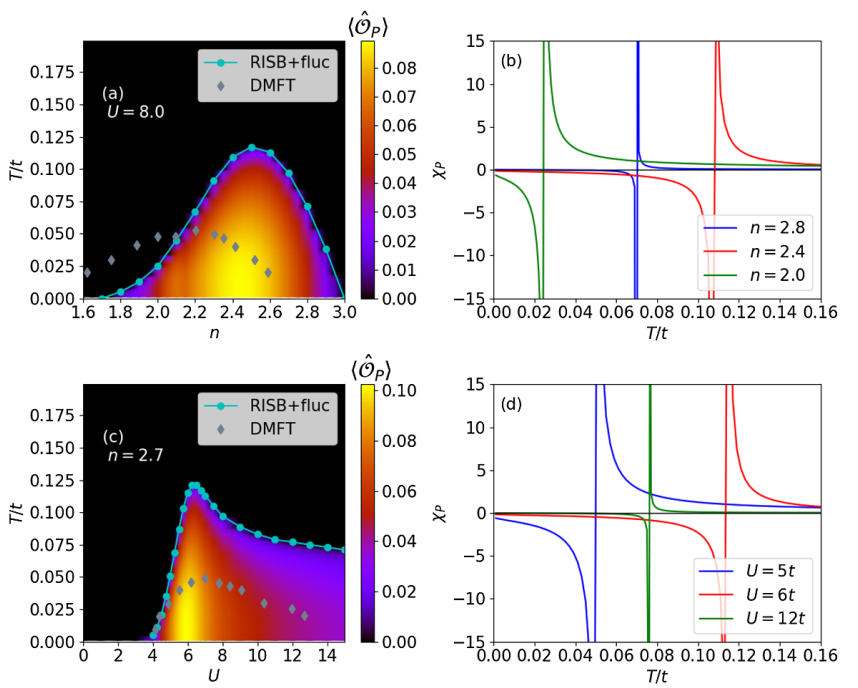

FIG. 5. (a) Density plot of the $s$-wave spin-triplet superconducting order parameter $\left\langle\hat{\mathcal{O}}_{P}\right\rangle$ as a function of electron filling $n$ and temperature $T$ at $U=8$ and $J=U / 4$. The cyan line is the phase boundary determined from the instability of the pairing susceptibility $\chi_{\mathrm{P}}$. (b) Uniform pairing susceptibility $\chi_{\mathrm{P}}$ for $n=2.8,2.4,2.0$. (c) Density plot of the spin-triplet superconducting order parameter $\left\langle\hat{\mathcal{O}}_{P}\right\rangle$ as a function of Coulomb interaction $U$ and temperature $T$ with $n=2.7$ and $J=U / 4$. The cyan line is the phase boundary determined from the instability of the pairing susceptibility $\chi_{\mathrm{P}}$. (d) Uniform pairing susceptibility $\chi_{\mathrm{P}}$ for $U=5 t, 16 t, 12 t$.

bandwidth $W=8 t$ considered here [53]. Both methods generate a dome-shape structure where the peak in RISB is closer to half-filling.

Figure 5(c) shows the intensity plot of the $s$-wave spintriplet pairing order parameters $\left\langle\hat{\mathcal{O}}_{\mathrm{P}}\right\rangle$ as a function of Coulomb interaction $U$ and $J=U / 4$ at filling $n=2.7$. The critical temperature $T_{c}$ peaks around $U=6 t$, which is around Hund's metal crossover. Figure 5(d) shows the corresponding uniform pairing susceptibility $\chi_{\mathrm{P}}$ computed from the fluctuation approach for $U=5 t, 6 t$, and $12 t$. The pairing susceptibility diverges at $T_{c}$ and becomes negative, indicating the instability towards the $s$-wave spin-triplet pairing states. The $T_{c}$ obtained from the divergence of the susceptibility again agrees with the onset of the mean-field order parameters, as shown in Fig. 5(c). We also compare our phase diagram with the DMFT results on a Bethe lattice in Fig. 5(c) at $n=2.0$ to match with our critical $U_{c}$ at $T=0.0005 t$. The phase diagrams obtained from both methods are again similar, with a dome-shape structure where the $T_{c}$ peaks around Hund's crossover.

Note that there are two main reasons for expecting qualitative agreement (but quantitative agreement) between our RISB results and the DMFT results of Ref. [53]. The first reason is that RISB (equivalently, GA) is essentially a variational approximation to DMFT, in the sense that it is variational in the limit of infinite dimension [70], where 
DMFT is exact. Also, RISB can be viewed as an approximation to DMFT, from a quantum embedding perspective, where the uncorrelated bath has the same number of orbitals as the impurity (while the bath is infinite in DMFT). Hence, RISB is expected to be less accurate (but more efficient) compared to DMFT. Nevertheless, we note that, in this work, we assume a 2D square lattice, while a Bethe lattice was used in Ref. [53]. In fact, it is known that different lattice structures can lead to quantitative differences in the results, but the qualitative behaviors are generally similar [71].

\section{B. Landau parameter and pairing interaction}

For studying the pairing mechanism, it is instructive to investigate the quasiparticle interaction vertex in the spin, charge, orbital, spin-orbital, and pairing channels. To obtain these quantities, we applied the Fermi-liquid approximation in Sec. IV, which reproduces the exact physical susceptibility, as shown in Appendix J.

Let us first discuss the charge, spin, orbital, and spinorbital fluctuations, encoded in the Landau parameters $F_{s}$. The Landau parameters $F_{s}$ in each channel are shown in Fig. 6. We found that the Landau parameters in the charge $F_{\text {ch }}$ and orbital $F_{\text {orb(orb*) }}$ channels show a peak around Hund's crossover and diverge at the Mott transition at $n=3$. The kink in $F_{\text {ch }}$ corresponds to the possible phase separation instability found in the previous slave-spin study [72]. Moreover, we found the instability towards the
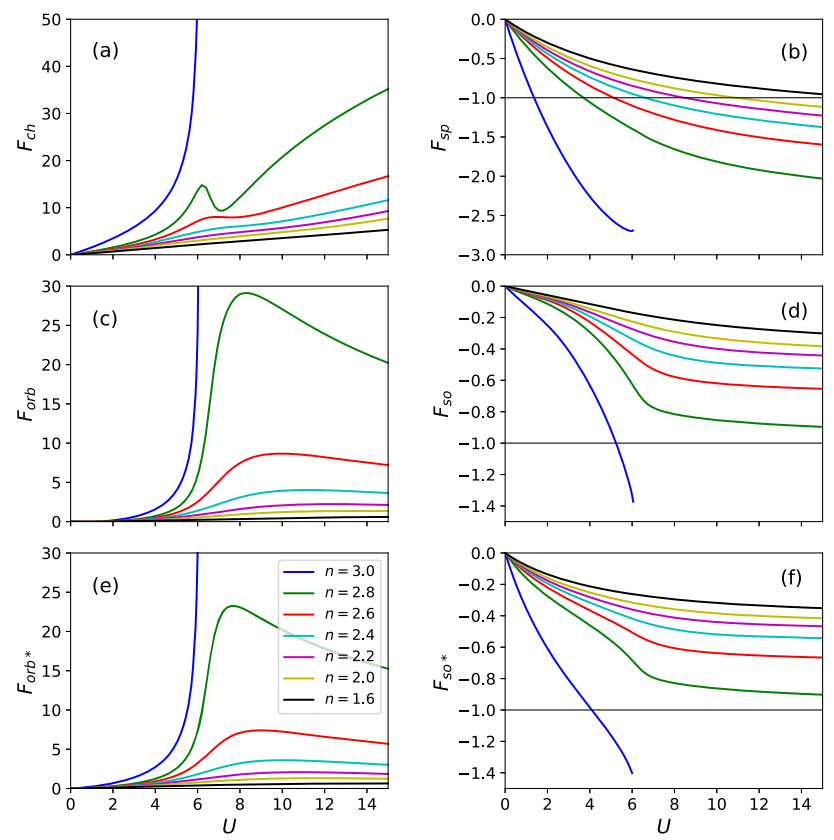

FIG. 6. The Landau parameters in the (a) charge, (b) spin, (c) orbital, (d), spin-orbital, (e) orbital*, and (f) spin-orbital* channel defined in Eq. (5) as a function of coulomb interaction $U$ and $J=U / 4$ for filling $n=3.0,2.8,2.6,2.4,2.2,2.0,1.6$ and $T=0.0005 t$. ferromagnetic ordering $F_{\mathrm{sp}}=-1$ for a wide range of electron filling. Consequently, $F_{\mathrm{sp}}$ is the dominant fluctuation in the particle-hole channel. In addition, the spinorbital channel $F_{\text {so(so*) }}$ also shows a subleading instability at $n=3$.

We now turn to the irreducible pairing vertex in the particle-particle channel $\Gamma_{\mathrm{pp}}^{\mathrm{irr}}$ originating purely from the local pairing fluctuation describing the superconducting instability. Figure 7(a) shows the behavior of the pairing interaction $\Gamma_{\mathrm{pp}}^{\mathrm{irr}}$ in the particle-particle channel as a function of Coulomb interaction $U$. The condition $\Gamma_{\mathrm{pp}}^{\mathrm{irr}} \chi_{O_{P}}^{(0)}=-1$ indicates the divergence in the pairing susceptibility. In the weak-coupling limit, i.e., $U \ll t, \Gamma_{\mathrm{pp}}^{\mathrm{irr}}$ follows the bare pairing interaction $U-3 J$ for all the electron filling $n$. With increasing $U$, the effective interactions for different electronic filling are renormalized to smaller values and eventually become negative, signalizing the instability towards the pairing states. The pairing instability determined from $\Gamma_{\mathrm{pp}}^{\mathrm{irr}}$ is located around Hund's metal crossover as discussed in the previous subsection. On the other hand, as shown in Fig. 7(b), the pairing instability determined from the particle-hole scattering channel $\Gamma_{\mathrm{ph}}^{\mathrm{irr}}$ takes place at a much lower $U$ below Hund's metal crossover. Consequently, the particle-hole spin-fluctuation mechanism cannot explain the pairing instability around Hund's metal crossover. The strong attraction in $\Gamma_{\mathrm{ph}}^{\mathrm{irr}}$ is, however, related to the ferromagnetic instability, as shown in Fig. 6(b).

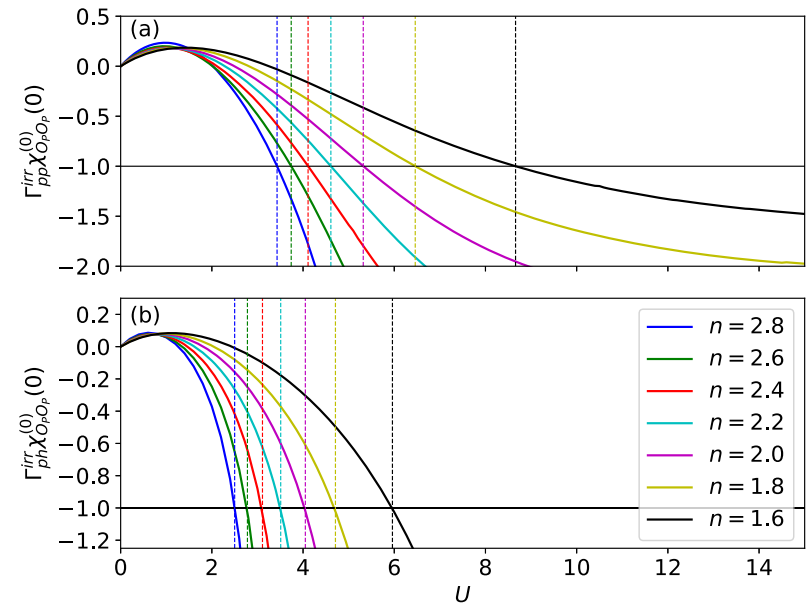

FIG. 7. (a) Irreducible particle-particle $s$-wave spin-triplet pairing vertex $\Gamma_{\mathrm{pp}}^{\mathrm{irr}} \chi_{O_{P}}^{(0)}$ as a function of Coulomb interaction $U$ and $J=U / 4$ for filling $n=2.8,2.6,2.4,2.2,2.0,1.8,1.6$ and temperature $T=0.0005 t$. (b) Irreducible particle-hole $s$-wave spin-triplet pairing vertex $\Gamma_{\mathrm{ph}}^{\mathrm{irr}} \chi_{O_{P}}^{(0)}$ with the same parameter settings. The vertical dashed lines indicate the critical $U_{c}$ determined from $\Gamma_{\mathrm{pp}}^{\mathrm{irr}} \chi_{0}^{P}=-1$, signalizing the divergence of the superconducting susceptibility and the scattering amplitude. 


\section{CONCLUSIONS}

On the basis of the fluctuation approach around the RISB normal-state saddle point, we developed an efficient method to compute general susceptibilities, the quasiparticle interaction vertex, Fermi-liquid parameters, and pairing interaction for the multiorbital Hubbard model. The method has a RPA-like efficiency and a similar accuracy compared to DMFT for correlated systems.

We applied our method to the degenerate three-orbital Hubbard-Kanamori model to investigate the origin of the $s$-wave orbital-antisymmetric spin-triplet pairing in Hund's metal, previously found in the DMFT studies [53]. We showed that, in agreement with DMFT, the pairing susceptibility of the $s$-wave spin-triplet pairing states diverges around Hund's metal crossover. The phase diagram is in good qualitative agreement with DMFT. By computing the pairing interaction by considering the particle-particle and the particle-hole scattering channels, we identified that the origin of the superconducting pairing around Hund's crossover arises from the particle-particle channel, containing the local electron pair fluctuation between different particlenumber sectors of the local Hilbert space. The pairing interaction is strongly renormalized in the incoherent Hund's metal regime and becomes negative. On the other hand, the particle-hole spin-fluctuation mechanism induces an $s$-wave pairing instability already for a smaller value of Coulomb interaction before entering Hund's regime.

The local interorbital pairing mechanisms revealed in this work can be applied to the $s$-wave orbital-antisymmetric spin-triplet pairing states proposed for $\mathrm{Sr}_{2} \mathrm{RuO}_{4}[59,60,73-$ 75] and $\mathrm{KFe}_{2} \mathrm{As}_{2}$ [76,77], where the interplay between Hund's rule coupling and the spin-orbital coupling leads to intriguing gap structures on the Fermi surface. Our approach provides an efficient route for investigating the pairing mechanism for these materials, with the combination of density functional theory. The general formalism that we presented is also applicable for different purposes. For example, it could be utilized for investigating the response functions in the correlation-induced topological materials, e.g., the topological Kondo and topological Mott insulators $[22,78-80]$, and the recently proposed topological iron-based superconductors [81,82]. In addition, the diagrammatic approach proposed in this work may serve as a basis for the nonlocal extensions beyond RISB, similarly to the diagrammatic approaches beyond DMFT [83]. Finally, our formalism can be applied to the NIB-DMET and other similar quantum embedding methods [84-86].

\section{ACKNOWLEDGMENTS}

This work was supported by the Computational Materials Sciences Program funded by the U.S. Department of Energy, Office of Science, Basic Energy Sciences, Materials Sciences and Engineering division. N. L. gratefully acknowledges support from the Novo Nordisk Foundation through the Exploratory Interdisciplinary Synergy Programme Project No. NNF19OC0057790, and from the VILLUM FONDEN through the Villum Experiment Project No. 00028019 and the Centre of Excellence for Dirac Materials (Grant No. 11744).

\section{APPENDIX A: GELL-MANN MATRICES}

We use the following convention for the Gell-Mann matrices:

$$
\begin{aligned}
\lambda^{1}=\left(\begin{array}{lll}
0 & 1 & 0 \\
1 & 0 & 0 \\
0 & 0 & 0
\end{array}\right), & \lambda^{4}=\left(\begin{array}{ccc}
0 & -i & 0 \\
i & 0 & 0 \\
0 & 0 & 0
\end{array}\right), & \lambda^{7}=\left(\begin{array}{ccc}
1 & 0 & 0 \\
0 & -1 & 0 \\
0 & 0 & 0
\end{array}\right), \\
\lambda^{2}=\left(\begin{array}{lll}
0 & 0 & 1 \\
0 & 0 & 0 \\
1 & 0 & 0
\end{array}\right), & \lambda^{5}=\left(\begin{array}{ccc}
0 & 0 & -i \\
0 & 0 & 0 \\
i & 0 & 0
\end{array}\right), & \lambda^{8}=\frac{1}{\sqrt{3}}\left(\begin{array}{ccc}
1 & 0 & 0 \\
0 & 1 & 0 \\
0 & 0 & -2
\end{array}\right), \\
\lambda^{3}=\left(\begin{array}{ccc}
0 & 0 & 0 \\
0 & 0 & 1 \\
0 & 1 & 0
\end{array}\right), & \lambda^{6}=\left(\begin{array}{ccc}
1 & 0 & 0 \\
0 & 0 & -i \\
0 & i & 0
\end{array}\right), & \lambda^{0}=\left(\begin{array}{lll}
0 & 1 & 0 \\
0 & 0 & 1
\end{array}\right),
\end{aligned}
$$

where $\lambda^{1}, \lambda^{2}, \lambda^{3}$ describe the symmetric interorbital interactions or pairings; $\lambda^{4}, \lambda^{5}, \lambda^{6}$ describe the antisymmetric interorbital interactions or pairings; and $\lambda^{7}, \lambda^{8}, \lambda^{0}$ describe the intraorbital interactions or pairings. This set of matrices is the most general basis that parametrizes the $3 \times 3$ quadratic operators in the orbital space for three-orbital models. In the degenerate three-orbital Hubbard-Kanamori model, the $O(3)$ symmetry implies that the order parameters corresponding to the symmetric interorbital fluctuations $\lambda_{1}, \lambda_{2}$, and $\lambda_{3}$ are identical to each other. Similarly, the order parameters corresponding to the antisymmetric interorbital fluctuations $\lambda_{4}, \lambda_{5}$, and $\lambda_{6}$ are identical to each other. 


\section{APPENDIX B: ROTATIONALLY INVARIANT SLAVE-BOSON NAMBU FORMALISM}

In this section, we outline the basis of the RISB Nambu formalism. We start from a generic multiorbital Hubbard model in the Nambu notation:

$$
\hat{H}=\frac{1}{2} \sum_{\mathbf{k}} \Xi_{\mathbf{k} \alpha}^{\dagger} \tilde{\epsilon}_{\mathbf{k}, \alpha \beta} \Xi_{\mathbf{k} \beta}+\sum_{i} \hat{H}_{\mathrm{loc}}\left[\left\{d_{i \alpha \sigma}^{\dagger}, d_{i \alpha \sigma}\right\}\right],
$$

where

$$
\tilde{\mathbf{\epsilon}}_{\mathbf{k}, \alpha \beta}=\left(\begin{array}{cc}
\epsilon_{\mathbf{k}} & 0 \\
0 & -\epsilon_{-\mathbf{k}}^{*}
\end{array}\right)
$$

is the energy dispersion in the Nambu basis. We also define the Nambu spinor $\Xi_{\mathbf{k}}^{\dagger}=\left(d_{\mathbf{k} 1 \uparrow}^{\dagger} d_{\mathbf{k} 1 \downarrow}^{\dagger} \cdots\right.$ $\left.d_{\mathbf{k} M \uparrow}^{\dagger} d_{\mathbf{k} M \downarrow}^{\dagger} d_{-\mathbf{k} 1 \uparrow} d_{-\mathbf{k} 1 \downarrow} \ldots d_{-\mathbf{k} M \uparrow} d_{-\mathbf{k} M \downarrow}\right)$, where $M$ is the total number of orbitals. The $H_{\text {loc }}$ contains the generic local one-body and two-body interactions.

Within the RISB framework, the physical operator $\Xi_{i \alpha}$ is mapped to the product of a renormalization matrix and a quasiparticle Nambu spinor:

$$
\Xi_{i \alpha}^{\dagger}=\sum_{a} \mathbf{R}_{i a \alpha}\left[\Phi_{i}^{\dagger}, \Phi_{i}\right] \Psi_{i a}^{\dagger},
$$

where the quasiparticle spinor is $\Psi_{i}^{\dagger}=\left(f_{i 1 \uparrow}^{\dagger} f_{i 1 \downarrow}^{\dagger} \ldots\right.$, $\left.f_{i M \uparrow}^{\dagger} f_{i M \downarrow}^{\dagger}, f_{i 1 \uparrow} f_{i 1 \downarrow} \ldots f_{i M \uparrow} f_{i M \downarrow}\right)$, and the renormalization matrix has the following form $[62,87,88]$ :

$$
\mathbf{R}_{i a \alpha}\left[\Phi_{i}^{\dagger}, \Phi_{i}\right]=\sum_{b} \operatorname{Tr}\left[\Phi_{i}^{\dagger} \Xi_{i \alpha} \Phi_{i} \Psi_{i b}^{\dagger}\right]\left[\boldsymbol{\Delta}_{i}\left(1-\boldsymbol{\Delta}_{i}\right)\right]_{b a}^{-\frac{1}{2}},
$$

where

$$
\left[\boldsymbol{\Delta}_{i}\right]_{a b}=\operatorname{Tr}\left[\Phi_{i}^{\dagger} \Phi_{i} \Psi_{i a}^{\dagger} \Psi_{i b}\right]
$$

corresponds to the local quasiparticle Nambu density matrix, and $\left[\Phi_{i}\right]_{A n}$ is the slave-boson amplitude matrix. We also define the matrices $\left[\Xi_{i \alpha}\right]_{A B}=\left\langle A\left|\hat{\Xi}_{i \alpha}\right| B\right\rangle$ and $\left[\Psi_{i \alpha}\right]_{n m}=\left\langle n\left|\hat{\Psi}_{i \alpha}\right| m\right\rangle$ for the fermionic operator in the arbitrary, local many-body basis $|A\rangle$ and the local Fock basis $|n\rangle$, respectively $[9,21]$. The local interactions can be expressed in terms of the bosonic amplitudes as [9]

$$
\hat{H}_{\mathrm{loc}}=\sum_{A B n}[\Phi]_{B n}[\Phi]_{n A}^{\dagger}\left[H_{\mathrm{loc}}\right]_{A B},
$$

where $\left[H_{\mathrm{loc}}\right]_{A B}=\left\langle A\left|\hat{H}_{\mathrm{loc}}\right| B\right\rangle$.

In order to select the physical states out of the enlarged boson and quasiparticle Hilbert space, one has to enforce the following RISB constraints $[9,62]$ :

$$
\operatorname{Tr}\left[\Phi_{i} \Phi_{i}^{\dagger}\right]=1
$$

$$
\left[\boldsymbol{\Delta}_{i}\right]_{a b}=\left\langle\hat{\Psi}_{i a}^{\dagger} \hat{\Psi}_{i b}\right\rangle=\operatorname{Tr}\left[\Phi_{i}^{\dagger} \Phi_{i} \Psi_{i a}^{\dagger} \Psi_{i b}\right]
$$

The first constraint limits the Hilbert space to the singleboson states, while the second constraint ensures the rotational invariance of the quasiparticle density matrix under the gauge transformation (see Appendix $\mathrm{H}$ ).

With the RISB representations and constraints [Eqs. (B3)-(B8)], the RISB Lagrangian for the generic Hubbard model [Eq. (B1)] can be expressed as

$$
\begin{aligned}
\mathcal{L}\left[\Phi, \mathbf{R}, \boldsymbol{\Lambda} ; \mathbf{D}, \boldsymbol{\Lambda}^{c}, E^{c}, \Delta\right]= & \frac{-T}{N} \frac{1}{2} \sum_{\mathbf{k}_{1} \mathbf{k}_{2} \omega_{n}} \operatorname{Tr} \log \left[-i \omega_{n}+H_{\mathbf{k}_{1} \mathbf{k}_{2}}^{\mathrm{qp}}\right] e^{i \omega_{n} 0^{+}}+\sum_{i} \operatorname{Tr}\left[\Phi_{i} \Phi_{i}^{\dagger} H_{\mathrm{loc}}\right. \\
& \left.+\left(\sum_{a \alpha}\left[\mathbf{D}_{i}\right]_{a \alpha} \Phi_{i}^{\dagger} \Xi_{i \alpha}^{\dagger} \Phi_{i} \Psi_{i a}+\text { H.c. }\right)+\sum_{a b} \frac{1}{2}\left[\boldsymbol{\Lambda}_{i}^{c}\right]_{a b} \Phi_{i}^{\dagger} \Phi_{i} \Psi_{i a}^{\dagger} \Psi_{i b}\right]+E_{i}^{c}\left(\operatorname{Tr}\left[\Phi_{i} \Phi_{i}^{\dagger}\right]-1\right) \\
& -\sum_{i}\left[\sum_{a b} \frac{1}{2}\left(\left[\boldsymbol{\Lambda}_{i}\right]_{a b}+\left[\boldsymbol{\Lambda}_{i}^{c}\right]_{a b}\right)\left[\boldsymbol{\Delta}_{i}\right]_{a b}+\sum_{c a \alpha}\left(\left[\mathbf{D}_{i}\right]_{a \alpha}\left[\mathbf{R}_{i}\right]_{c \alpha}\left[\boldsymbol{\Delta}_{i}\left(1-\boldsymbol{\Delta}_{i}\right)\right]_{c a}^{1 / 2}+\text { c.c. }\right)\right],
\end{aligned}
$$

where the original kinetic hopping term in Eq. (B1) is described by the quasiparticle Hamiltonian:

$\left[H_{\mathbf{k}_{1} \mathbf{k}_{2}}^{\mathrm{qp}}\right]_{a b}=\frac{1}{N} \sum_{\mathbf{k}}\left[\mathbf{R}_{\mathbf{k}_{1}-\mathbf{k}} \tilde{\epsilon}_{\mathbf{k}} \mathbf{R}_{\mathbf{k}_{2}-\mathbf{k}}^{\dagger}\right]_{a b}+\left[\boldsymbol{\Lambda}_{\mathbf{k}_{1}-\mathbf{k}_{2}}\right]_{a b}$,

while the local interaction $\hat{H}_{\text {loc }}$ in Eq. (B1) is mapped to the slave-boson representation $\operatorname{Tr}\left[\Phi_{i} \Phi_{i}^{\dagger} H_{\mathrm{loc}}\right]$. The $\boldsymbol{\Lambda}_{i}, \boldsymbol{\Lambda}_{i}^{c}, \mathbf{D}_{i}$, and $E_{i}^{c}$ are the Lagrange multipliers enforcing the RISB constraints [Eqs. (B7) and (B8)] and the structure of the $\mathbf{R}_{i}$ matrix [Eq. (B4)]. Note that all these single-particle matrices contain the particle, hole, and anomalous sectors defined as follows:

$$
\mathbf{R}_{i}=\left[\begin{array}{cc}
R_{i} & Q_{i}^{*} \\
Q_{i} & R_{i}^{*}
\end{array}\right]
$$




$$
\begin{gathered}
\boldsymbol{\Lambda}_{i}=\left[\begin{array}{cc}
\Lambda_{i} & \Lambda_{i}^{\prime} \\
\Lambda_{i}^{\prime \dagger} & -\Lambda_{i}^{*}
\end{array}\right], \\
\boldsymbol{\Lambda}_{i}^{c}=\left[\begin{array}{cc}
\Lambda_{i}^{c} & \Lambda_{i}^{c \prime} \\
\Lambda_{i}^{c \prime} & -\Lambda_{i}^{c *}
\end{array}\right], \\
\mathbf{D}_{i}=\left[\begin{array}{cc}
D_{i} & D_{i}^{\prime *} \\
D_{i}^{\prime} & D_{i}^{*}
\end{array}\right], \\
\boldsymbol{\Delta}_{i}=\left[\begin{array}{cc}
\Delta_{i} & \Delta_{i}^{\prime} \\
\Delta_{i}^{\prime \dagger} & \left(\mathbf{1}-\Delta_{i}\right)
\end{array}\right] .
\end{gathered}
$$

The $\boldsymbol{\Lambda}_{i}, \boldsymbol{\Lambda}_{i}^{c}$, and $\boldsymbol{\Delta}_{i}$ are Hermitian matrices, and the $\mathbf{R}_{i}$ and $\mathbf{D}_{i}$ are non-Hermitian matrices. These single-particle matrices are parametrized by Eqs. (21)-(25) utilizing the matrix bases $\mathbf{h}_{s}$ and $\tilde{\mathbf{h}}_{s}$, whose structure (for the threeorbital degenerate Hubbard-Kanamori model) is discussed in Appendix C 2.

The slave-boson amplitude can be constructed from the symmetry adaptive basis $\phi_{i p}$ :

$$
\left[\Phi_{i}\right]_{A n}=\sum_{p} c_{p}\left[\phi_{i p}\right]_{A n}
$$

where

$$
\operatorname{Tr}\left[\phi_{i p}^{\dagger} \phi_{i p^{\prime}}\right]=\delta_{p, p^{\prime}} \quad p, p^{\prime}=1, \ldots, N_{\phi},
$$

and the matrix basis commutes with the symmetry operation in the group $G$ of the given problem, i.e., $\left[\phi_{i p}, R(g)\right]=0 \forall g \in G$. The procedure for determining $\phi_{i p}$ is discussed in Appendix C 1.

\section{Embedding mapping}

We now introduce the embedding wave function [21]

$$
\left|\Phi_{i}\right\rangle=\sum_{A n} e^{i(\pi / 2) N_{n}\left(N_{n}-1\right)}\left[\Phi_{i}\right]_{A n} U_{\mathrm{PH}}|A\rangle|n\rangle,
$$

where $U_{\mathrm{PH}}$ is the particle-hole transformation on the bath site and $N_{n}$ is the particle number of Fock state $|n\rangle$. Substituting the following identities using Eq. (B9):

$$
\begin{array}{r}
\operatorname{Tr}\left[\Phi_{i} \Phi_{i}^{\dagger} H_{\mathrm{loc}}\right]=\left\langle\Phi_{i}\left|\hat{H}_{\mathrm{loc}}\left[\hat{d}_{i \alpha}^{\dagger}, \hat{d}_{i \alpha}\right]\right| \Phi_{i}\right\rangle, \\
\operatorname{Tr}\left[\Phi_{i}^{\dagger} \Xi_{i \alpha}^{\dagger} \Phi \Psi_{i a}\right]=\sum_{b}\left\langle\Phi_{i}\left|\hat{\Xi}_{i \alpha}^{\dagger} \hat{\Psi}_{i b}\right| \Phi_{i}\right\rangle \bar{I}_{b a}, \\
\operatorname{Tr}\left[\Phi_{i}^{\dagger} \Phi_{i} \Psi_{i a}^{\dagger} \Psi_{i b}\right]=\sum_{c d} \bar{I}_{b c}\left\langle\Phi_{i}\left|\hat{\Psi}_{i c} \hat{\Psi}_{i d}^{\dagger}\right| \Phi_{i}\right\rangle \bar{I}_{d a},
\end{array}
$$

where

$$
\bar{I}=\left(\begin{array}{cc}
\mathbf{1} & 0 \\
0 & -\mathbf{1}
\end{array}\right)
$$

and $\mathbf{1}$ is the identity matrix, we obtain the RISB Lagrangian in terms of $\left|\Phi_{i}\right\rangle$ in Eq. (6) in the main text.

\section{APPENDIX C: VARIATIONAL BASIS}

In this section, we describe the construction of our variational many-body basis $\phi_{p}$ and the single-particle bases $\mathbf{h}_{s}$ and $\tilde{\mathbf{h}}_{s}$ of our fluctuation approach to the degenerate three-orbital Hubbard-Kanamori model.

\section{Many-body basis}

For the charge, spin, orbital, and spin-orbital fluctuations, we construct the many-body basis in Eq. (B16) using the symmetry-adapted basis. The procedure can be found in Ref. [89]. On the other hand, for the pairing state, we construct the many-body variational basis following the procedure in Ref. [62]. First, since the Hubbard-Kanamori interaction [Eq. (3)] can be written into

$H_{\text {loc }}=(U-3 J) \frac{\hat{N}(\hat{N}-1)}{2}-J\left[2 \hat{\mathbf{S}}^{2}+\frac{1}{2} \hat{\mathbf{L}}^{2}\right]+\frac{5}{2} J \hat{N}$

with

$$
\begin{gathered}
\hat{L}_{\alpha}=\sum_{\beta \gamma \sigma} \hat{d}_{i \beta \sigma}^{\dagger}\left[-i \epsilon_{\alpha \beta \gamma}\right] \hat{d}_{i \gamma \sigma}, \\
\hat{\mathbf{S}}=\frac{1}{2} \sum_{\alpha \sigma \sigma^{\prime}} \hat{d}_{i \alpha \sigma}^{\dagger} \boldsymbol{\sigma}_{\sigma \sigma^{\prime}} \hat{d}_{i \alpha \sigma^{\prime}}, \\
\hat{N}=\sum_{\alpha \sigma} \hat{d}_{\alpha \sigma}^{\dagger} \hat{d}_{\alpha \sigma},
\end{gathered}
$$

the local Hamiltonian is diagonalized in the $\Gamma=(N, L, S)$ basis. The $\sigma$ is a vector of Pauli matrices, and $\epsilon_{\alpha \beta \gamma}$ is the Levi-Civita symbol, which can be expressed in terms of Gell-Mann matrices $\lambda^{4}, \lambda^{5}$, and $\lambda^{6}$. Therefore, the slaveboson amplitude can be significantly reduced to

$$
\begin{aligned}
\Phi_{\Gamma n}= & \langle\Gamma \mid n\rangle \Phi\left(E_{\Gamma}\right)+\sum_{q=1}^{3}\left[\frac{\left\langle n\left|\left(\hat{\mathcal{O}}_{\mathrm{P}}\right)^{q}\right| \Gamma\right\rangle}{\sqrt{\left\langle\Gamma\left|\left(\hat{\mathcal{O}}_{\mathrm{P}}^{\dagger}\right)^{q}\left(\hat{\mathcal{O}}_{\mathrm{P}}\right)^{q}\right| \Gamma\right\rangle}} \Phi\left(E_{\Gamma} ; 2 q\right),\right. \\
& \left.+\frac{\left\langle n\left|\left(\hat{\mathcal{O}}_{\mathrm{P}}^{\dagger}\right)^{q}\right| \Gamma\right\rangle}{\sqrt{\left\langle\Gamma\left|\left(\hat{\mathcal{O}}_{\mathrm{P}}\right)^{q}\left(\hat{\mathcal{O}}_{\mathrm{P}}^{\dagger}\right)^{q}\right| \Gamma\right\rangle}} \Phi\left(E_{\Gamma} ;-2 q\right)\right], \quad \text { (C5) }
\end{aligned}
$$

where $E_{\Gamma}$ and $|\Gamma\rangle$ are the eigenvalue and the eigenstate of Eq. (C1), respectively. Comparing Eq. (C5) to Eq. (B16), we identify that the many-body basis for the normal-state part is 


$$
\phi_{p}=\langle\Gamma \mid n\rangle,
$$

with the corresponding slave boson $c_{p}=\Phi\left(E_{\Gamma}\right)$, and the pairing parts are

$$
\phi_{p}=\frac{\left\langle n\left|\left(\hat{\mathcal{O}}_{\mathrm{P}}\right)^{q}\right| \Gamma\right\rangle}{\sqrt{\left\langle\Gamma\left|\left(\hat{\mathcal{O}}_{\mathrm{P}}^{\dagger}\right)^{q}\left(\hat{\mathcal{O}}_{\mathrm{P}}\right)^{q}\right| \Gamma\right\rangle}}
$$

and

$$
\phi_{p}=\frac{\left\langle n\left|\left(\hat{\mathcal{O}}_{\mathrm{P}}^{\dagger}\right)^{q}\right| \Gamma\right\rangle}{\sqrt{\left\langle\Gamma\left|\left(\hat{\mathcal{O}}_{\mathrm{P}}\right)^{q}\left(\hat{\mathcal{O}}_{\mathrm{P}}^{\dagger}\right)^{q}\right| \Gamma\right\rangle}}
$$

with the corresponding slave-boson amplitudes $c_{p}=$ $\Phi\left(E_{\Gamma} ; 2 q\right)$ and $c_{p}=\Phi\left(E_{\Gamma} ;-2 q\right)$, respectively. In the end, we have the 43 bosonic amplitudes listed in Table I.

\section{Single-particle basis}

The single-particle bases $\mathbf{h}_{s}$ and $\tilde{\mathbf{h}}_{s}$, parametrizing Eqs. (B11)-(B15), are block matrices,

$$
\mathbf{h}_{s}=\left(\begin{array}{cc}
h_{s} & h_{s}^{\prime} \\
h_{s}^{\prime \dagger} & -h_{s}^{*}
\end{array}\right) \quad \tilde{\mathbf{h}}_{s}=\left(\begin{array}{cc}
h_{s} & h_{s}^{\prime *} \\
h_{s}^{\prime} & h_{s}^{*}
\end{array}\right),
$$

where the component $h_{s}$ corresponds to the normal part and $h_{s}^{\prime}$ corresponds to the anomalous part of the matrix. The components for each fluctuation channel, in the degenerate three-orbital model, are as follows:

$$
\begin{aligned}
& h_{\mathrm{ch}}=\lambda_{0} \otimes \sigma_{0}, \\
& h_{\mathrm{sp}}=\lambda_{0} \otimes \sigma_{z}, \\
& h_{\mathrm{orb}}=\lambda_{4} \otimes \sigma_{0}, \\
& h_{\mathrm{so}}=\lambda_{4} \otimes \sigma_{z}, \\
& h_{\mathrm{orb}^{*}}=\lambda_{1} \otimes \sigma_{0}, \\
& h_{\mathrm{so}^{*}}=\lambda_{1} \otimes \sigma_{z}, \\
& h_{\mathrm{P}}=0
\end{aligned}
$$

\begin{tabular}{|c|c|c|c|}
\hline$(N, L, S)$ & Degeneracy & $E_{\Gamma}$ & $\Phi_{\Gamma n}$ \\
\hline$(0,0,0)$ & 1 & 0 & $\begin{array}{c}\Phi\left(E_{000}\right) \\
\Phi\left(E_{000} ; 2\right) \\
\Phi\left(E_{000} ; 4\right)\end{array}$ \\
\hline$\left(1,1, \frac{1}{2}\right)$ & 6 & 0 & $\begin{array}{c}\Phi\left(E_{11 \frac{1}{2}}\right) \\
\Phi\left(E_{11 \frac{1}{2}} ; 2\right) \\
\Phi\left(E_{11 \frac{1}{2}} ; 4\right)\end{array}$ \\
\hline$(2,2,0)$ & 5 & $U-J$ & $\begin{array}{c}\Phi\left(E_{220}\right) \\
\Phi\left(E_{220} ;-2\right) \\
\Phi\left(E_{220} ; 2\right) \\
\Phi\left(E_{220} ; 4\right)\end{array}$ \\
\hline$(2,1,1)$ & 9 & $U-3 J$ & $\begin{array}{c}\Phi\left(E_{211}\right) \\
\Phi\left(E_{211} ;-2\right) \\
\Phi\left(E_{211} ; 2\right)\end{array}$ \\
\hline$(2,0,0)$ & 1 & $U+2 J$ & $\begin{array}{c}\Phi\left(E_{200}\right) \\
\Phi\left(E_{200} ; 2\right) \\
\Phi\left(E_{200} ; 4\right)\end{array}$ \\
\hline$\left(3,2, \frac{1}{2}\right)$ & 10 & $3 U-6 J$ & $\begin{array}{c}\Phi\left(E_{32 \frac{1}{2}}\right) \\
\Phi\left(E_{32 \frac{1}{2}} ;-2\right) \\
\Phi\left(E_{32 \frac{1}{2}} ; 2\right)\end{array}$ \\
\hline$\left(3,1, \frac{1}{2}\right)$ & 6 & $3 U-4 J$ & $\begin{array}{c}\Phi\left(E_{31 \frac{1}{2}}\right) \\
\Phi\left(E_{31 \frac{1}{2}} ;-2\right) \\
\Phi\left(E_{31 \frac{1}{2}} ; 2\right)\end{array}$ \\
\hline$\left(3,0, \frac{3}{2}\right)$ & 4 & $3 U-9 J$ & $\begin{array}{c}\Phi\left(E_{30 \frac{3}{2}}\right) \\
\Phi\left(E_{30 \frac{3}{2}} ;-2\right) \\
\Phi\left(E_{30 \frac{3}{2}} ; 2\right)\end{array}$ \\
\hline$(4,2,0)$ & 5 & $6 U-11 J$ & $\begin{array}{c}\Phi\left(E_{420}\right) \\
\Phi\left(E_{420} ;-4\right) \\
\Phi\left(E_{420} ;-2\right) \\
\Phi\left(E_{420} ; 2\right)\end{array}$ \\
\hline$(4,1,1)$ & 9 & $6 U-13 J$ & $\begin{array}{c}\Phi\left(E_{411}\right) \\
\Phi\left(E_{411} ;-4\right) \\
\Phi\left(E_{411} ;-2\right) \\
\Phi\left(E_{411} ; 2\right)\end{array}$ \\
\hline$(4,0,0)$ & 1 & $U+2 J$ & $\begin{array}{c}\Phi\left(E_{400}\right) \\
\Phi\left(E_{400} ;-4\right) \\
\Phi\left(E_{400} ;-2\right) \\
\Phi\left(E_{400} ; 2\right)\end{array}$ \\
\hline$\left(5,1, \frac{1}{2}\right)$ & 6 & $10 U-20 J$ & $\begin{array}{c}\Phi\left(E_{51 \frac{1}{2}}\right) \\
\Phi\left(E_{51 \frac{1}{2}} ;-4\right) \\
\Phi\left(E_{51 \frac{1}{2}} ;-2\right)\end{array}$ \\
\hline$(6,0,0)$ & 1 & $15 U-30 J$ & $\begin{array}{c}\Phi\left(E_{600}\right) \\
\Phi\left(E_{600} ;-4\right) \\
\Phi\left(E_{600} ;-2\right)\end{array}$ \\
\hline
\end{tabular}

for the normal part, and

$$
\begin{gathered}
h_{\mathrm{ch}}^{\prime}=h_{\mathrm{sp}}^{\prime}=h_{\mathrm{orb}}^{\prime}=h_{\mathrm{so}}^{\prime}=h_{\mathrm{orb}^{*}}^{\prime}=h_{\mathrm{so}^{*}}^{\prime}=0, \\
h_{\mathrm{P}}^{\prime}=\lambda_{6} \otimes\left[-i \sigma_{y} \sigma_{z}\right]
\end{gathered}
$$

for the anomalous part, where the basis is chosen to be normalized, i.e., $\operatorname{Tr}\left[\mathbf{h}_{s} \mathbf{h}_{s}^{\dagger}\right]=1$. We see that $\mathbf{h}_{\mathrm{P}}$ describes the pairing fluctuation, while $\mathbf{h}_{\mathrm{ch}}, \mathbf{h}_{\mathrm{sp}}, \mathbf{h}_{\mathrm{orb}}, \mathbf{h}_{\mathrm{so}}, \mathbf{h}_{\mathrm{orb} *}$, and $\mathbf{h}_{\mathrm{so}^{*}}$
TABLE I. Quantum numbers $(N, L, S)$, degeneracy, eigenvalues, and the corresponding slave bosons $\Phi\left(E_{\Gamma} ; 2 q\right)$ for each local multiplet $|\Gamma\rangle$.

describe the charge, spin, orbital, and spin-orbital fluctuations, respectively.

\section{APPENDIX D: DERIVATION OF EQ. (37)}

The linear response for a generic operator is given by the following equation: 


$$
\begin{aligned}
\chi_{\mathcal{O O}} & =\left.\frac{d}{d \xi}\langle\Phi(\xi, \mathbf{x})|\hat{\mathcal{O}}| \Phi(\xi, \mathbf{x})\rangle\right|_{[\xi=0, \mathbf{x}(\xi=0)]} \\
& =\chi_{\mathcal{O O}}^{(0)}+\left.\sum_{\mu} \frac{d x_{\mu}}{d \xi}\right|_{\xi=0} \chi_{\mu \mathcal{O}} .
\end{aligned}
$$

Note again that $\mu$ runs through all the variational variables in $\mathbf{x}$ [Eq. (26)], and we use the variational parameters as the subscripts. To evaluate Eq. (D1), it is necessary to calculate $\left.\left(d x_{\mu} / d \xi\right)\right|_{\xi=0}$, which can be determined by taking the total derivative of Eq. (36) with respect to $\xi$, as follows:

$$
\left.\sum_{\nu} \mathcal{M}_{\mu \nu} \frac{d x_{\nu}}{d \xi}\right|_{\xi=0}-\chi_{\mu \mathcal{O}}=0
$$

where $\mathcal{M}$ is the fluctuation matrix defined in Eq. (40). Substituting Eq. (D2) into Eq. (D1), we obtain Eq. (37) in the main text. Since physical susceptibilities in Eq. (53) are gauge invariant, all solutions of Eq. (D2), connected by the gauge transformations [Eq. (H6)], are equivalent.

\section{APPENDIX E: FLUCTUATION MATRIX}

The fluctuation matrix can be separated into three parts:

$$
\mathcal{M}_{\mu \nu}(\mathbf{q})=\mathcal{M}_{\mu \nu}^{\mathrm{mix}}+\mathcal{M}_{\mu \nu}^{\mathrm{qp}}(\mathbf{q})+\mathcal{M}_{\mu \nu}^{\mathrm{emb}} .
$$

The first part, $\mathcal{M}^{\text {mix }}$, which involves the partial derivatives of the mixing term of the Lagrangian $\mathcal{L}_{\text {mix }}$ with respect to $r_{s}, l_{s}, d_{s}, D_{s}, l_{s}^{c}$, and $\zeta_{s}$, is computed from the following equations:

$$
\begin{aligned}
& \left.\mathcal{M}_{r_{s} d_{s^{\prime}}}^{\operatorname{mix}} \equiv \partial_{r_{s}} \partial_{d_{s^{\prime}}} \mathcal{L}_{\text {mix }}[\mathbf{x}]\right|_{[\xi=0, \mathbf{x}(\xi=0)]}=-\sum_{a \alpha c}\left(\mathbf{D}_{a \alpha} \tilde{\mathbf{h}}_{s, c \alpha} \partial_{d_{s^{\prime}}}[\mathbf{\Delta}(1-\mathbf{\Delta})]_{c a}^{\frac{1}{2}}+\text { c.c }\right), \\
& \left.\mathcal{M}_{r_{s} D_{s^{\prime}}}^{\text {mix }} \equiv \partial_{r_{s}} \partial_{D_{s^{\prime}}} \mathcal{L}_{\text {mix }}[\mathbf{x}]\right|_{[\xi=0, \mathbf{x}(\xi=0)]}=-\sum_{a \alpha c}\left(\tilde{\mathbf{h}}_{s, a \alpha} \tilde{\mathbf{h}}_{s^{\prime}, c \alpha}[\boldsymbol{\Delta}(1-\boldsymbol{\Delta})]_{c a}^{\frac{1}{2}}+\text { c.c }\right), \\
& \mathcal{M}_{l_{s} d_{s^{\prime}}}^{\operatorname{mix}}=\mathcal{M}_{d_{s} l_{s^{\prime}}^{c}}^{\text {mix }}=\mathcal{M}_{d_{s} \zeta_{s^{\prime}}}^{\operatorname{mix}}=-\frac{1}{2} \sum_{a b} \mathbf{h}_{a b}^{s}\left[\mathbf{h}^{s^{\prime}}\right]_{a b}^{t}, \\
& \left.\mathcal{M}_{d_{s} d_{s^{\prime}}}^{\text {mix }} \equiv \partial_{d_{s}} \partial_{d_{s^{\prime}}} \mathcal{L}_{\text {mix }}[\mathbf{x}]\right|_{[\xi=0, \mathbf{x}(\xi=0)]}=-\sum_{a \alpha c}\left(\mathbf{D}_{a \alpha} \mathbf{R}_{c \alpha} \partial_{d_{s}} \partial_{d_{s^{\prime}}}[\boldsymbol{\Delta}(1-\mathbf{\Delta})]_{c a}^{\frac{1}{2}}+\text { c.c. }\right), \\
& \left.\mathcal{M}_{d_{s} D_{s^{\prime}}}^{\text {mix }} \equiv \partial_{d_{s}} \partial_{D_{s^{\prime}}} \mathcal{L}_{\text {mix }}[\mathbf{x}]\right|_{[\xi=0, \mathbf{x}(\xi=0)]}=-\sum_{a \alpha c}\left(\tilde{\mathbf{h}}_{s^{\prime}, a \alpha} \mathbf{R}_{c \alpha} \partial_{d_{s}}[\boldsymbol{\Delta}(1-\boldsymbol{\Delta})]_{c a}^{\frac{1}{2}}+\text { c.c }\right),
\end{aligned}
$$

and the other unlisted components of $\mathcal{M}^{\text {mix }}$ are zero.

The second part $\mathcal{M}^{\mathrm{qp}}$, which involves the partial derivatives of the quasiparticle term of the Lagrangian $\mathcal{L}_{\mathrm{qp}}$ with respect to $r_{s}$ and $l_{s}$, is computed from the following equations:

$$
\begin{gathered}
\mathcal{M}_{r_{s} r_{s^{\prime}}}^{\mathrm{qp}}(q)=\left.\partial_{r_{s, q}} \partial_{r_{s^{\prime}, q}} \mathcal{L}_{\mathrm{qp}}[\mathbf{x}]\right|_{[\xi=0, \mathbf{x}(\xi=0)]}=\frac{1}{2 N} \sum_{\mathbf{k}} \operatorname{Tr}\left\{n_{F}\left(H_{\mathbf{k}}^{\mathrm{qp}}\right)\left[\tilde{\mathbf{h}}_{s} \tilde{\mathbf{\epsilon}}_{\mathbf{k}+\mathbf{q}} \tilde{\mathbf{h}}_{s^{\prime}}^{\dagger}+\tilde{\mathbf{h}}_{s^{\prime}} \tilde{\mathbf{\epsilon}}_{\mathbf{k}-\mathbf{q}} \tilde{\mathbf{h}}_{s}^{\dagger}\right]+T \sum_{\omega_{n}} \mathbf{G}_{k}[\mathbf{R}]^{-1}\left[\mathbf{R} \tilde{\mathbf{\epsilon}}_{\mathbf{k}} \tilde{\mathbf{h}}_{s}^{\dagger}+\tilde{\mathbf{h}}_{s} \tilde{\mathbf{\epsilon}}_{\mathbf{k}+\mathbf{q}} \mathbf{R}^{\dagger}\right]\left[\mathbf{R}^{\dagger}\right]^{-1} \mathbf{G}_{k+q}\right. \\
\left.\cdot[\mathbf{R}]^{-1}\left[\mathbf{R} \tilde{\mathbf{\epsilon}}_{\mathbf{k}+\mathbf{q}} \tilde{\mathbf{h}}_{s^{\prime}}^{\dagger}+\tilde{\mathbf{h}}_{s^{\prime}} \tilde{\mathbf{\epsilon}}_{\mathbf{k}} \mathbf{R}^{\dagger}\right]\left[\mathbf{R}^{\dagger}\right]^{-1}\right\}, \\
\left.\mathcal{M}_{r_{s} l_{s^{\prime}}}^{\mathrm{qp}}(q) \equiv \partial_{r_{s, q}} \partial_{l_{s^{\prime}, q}} \mathcal{L}_{\mathrm{qp}}[\mathbf{x}]\right|_{[\xi=0, \mathbf{x}(\xi=0)]} \\
=\frac{T}{2 N} \sum_{k} \operatorname{Tr}\left\{\mathbf{G}_{k}[\mathbf{R}]^{-1}\left[\mathbf{R} \tilde{\mathbf{\epsilon}}_{\mathbf{k}} \tilde{\mathbf{h}}_{s}^{\dagger}+\tilde{\mathbf{h}}_{s} \tilde{\mathbf{\epsilon}}_{\mathbf{k}+\mathbf{q}} \mathbf{R}^{\dagger}\right] \cdot\left[\mathbf{R}^{\dagger}\right]^{-1} \mathbf{G}_{k+q}[\mathbf{R}]^{-1} \mathbf{h}_{s^{\prime}}\left[\mathbf{R}^{\dagger}\right]^{-1}\right\}, \\
\left.\mathcal{M}_{l_{s} l_{s^{\prime}}}^{\mathrm{qp}}(q) \equiv \partial l_{s,-q} \partial l_{s^{\prime}, q} \mathcal{L}_{\mathrm{qp}}[\mathbf{x}]\right|_{[\xi=0, \mathbf{x}(\xi=0)]} \\
=\frac{T}{2 N} \sum_{k} \operatorname{Tr}\left\{\mathbf{G}_{k}[\mathbf{R}]^{-1} \mathbf{h}_{s}\left[\mathbf{R}^{\dagger}\right]^{-1} \cdot \mathbf{G}_{k+q}[\mathbf{R}]^{-1} \mathbf{h}_{s^{\prime}}\left[\mathbf{R}^{\dagger}\right]^{-1}\right\},
\end{gathered}
$$

and the other unlisted components of $\mathcal{M}^{\mathrm{qp}}$ are zero. We also define $k=\left(\omega_{n}, \mathbf{k}\right)$ and $\sum_{k} \equiv \sum_{\mathbf{k}} \sum_{\omega_{n}}$. Note that since we consider the degenerate three-orbital model, at the normal-state saddle point, the renormalization matrix, the local potential, the quasiparticle energy dispersion, and the Green's functions are all degenerate and diagonal matrices, i.e., 


$$
\begin{gathered}
\mathbf{R}=R_{0}\left(\begin{array}{cc}
I & 0 \\
0 & I
\end{array}\right), \\
\boldsymbol{\Lambda}=l_{0}\left(\begin{array}{cc}
I & 0 \\
0 & -I
\end{array}\right), \\
H_{\mathbf{k}}^{\mathrm{qp}}=E_{\mathbf{k}}^{\mathrm{qp}}\left(\begin{array}{cc}
I & 0 \\
0 & -I
\end{array}\right), \\
\mathbf{G}^{\mathrm{qp}}(k)=\left(\begin{array}{cc}
\frac{1}{i \omega_{n}-E_{\mathbf{k}}^{\mathrm{qp}}} I & 0 \\
0 & \frac{1}{-i \omega_{n}-E_{\mathbf{k}}^{\mathrm{qp}}} I
\end{array}\right),
\end{gathered}
$$

where $E_{\mathbf{k}}^{\mathrm{qp}}=R_{0}^{2} \epsilon_{\mathbf{k}}+l_{0}$ and $I$ is the $6 \times 6$ identity matrix. The Matsubara summation for the fermionic Green's function convolutions in $\mathcal{M}_{r r}^{\mathrm{qp}}, \mathcal{M}_{r l}^{\mathrm{qp}}$, and $\mathcal{M}_{l l}^{\mathrm{qp}}$ can be evaluated analytically from the Lindhard function. For example, the particlehole convolution is

$$
T \sum_{\omega_{m}} \frac{1}{i \omega_{m}-E_{\mathbf{k}}^{\mathrm{qp}}} \frac{1}{i \omega_{m}+i \Omega_{n}-E_{\mathbf{k}+\mathbf{q}}^{\mathrm{qp}}}=\frac{n_{F}\left(E_{\mathbf{k}}^{\mathrm{qp}}\right)-n_{F}\left(E_{\mathbf{k}+\mathbf{q}}^{\mathrm{qp}}\right)}{i \Omega_{n}-E_{\mathbf{k}+\mathbf{q}}^{\mathrm{qp}}+E_{\mathbf{k}}^{\mathrm{qp}}},
$$

and the particle-particle convolution is

$$
T \sum_{\omega_{m}} \frac{1}{i \omega_{m}+i \Omega_{n}-E_{\mathbf{k}+\mathbf{q}}^{\mathrm{qp}}} \frac{1}{-i \omega_{m}-E_{-\mathbf{k}}^{\mathrm{qp}}}=\frac{n_{F}\left(E_{\mathbf{k}+\mathbf{q}}^{\mathrm{qp}}\right)-n_{F}\left(-E_{-\mathbf{k}}^{\mathrm{qp}}\right)}{i \Omega_{n}-E_{\mathbf{k}+\mathbf{q}}^{\mathrm{qp}}-E_{-\mathbf{k}}^{\mathrm{qp}}} .
$$

The analytical continuation to real frequency can be achieved by the replacement $i \Omega_{n} \rightarrow \omega+i 0^{+}$.

The third part, $\mathcal{M}^{\mathrm{emb}}$, involves the partial derivatives of the embedding term of the Lagrangian $\mathcal{L}_{\text {emb }}$ with respect to $D_{s}$, $l_{s}^{c}$, and $\zeta_{s}$, which can be evaluated as follows. First, we evaluate the first-order derivatives using the Hellmann-Feynman theorem:

$$
\begin{gathered}
\partial_{l_{s}^{c}} \mathcal{L}_{\mathrm{emb}}[\xi, \mathbf{x}]=\sum_{a b c d} \frac{1}{2} \mathbf{h}_{a b}^{s}\left\langle\Phi(\xi, \mathbf{x})\left|\bar{I}_{b c} \hat{\Psi}_{c} \hat{\Psi}_{d}^{\dagger} \bar{I}_{d a}\right| \Phi(\xi, \mathbf{x})\right\rangle, \\
\partial_{D_{s}} \mathcal{L}_{\mathrm{emb}}[\xi, \mathbf{x}]=2 \sum_{a \alpha b} \tilde{\mathbf{h}}_{a \alpha}^{s}\left\langle\Phi(\xi, \mathbf{x})\left|\hat{\Xi}_{\alpha}^{\dagger} \hat{\Psi}_{b} \bar{I}_{b a}\right| \Phi(\xi, \mathbf{x})\right\rangle, \\
\partial_{\zeta_{s}} \mathcal{L}_{\mathrm{emb}}[\xi, \mathbf{x}]=\frac{1}{2} \sum_{\alpha \beta} \mathbf{h}_{\alpha \beta}^{s}\left\langle\Phi(\xi, \mathbf{x})\left|\hat{\Xi}_{\alpha}^{\dagger} \hat{\Xi}_{\beta}\right| \Phi(\xi, \mathbf{x})\right\rangle .
\end{gathered}
$$

Then, we compute the second-order derivatives from the following equations:

$$
\begin{gathered}
\mathcal{M}_{l_{s}^{c} c_{s^{\prime}}}^{\mathrm{emb}}=\left.\partial_{l_{s}^{c}} \partial_{l_{s^{\prime}}} \mathcal{L}_{\mathrm{emb}}[\xi, \mathbf{x}]\right|_{[\xi=0, \mathbf{x}(\xi=0)]}=\left.\partial_{l_{s}^{c}} \sum_{a b c d} \frac{1}{2} \mathbf{h}_{a b}^{s^{\prime}}\left\langle\Phi(\xi, \mathbf{x})\left|\bar{I}_{b c} \hat{\Psi}_{c} \hat{\Psi}_{d}^{\dagger} \bar{I}_{d a}\right| \Phi(\xi, \mathbf{x})\right\rangle\right|_{[\xi=0, \mathbf{x}(\xi=0)]}, \\
\mathcal{M}_{l_{s}^{c} D_{s^{\prime}}}^{\mathrm{emb}}=\left.\partial_{l_{s}^{c}} \partial_{D_{s^{\prime}}} \mathcal{L}_{\mathrm{emb}}[\xi, \mathbf{x}]\right|_{[\xi=0, \mathbf{x}(\xi=0)]}=\left.\partial_{l_{s}^{c}} 2 \sum_{a \alpha b} \tilde{\mathbf{h}}_{a \alpha}^{s^{\prime}}\left\langle\Phi(\xi, \mathbf{x})\left|\hat{\Xi}_{\alpha}^{\dagger} \hat{\Psi}_{b} \bar{I}_{b a}\right| \Phi(\xi, \mathbf{x})\right\rangle\right|_{[\xi=0, \mathbf{x}(\xi=0)]}, \\
\mathcal{M}_{D_{s}, l_{s^{\prime}}^{c}}^{\mathrm{emb}}=\left.\partial_{D_{s}} \partial_{l_{s^{\prime}}^{c}} \mathcal{L}_{\mathrm{emb}}[\xi, \mathbf{x}]\right|_{[\xi=0, \mathbf{x}(\xi=0)]}=\left.\partial_{D_{s}} \frac{1}{2} \sum_{a b c d} \mathbf{h}_{a b}^{s^{\prime}}\left\langle\Phi(\xi, \mathbf{x})\left|\bar{I}_{b c} \hat{\Psi}_{c} \hat{\Psi}_{d}^{\dagger} \bar{I}_{d a}\right| \Phi(\xi, \mathbf{x})\right\rangle\right|_{[\xi=0, \mathbf{x}(\xi=0)]}, \\
\mathcal{M}_{D_{s} D_{s^{\prime}}}^{\mathrm{emb}}=\left.\partial_{D_{s}} \partial_{D_{s^{\prime}}} \mathcal{L}_{\mathrm{emb}}[\xi, \mathbf{x}]\right|_{[\xi=0, \mathbf{x}(\xi=0)]}=\left.\partial_{D_{s}} 2 \sum_{a \alpha b} \tilde{\mathbf{h}}_{a \alpha}^{s^{\prime}}\left\langle\Phi(\xi, \mathbf{x})\left|\hat{\Xi}_{\alpha}^{\dagger} \hat{\Psi}_{b} \bar{I}_{b a}\right| \Phi(\xi, \mathbf{x})\right\rangle\right|_{[\xi=0, \mathbf{x}(\xi=0)]},
\end{gathered}
$$




$$
\begin{gathered}
\mathcal{M}_{D_{s} \zeta_{s^{\prime}}}^{\mathrm{emb}}=\left.\partial_{l_{s}^{c}} \partial_{\zeta_{s^{\prime}}} \mathcal{L}_{\mathrm{emb}}[\xi, \mathbf{x}]\right|_{[\xi=0, \mathbf{x}(\xi=0)]}=\partial_{D_{s}} \frac{1}{2} \sum_{\alpha \beta} \mathbf{h}_{\alpha \beta}^{s^{\prime}}\left\langle\left.\Phi(\xi, \mathbf{x})\left|\hat{\Xi}_{\alpha}^{\dagger} \hat{\Xi}_{\beta}\right| \Phi(\xi, \mathbf{x})\right|_{[\xi=0, \mathbf{x}(\xi=0)]},\right. \\
\mathcal{M}_{\zeta_{s}, l_{s^{\prime}}}^{\mathrm{emb}}=\left.\partial_{\zeta_{s}} \partial_{l_{s^{\prime}}} \mathcal{L}_{\mathrm{emb}}[\xi, \mathbf{x}]\right|_{[\xi=0, \mathbf{x}(\xi=0)]}=\left.\partial_{\zeta_{s}} \frac{1}{2} \sum_{a b c d} \mathbf{h}_{a b}^{s^{\prime}}\left\langle\Phi(\xi, \mathbf{x})\left|\bar{I}_{b c} \hat{\Psi}_{c} \hat{\Psi}_{d}^{\dagger} \bar{I}_{d a}\right| \Phi(\xi, \mathbf{x})\right\rangle\right|_{[\xi=0, \mathbf{x}(\xi=0)]}, \\
\mathcal{M}_{\zeta_{s} D_{s^{\prime}}}^{\mathrm{emb}}=\left.\partial_{\zeta_{s}} \partial_{D_{s^{\prime}}} \mathcal{L}_{\mathrm{emb}}[\xi, \mathbf{x}]\right|_{[\xi=0, \mathbf{x}(\xi=0)]}=\left.\partial_{\zeta_{s}} 2 \sum_{a \alpha b} \tilde{\mathbf{h}}_{a \alpha}^{s^{\prime}}\left\langle\Phi(\xi, \mathbf{x})\left|\hat{\Xi}_{\alpha}^{\dagger} \hat{\Psi}_{b} \bar{I}_{b a}\right| \Phi(\xi, \mathbf{x})\right\rangle\right|_{[\xi=0, \mathbf{x}(\xi=0)]}, \\
\mathcal{M}_{\zeta_{\zeta_{s}} \zeta_{s^{\prime}}}^{\mathrm{emb}}=\left.\partial_{\zeta_{s}} \partial_{\zeta_{s^{\prime}}} \mathcal{L}_{\mathrm{emb}}[\xi, \mathbf{x}]\right|_{[\xi=0, \mathbf{x}(\xi=0)]}=\left.\partial_{\zeta_{s}} \frac{1}{2} \sum_{\alpha \beta} \mathbf{h}_{\alpha \beta}^{s^{\prime}}\left\langle\Phi(\xi, \mathbf{x})\left|\hat{\Xi}_{\alpha}^{\dagger} \hat{\Xi}_{\beta}\right| \Phi(\xi, \mathbf{x})\right\rangle\right|_{[\xi=0, \mathbf{x}(\xi=0)]},
\end{gathered}
$$

where the other unlisted components of $\mathcal{M}^{\mathrm{emb}}$ are zero.

The above second-order derivatives and Eqs. (38) and (39) can be evaluated using the linear response theory. We apply a perturbation to the embedding Hamiltonian

$$
\hat{H}_{\mathrm{emb}}(\eta)=\hat{H}_{\mathrm{emb}}+\eta \hat{A},
$$

where $\hat{A}=\sum_{a b c d} \mathbf{h}_{a b}^{s} \bar{I}_{b c} \hat{\Psi}_{c} \hat{\Psi}_{d}^{\dagger} \bar{I}_{d a}, \sum_{a \alpha b} \tilde{\mathbf{h}}_{a \alpha}^{s} \hat{\Xi}_{\alpha}^{\dagger} \hat{\Psi}_{b} \bar{I}_{b a}$, or $\hat{\mathcal{O}}$ corresponding to the perturbation in $\eta=l_{s}^{c}, D_{s}$, or $\xi$, respectively. We want to compute the change in the average of $\langle\hat{B}\rangle_{\eta}$ in the limit $\eta \rightarrow 0$, where $\hat{B}=$ $\sum_{a b c d} \mathbf{h}_{a b}^{s} \bar{I}_{b c} \hat{\Psi}_{c} \hat{\Psi}_{d}^{\dagger} \bar{I}_{d a}, \quad \sum_{a \alpha b} \tilde{\mathbf{h}}_{a \alpha}^{s} \hat{\Xi}_{\alpha}^{\dagger} \hat{\Psi}_{b} \bar{I}_{b a}$, or $\hat{\mathcal{O}}$. This response function can be computed from the spectral representation of the static susceptibility at zero temperature:

$$
\begin{aligned}
\left.\frac{\partial\langle\hat{B}\rangle_{\eta}}{\partial \eta}\right|_{\eta=0} & =\chi_{\hat{A} \hat{B}} \\
& =\lim _{\epsilon \rightarrow 0^{+}} \sum_{n}\left[\frac{\langle 0|\hat{A}| n\rangle\langle n|\hat{B}| 0\rangle}{E_{n}-E_{0}+i \epsilon}-\frac{\langle 0|\hat{B}| n\rangle\langle n|\hat{A}| 0\rangle}{E_{0}-E_{n}+i \epsilon}\right],
\end{aligned}
$$

where $E_{n}$ is the $n$th excited-state energy of $\hat{H}_{\text {emb }}$ and $|n\rangle$ is the $n$th excited-state wave function of $\hat{H}_{\text {emb }}$.

Besides the method proposed in Eqs. (E28), one can also use the finite difference method to evaluate the partial derivatives in Eqs. (E19)-(E26). Note that both methods require the diagonalization of the embedding Hamiltonian $\hat{H}_{\text {emb }}$, which is the most time-consuming part of the linearresponse calculations. With the current state of the art, we can easily study the $f$-electron materials, which contain seven correlated orbitals, using exact-diagonalization and machine-learning techniques [90]. For the systems with more correlated orbitals, one may also utilize the density matrix renormalization group or auxiliary-field quantum Monte Carlo methods [91].

\section{APPENDIX F: FLUCTUATION MATRIX AS A BOSONIC PROPAGATOR}

Here, we discuss how the fluctuation matrix can be interpreted as the propagator for the fluctuations of the bosonic variables $\mathbf{x}_{i}$. Let us expand the Lagrangian, Eq. (46), to the second order in

$$
\begin{gathered}
\delta \mathbf{x}_{i}^{t}=\left(\delta r_{\mathrm{ch}}, \delta l_{\mathrm{ch}}, \delta d_{\mathrm{ch}}, \delta D_{\mathrm{ch}}, \delta l_{s}^{c}, \delta \zeta_{\mathrm{ch}}, \ldots, \delta r_{s}, \delta l_{s}, \delta d_{s},\right. \\
\left.\delta D_{s}, \delta l_{s}^{c}, \delta \zeta_{s}, \ldots, \delta r_{\mathrm{P}}, \delta l_{\mathrm{P}}, \delta d_{\mathrm{P}}, \delta D_{\mathrm{P}}, \delta l_{\mathrm{P}}^{c}, \delta \zeta_{\mathrm{P}}\right)
\end{gathered}
$$

around the normal-state saddle point:

$$
\begin{aligned}
\mathcal{L}^{s}\left[\delta \mathbf{x}, \Xi, \Xi^{\dagger}\right]= & \frac{T}{2 N} \sum_{k} \sum_{\alpha \beta} \Xi_{\mathbf{k} \alpha}^{\dagger}[\mathbf{G}(k)]_{\alpha \beta}^{-1} \Xi_{\mathbf{k} \beta} \\
& +\frac{1}{2} \sum_{i} \delta \mathbf{x}_{i}^{t}\left[\mathcal{M}^{\text {mix }}+\mathcal{M}^{\mathrm{emb}}\right] \delta \mathbf{x}_{i,} \\
& +\sum_{\mathbf{k}, \mathbf{q}} \sum_{\alpha \beta}\left[\left(\tilde{\Lambda}_{\alpha \beta, r_{s}}^{\mathbf{k}, \mathbf{q}} \delta r_{s, \mathbf{q}} \Xi_{\mathbf{k}+\mathbf{q} \alpha}^{\dagger} \Xi_{\mathbf{k} \beta}\right.\right. \\
& \left.+ \text { H.c. })+\tilde{\Lambda}_{\alpha \beta, l_{s}} \delta l_{s, \mathbf{q}} \Xi_{\mathbf{k}+\mathbf{q} \alpha}^{\dagger} \Xi_{\mathbf{k} \beta}\right] \\
& +\sum_{\mathbf{k}, \mathbf{k}^{\prime}, \mathbf{q}} \sum_{\alpha \beta} \tilde{\gamma}_{\alpha \beta, r_{s}, \mathbf{k}_{s^{\prime}}}^{\mathbf{k}} \delta r_{s, \mathbf{q}} \delta r_{s^{\prime},-\mathbf{q}} \Xi_{\mathbf{k} \alpha}^{\dagger} \Xi_{\mathbf{k}^{\prime} \beta},
\end{aligned}
$$

where $\tilde{\Lambda}_{\alpha \beta \mu}$ are the three-leg vertices defined in Eqs. (60) and (61) and $\mathbf{G}(k)$ is the Nambu propagator. We also introduce the four-leg vertex:

$\tilde{\gamma}_{\alpha \beta r_{s} r_{s^{\prime}}}^{\mathbf{k}, \mathbf{k}^{\prime}, \mathbf{q}}=\frac{1}{2}\left[\mathbf{R}^{-1}\left(\tilde{\mathbf{h}}_{s} \tilde{\mathbf{\epsilon}}_{\mathbf{k}+\mathbf{q}} \tilde{\mathbf{h}}_{s^{\prime}}^{\dagger}+\tilde{\mathbf{h}}_{s^{\prime}} \tilde{\mathbf{\epsilon}}_{\mathbf{k}^{\prime}-\mathbf{q}} \tilde{\mathbf{h}}_{s}^{\dagger}\right)\left(\mathbf{R}^{\dagger}\right)^{-1}\right]_{\alpha \beta}$.

We immediately see that the $\mathbf{q}$ independent part of the fluctuation matrix,

$$
\mathcal{M}^{\text {mix }}+\mathcal{M}^{\mathrm{emb}} \equiv \mathcal{D}_{0}^{-1},
$$


Particle-hole channel: $s \in\{\mathrm{ch}, \mathrm{sp}$, orb, so, orb*, so* $\}$

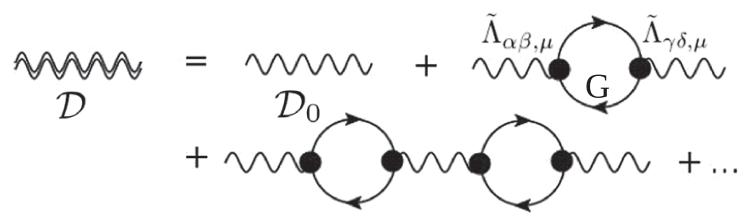

Particle-particle channel: $s=P$

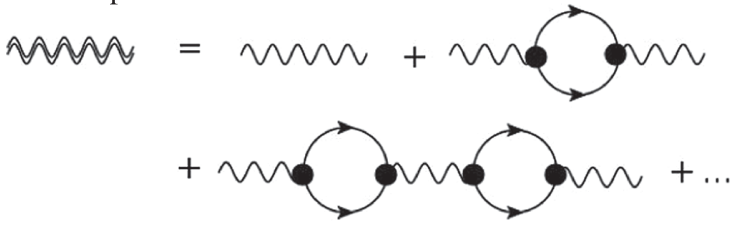

FIG. 8. Diagrammatic representation of the Dyson equation in Eq. (F5). The double wavy line and the wavy line denote the dressed bosonic propagator $\mathcal{D}$ and the bare bosonic propagator $\mathcal{D}_{0}$. The solid line denotes the Nambu propagator $\mathbf{G}$. The circle denotes the three-leg vertices $\tilde{\Lambda}$.

can be viewed, in the Gaussian fluctuation sense [38], as the inverse of the bare bosonic propagator $\mathcal{D}_{0}^{-1}$. It is important to note that $\mathcal{D}_{0}$ describes the local multiplet fluctuations because it contains the embedding susceptibilities shown in Eqs. (E19)-(E26). We see that, for the pairing channel $s=$ $\mathrm{P}$ in Eqs. (E19)-(E26), the multiplet fluctuation selects the basis $\mathbf{h}_{\mathrm{P}}$ that increases and removes electron pairs from the saddle-point wave function. Therefore, it describes the local fluctuation with pair excitations. On the other hand, for channel $s \in\{\mathrm{ch}, \mathrm{sp}$, orb, so, orb*, so*\}, the particle number is conserved. Consequently, they describe the corresponding local charge, orbital, and spin fluctuations.

We now discuss the role of $\mathcal{M}^{\mathrm{qp}}(\mathbf{q})$. By integrating out the fermionic field $\Xi_{\mathbf{k} \alpha}$ in Eq. (F2) to the one-loop order, we find that the self-energy correction is related to the fluctuation matrix through $\pi(\mathbf{q}) \equiv-\mathcal{M}^{\mathrm{qp}}(\mathbf{q})$. Therefore, we can write the total fluctuation matrix in terms of the Dyson equation:

$$
\mathcal{M}(\mathbf{q}) \equiv \mathcal{D}^{-1}(\mathbf{q})=\mathcal{D}_{0}^{-1}-\pi(\mathbf{q}) .
$$

The total fluctuation matrix corresponds to the dressed bosonic propagator with the self-energy correction summing the fermionic bubbles to the infinite order. From Eq. (E7)(E9), we see that $\mathcal{M}^{\text {qp }}$ contains only the particle-particle bubbles for the pairing channel $s=P$ and the particle-hole bubbles for the other channels $s \in\{\mathrm{ch}, \mathrm{sp}$, orb, so, orb*, so* $^{*}$. Figure 8 shows the diagrammatic representation of the Dyson equation for the particle-hole and the particleparticle channels.

\section{APPENDIX G: RANDOM PHASE APPROXIMATION FOR THE INTERACTION VERTEX}

In this section, we derive the random phase approximation for the interaction vertex at $\mathbf{q}=0$. Therefore, we suppress the $\mathbf{q}$ dependent of $\tilde{\Gamma}, \tilde{\lambda}, \mathcal{D}$, and $\pi$ in the following derivation. The interaction vertex has the following form [see Eqs. (64) and (F5)]:

$$
\begin{aligned}
\tilde{\Gamma}_{\alpha \beta \gamma \delta}^{s}\left(\mathbf{k}, \mathbf{k}^{\prime}\right)= & -4\left[\tilde{\Lambda}_{\alpha \beta r_{s}}(\mathbf{k}) \mathcal{D}_{r_{s} r_{s}} \tilde{\Lambda}_{\gamma \delta r_{s}}\left(\mathbf{k}^{\prime}\right)\right. \\
& +2 \tilde{\Lambda}_{\alpha \beta r_{s}}(\mathbf{k}) \tilde{\Lambda}_{\gamma \delta l_{s}}\left(\mathbf{k}^{\prime}\right) \mathcal{D}_{r_{s} l_{s}} \\
& \left.+\tilde{\Lambda}_{\alpha \beta l_{s}}(\mathbf{k}) \tilde{\Lambda}_{\gamma \delta l_{s}}\left(\mathbf{k}^{\prime}\right) \mathcal{D}_{l_{s} l_{s}}\right]
\end{aligned}
$$

where $\tilde{\Lambda}_{\mu}$ is the three-leg vertex and $\mathcal{D} \equiv \mathcal{M}^{-1}$ is the bosonic Green's function defined in Eq. (F5). We want to obtain a RPA-like form for the vertex:

$$
\tilde{\Gamma}_{\alpha \beta \gamma \delta}^{s} \equiv\left\langle\left\langle\tilde{\Gamma}_{\alpha \beta \gamma \delta}^{s}\left(\mathbf{k}, \mathbf{k}^{\prime}\right)\right\rangle_{\mathbf{k}_{F}}\right\rangle_{\mathbf{k}_{F}^{\prime}}=\frac{F_{s}}{1+F_{s} \chi_{\mathcal{O}_{s} \mathcal{O}_{s}}^{(0)}}\left[\mathbf{h}_{s}\right]_{\alpha \beta}\left[\mathbf{h}_{s}\right]_{\gamma \delta}
$$

after averaging $\mathbf{k}$ and $\mathbf{k}^{\prime}$ over the Fermi surface, where $F_{s}$ is the Landau parameter.

We know that the bosonic Green's function has the following Dyson form for each sector $s$ [see Eq. (F5)]:

$$
\begin{aligned}
\mathcal{D}_{s} & =\left[\left[\mathcal{D}_{0, s}\right]^{-1}-\pi_{s}\right]^{-1} \\
& =\left[1-\mathcal{D}_{0, s} \pi\right]^{-1} \mathcal{D}_{0, s},
\end{aligned}
$$

where $\mathcal{D}_{0}$ is the bare bosonic propagator, and the selfenergy in each sector $s$ has the form

$$
\pi_{s}=\left(\begin{array}{cccccc}
\pi_{r_{s} r_{s}} & \pi_{r_{s} l_{s}} & 0 & 0 & 0 & 0 \\
\pi_{r_{s} l_{s}} & \pi_{l_{s} l_{s}} & 0 & 0 & 0 & 0 \\
0 & 0 & 0 & 0 & 0 & 0 \\
0 & 0 & 0 & 0 & 0 & 0 \\
0 & 0 & 0 & 0 & 0 & 0 \\
0 & 0 & 0 & 0 & 0 & 0
\end{array}\right) .
$$

The divergence of $\mathcal{D}$ can be determined from

$$
\begin{aligned}
\operatorname{Det}\left[1-\mathcal{D}_{0, s} \pi\right]= & 1-\mathcal{D}_{0, l_{s} l_{s}} \pi_{l_{s} l_{s}}-2 \mathcal{D}_{0, r_{s} l_{s}} \pi_{r_{s} l_{s}} \\
& -\mathcal{D}_{0, r_{s} r_{s}} \pi_{r_{s} r_{s}}+\left(\mathcal{D}_{0, r_{s} l_{s}} \pi_{r_{s} l_{s}}\right)^{2} \\
& -\mathcal{D}_{0, l_{s} l_{s}} \mathcal{D}_{0, r_{s} r_{s}} \pi_{r_{s} l_{s}}^{2}-\left(\mathcal{D}_{0, r_{s} l_{s}}\right)^{2} \pi_{l_{s} l_{s}} \pi_{r_{s} r_{s}} \\
& +\mathcal{D}_{0, l_{s} l_{s}} \mathcal{D}_{0, r_{s} r_{s}} \pi_{l_{s} l_{s}} \pi_{r_{s} r_{s}}=0 .
\end{aligned}
$$

The interaction vertex can be expressed in terms of $\mathcal{D}_{0}, \tilde{\Lambda}$, and $\pi$ as 


$$
\begin{aligned}
\tilde{\Gamma}_{\alpha \beta \gamma \delta}^{s}\left(\mathbf{k}, \mathbf{k}^{\prime}\right)= & -\frac{4}{\operatorname{Det}\left[1-\mathcal{D}_{0, s} \pi\right]}\left[\tilde{\Lambda}_{\alpha \beta r_{s}}(\mathbf{k}) \tilde{\Lambda}_{\gamma \delta r_{s}}\left(\mathbf{k}^{\prime}\right)\left(\mathcal{D}_{0, r_{s} r_{s}}+\left(\mathcal{D}_{0, r_{s} l_{s}}\right)^{2} \pi_{l_{s} l_{s}}-\mathcal{D}_{0, l_{s} l_{s}} \mathcal{D}_{0, r_{s} r_{s}} \pi_{l_{s} l_{s}}\right)\right. \\
& +2 \tilde{\Lambda}_{\alpha \beta r_{s}}(\mathbf{k}) \tilde{\Lambda}_{\gamma \delta l_{s}}\left(\mathbf{k}^{\prime}\right)\left(\mathcal{D}_{0, r_{s} l_{s}}-\left(\mathcal{D}_{0, r_{s} l_{s}}\right)^{2} \pi_{r_{s} l_{s}}+\mathcal{D}_{0, l_{s} l_{s}} \mathcal{D}_{0, r_{s} r_{s}} \pi_{r_{s} l_{s}}\right)+\tilde{\Lambda}_{\alpha \beta l_{s}}(\mathbf{k}) \tilde{\Lambda}_{\gamma \delta l_{s}}\left(\mathbf{k}^{\prime}\right) \\
& \left.\times\left(\mathcal{D}_{0, l_{s} l_{s}}+\left(\mathcal{D}_{0, r_{s} l_{s}}\right)^{2} \pi_{r_{s} r_{s}}-\mathcal{D}_{0, l_{s} l_{s}} \mathcal{D}_{0, r_{s} r_{s}} \pi_{r_{s} r_{s}}\right)\right]
\end{aligned}
$$

We can make a further approximation that

$$
\begin{gathered}
\pi_{r_{s} r_{s^{\prime}}}=\left.\frac{-T}{2 N} \sum_{k} \operatorname{tr}\left\{\mathbf{G}_{k}[\mathbf{R}]^{-1}\left[\mathbf{R} \boldsymbol{\epsilon}_{\mathbf{k}} \tilde{\mathbf{h}}_{s}^{\dagger}+\tilde{\mathbf{h}}_{s} \mathbf{\epsilon}_{\mathbf{k}} \mathbf{R}^{\dagger}\right]\left[\mathbf{R}^{\dagger}\right]^{-1} \mathbf{G}_{k}[\mathbf{R}]^{-1}\left[\mathbf{R} \mathbf{\epsilon}_{\mathbf{k}} \tilde{\mathbf{h}}_{s^{\prime}}^{\dagger}+\tilde{\mathbf{h}}_{s^{\prime}} \mathbf{\epsilon}_{\mathbf{k}} \mathbf{R}^{\dagger}\right]\left[\mathbf{R}^{\dagger}\right]^{-1}\right\}\right|_{\xi=0} \\
\approx 4\left\langle\tilde{\Lambda}_{r}\right\rangle^{2} \chi_{\mathcal{O}_{s} \mathcal{O}_{s}}^{(0)}, \\
\left.\pi_{r_{s} l_{s^{\prime}}}=\frac{-T}{2 N} \sum_{k} \operatorname{tr}\left\{\mathbf{G}_{k}[\mathbf{R}]^{-1}\left[\mathbf{R} \mathbf{\epsilon}_{\mathbf{k}} \tilde{\mathbf{h}}_{s}^{\dagger}+\tilde{\mathbf{h}}_{s} \mathbf{\epsilon}_{\mathbf{k}} \mathbf{R}^{\dagger}\right]\left[\mathbf{R}^{\dagger}\right]^{-1} \mathbf{G}_{k}[\mathbf{R}]^{-1} \mathbf{h}_{s^{\prime}}\left[\mathbf{R}^{\dagger}\right]\right]^{-1}\right\}\left.\right|_{\xi=0} \\
\approx 4\left\langle\tilde{\Lambda}_{r}\right\rangle\left\langle\tilde{\Lambda}_{l}\right\rangle \chi_{\mathcal{O}_{s} \mathcal{O}_{s}}^{(0)}, \\
\pi_{l_{s} l_{s^{\prime}}}=\left.\frac{-T}{2 N} \sum_{k} \operatorname{tr}\left\{\mathbf{G}_{k}[\mathbf{R}]^{-1} \mathbf{h}_{s}\left[\mathbf{R}^{\dagger}\right]^{-1} \mathbf{G}_{k}[\mathbf{R}]^{-1} \mathbf{h}_{s^{\prime}}\left[\mathbf{R}^{\dagger}\right]^{-1}\right\}\right|_{\xi=0} \\
=4\left\langle\tilde{\Lambda}_{l}^{2}\right\rangle_{\mathcal{O}_{s} \mathcal{O}_{s}}^{(0)},
\end{gathered}
$$

where we average the vertex over the Fermi surface:

$$
\begin{gathered}
\left\langle\tilde{\Lambda}_{r_{s}}\right\rangle=\frac{R_{0}^{2}}{2}\left\langle 2 \epsilon_{\mathbf{k}}\right\rangle_{\mathbf{k}_{F}}, \\
\left\langle\tilde{\Lambda}_{l_{s}}\right\rangle=\frac{1}{2} .
\end{gathered}
$$

We see that, after averaging all the vertices $\tilde{\Lambda}$ and selfenergy $\pi$ in Eq. (G7) over the Fermi surface, there are further cancellations in the denominator and the numerator of $\tilde{\Gamma}^{s}$ in Eq. (G7). Recasting Eq. (G7) in the form of Eq. (G2), we identify that the irreducible interaction (Landau parameter) $F_{s}$ in Eq. (G2) for each channel $s$ is

$F_{s}=-4\left\langle\tilde{\Lambda}_{r_{s}}\right\rangle^{2} \mathcal{D}_{0, r_{s} r_{s}}-8 \mathcal{D}_{0, r_{s} l_{s}}\left\langle\tilde{\Lambda}_{r_{s}}\right\rangle-4 \mathcal{D}_{0, l_{s} l_{s}}$.

\section{APPENDIX H: GAUGE INVARIANCE}

The RISB Lagrangian is invariant under the following gauge transformation [14,62]:

$$
\begin{array}{rlrl}
\Phi & \rightarrow \Phi U(\theta), & \boldsymbol{\Delta} & \rightarrow u^{t}(\theta) \boldsymbol{\Delta}\left[u^{\dagger}\right]^{t}(\theta), \\
\mathbf{R} & \rightarrow u^{\dagger}(\theta) \mathbf{R}, & \boldsymbol{\Lambda} & \rightarrow u^{\dagger}(\theta) \boldsymbol{\Lambda} u(\theta), \\
\mathbf{D} & \rightarrow u^{t}(\theta) \mathbf{D}, & \boldsymbol{\Lambda}^{c} \rightarrow u^{\dagger}(\theta) \boldsymbol{\Lambda}^{c} u(\theta),
\end{array}
$$

where

$$
\begin{gathered}
u(\theta)=e^{i \sum_{s} \theta_{s} \mathbf{T}_{s, a b},} \\
U(\theta)=e^{i \sum_{s} \theta_{s} \mathbf{T}_{s, a b} \Psi_{a}^{\dagger} \Psi_{b}} .
\end{gathered}
$$

Note that $\mathbf{T}_{s}$ are the generators for the gauge group, and $\theta_{s}$ are the Lie parameters. The specific form of $\mathbf{T}_{s}$ corresponding to our variational setup is shown in Appendix I.

We also define the corresponding gauge transformation for $\mathbf{x}$ [see Eq. (26)]:

$$
\mathbf{x} \rightarrow \mathcal{G}_{\theta}[\mathbf{x}]
$$

where the operator $\mathcal{G}_{\theta}$ transforms each element in Eq. (26) according to Eqs. (H1)-(H3).

\section{APPENDIX I: GAUGE-FIXING PROCEDURE}

In this section, we describe the gauge-fixing procedure for the fluctuation matrix $\mathcal{M}$. We define a gauge transformation [see Eq. (H6)]:

$$
\mathbf{x}^{\prime}=\mathcal{G}_{\theta}[\mathbf{x}(\xi)]=\mathbf{x}[\xi, \theta(\xi)],
$$

where each component of $\mathbf{x}$ transforms as

$$
\begin{array}{r}
r_{s}=\operatorname{Tr}\left[\tilde{\mathbf{h}}_{s}^{\dagger} u^{\dagger}(\theta) \mathbf{R}\right], \quad l_{s}=\operatorname{Tr}\left[\mathbf{h}_{s}^{\dagger} u^{\dagger}(\theta) \boldsymbol{\Lambda} u(\theta)\right], \\
D_{s}=\operatorname{Tr}\left[\tilde{\mathbf{h}}_{s}^{\dagger} u^{t}(\theta) \mathbf{D}\right], \quad l^{c}=\operatorname{Tr}\left[\mathbf{h}_{s}^{\dagger} u^{\dagger}(\theta) \boldsymbol{\Lambda}^{c} u(\theta)\right],
\end{array}
$$




$$
d_{s}=\operatorname{Tr}\left[\mathbf{h}_{s}^{\dagger} u^{t}(\theta) \boldsymbol{\Delta}\left[u^{\dagger}\right]^{t}(\theta)\right],
$$

and $\theta(0)=0$ at $\xi=0$.

Given that $\mathbf{x}$ is a solution of [see Eq. (D2)]

$$
\left.\sum_{\nu} \mathcal{M}_{\mu \nu} \frac{\partial x_{\nu}}{\partial \xi}\right|_{\xi=0}=\chi_{\mu}
$$

$\mathbf{x}^{\prime}$ is also a solution of

$$
\left.\sum_{\nu} \mathcal{M}_{\mu \nu} \frac{\partial x_{\nu}^{\prime}}{\partial \xi}\right|_{\xi=0}=\chi_{\mu} .
$$

Note again that here $\mu$ and $\nu$ run through all the elements in $\mathbf{x}$ [Eq. (26)], and we use the variational parameters as the subscripts. Also, we have

$$
\left.\frac{\partial x_{\mu}^{\prime}}{\partial \xi}\right|_{\xi=0}=\left.\frac{\partial x_{\mu}}{\partial \xi}\right|_{\xi=0}+\left.\left.\sum_{s} \frac{\partial x_{\mu}}{\partial \theta_{s}}\right|_{\theta=0} \frac{\partial \theta_{s}}{\partial \xi}\right|_{\xi=0} .
$$

Consequently, we show that

$$
\left.\left.\mathcal{M}_{\mu \nu} \frac{\partial x_{\nu}}{\partial \theta_{s}}\right|_{\theta=0} \frac{\partial \theta_{s}}{\partial \xi}\right|_{\xi=0}=0
$$

which implies that $\mathcal{M}_{\mu \nu}$ has zero eigenvalues, and the kernels are defined as

$$
K_{s, \mu}:=\left\{\left.\frac{\partial x_{\mu}}{\partial \theta_{s}}\right|_{\theta=0}\right\}
$$

such that

$$
M_{\mu \nu} K_{s, \nu}=0 \quad \forall K_{s} \in K .
$$

We can fix the gauge by projecting the matrices onto the vector space $v_{i, \mu}$ perpendicular to $K$, where $v_{i, \mu}$ can be constructed from the Gram-Schmidt process. The reduced fluctuation matrix and the embedding susceptibilities become

$$
\begin{gathered}
\overline{\mathcal{M}}_{i j}=v_{i, \mu} \mathcal{M}_{\mu \nu} v_{j, \nu}, \\
\bar{\chi}_{i \mathcal{O}}=v_{i, \mu} \chi_{\mu \mathcal{O}} .
\end{gathered}
$$

Consequently, we have the physical susceptibility

$$
\chi_{\mathcal{O O}}=\chi_{\mathcal{O O}}^{(0)}+\bar{\chi}_{i \mathcal{O}} \overline{\mathcal{M}}_{i j}^{-1} \bar{\chi}_{j \mathcal{O}}
$$

Now, the $\overline{\mathcal{M}}_{i j}^{-1}$ does not contain zero modes, and the matrix inversion is well defined.

For the model considered in this work, where we restricted the variational variables $\mathbf{x}$ to real numbers [Eqs. (21)-(25)], the $U(1)$ gauge degrees of freedom in the charge, spin, orbital, and spin-orbital channels are fixed. However, we are left with 1 gauge degree of freedom relating to the Nambu pseudospin rotation generator:

$$
\mathbf{T}=\tau_{1} \otimes \lambda_{6} \otimes\left(i \sigma_{y} \sigma_{z}\right),
$$

where $\tau_{i}$ is the Pauli matrix corresponding to the Nambu pseudospin. From the definition of the gauge transformation [Eqs. (H1)-(H3)], we derive the kernel $K$ :

$$
\begin{gathered}
\left.\frac{\partial r_{s}}{\partial \theta}\right|_{\theta=0}=-i \operatorname{Tr}\left[\left(\tilde{\mathbf{h}}_{s}\right)^{\dagger} \mathbf{T} \mathbf{R}\right]=\frac{r_{0}}{2 \sqrt{3}} \delta_{s, P}, \\
\left.\frac{\partial l_{s}}{\partial \theta}\right|_{\theta=0}=-i \operatorname{Tr}\left[\left(\mathbf{h}_{s}\right)^{\dagger}[\mathbf{T}, \boldsymbol{\Lambda}]\right]=-\frac{l_{0}}{\sqrt{3}} \delta_{s, P}, \\
\left.\frac{\partial d_{s}}{\partial \theta}\right|_{\theta=0}=i \operatorname{Tr}\left[\left(\mathbf{h}_{s}\right)^{\dagger}\left[\mathbf{T}^{t}, \boldsymbol{\Delta}\right]\right]=-\frac{d_{0}}{\sqrt{3}} \delta_{s, P}, \\
\left.\frac{\partial D_{s}}{\partial \theta_{j}}\right|_{\theta=0}=i \operatorname{Tr}\left[\left(\tilde{\mathbf{h}}_{s}\right)^{\dagger} \mathbf{T}^{t} \mathbf{D}\right]=\frac{D_{0}}{2 \sqrt{3}} \delta_{s, P}, \\
\left.\frac{\partial l_{s}^{c}}{\partial \theta}\right|_{\theta=0}=-i \operatorname{Tr}\left[\left(\mathbf{h}_{s}\right)^{\dagger}\left[\mathbf{T}, \boldsymbol{\Lambda}^{c}\right]\right]=-\frac{l_{0}^{c}}{\sqrt{3}} \delta_{s, P},
\end{gathered}
$$

where the $K$ vector is only nonzero in the pairing channel. We can then construct the vector space $v_{i, \mu}$ using the GramSchmidt process and compute the susceptibilities through Eqs. (I11)-(I13).

\section{APPENDIX J: VALIDITY OF THE FERMI-LIQUID APPROXIMATION}

In this section, we show the pairing susceptibility $\chi^{P}$ computed from the equation without enforcing the
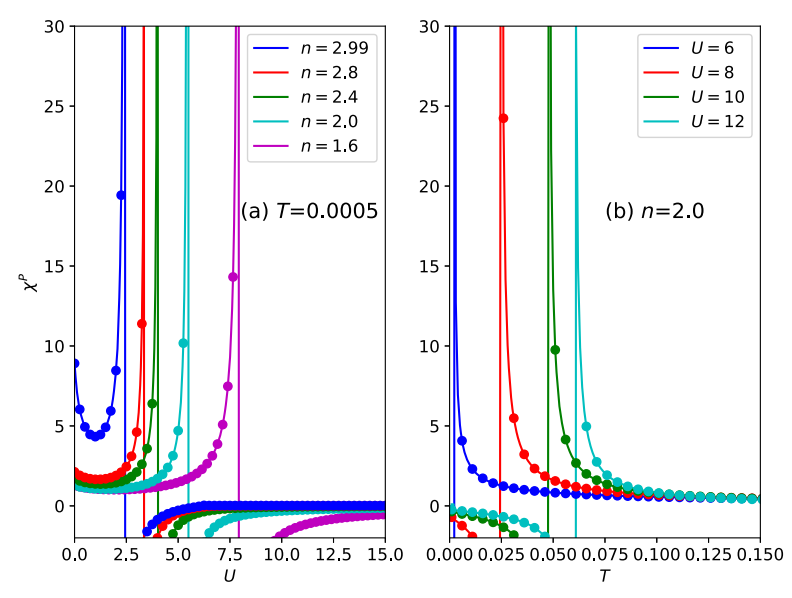

FIG. 9. Comparison of the pairing susceptibilities $\chi^{P}$ computed from Eq. (42) (solid line, without "quasiparticle constraint") and Eq. (56) (filled circles, with "quasiparticle constraint") for (a) $T=0.0005$ and $n=2.99,2.8,2.4,2.0,1.6$ as a function of $U$ and $J=U / 4$, and (b) $n=2.0$ and $U=6,8,10,12$ as a function of $T$ and $J=U / 4$. 


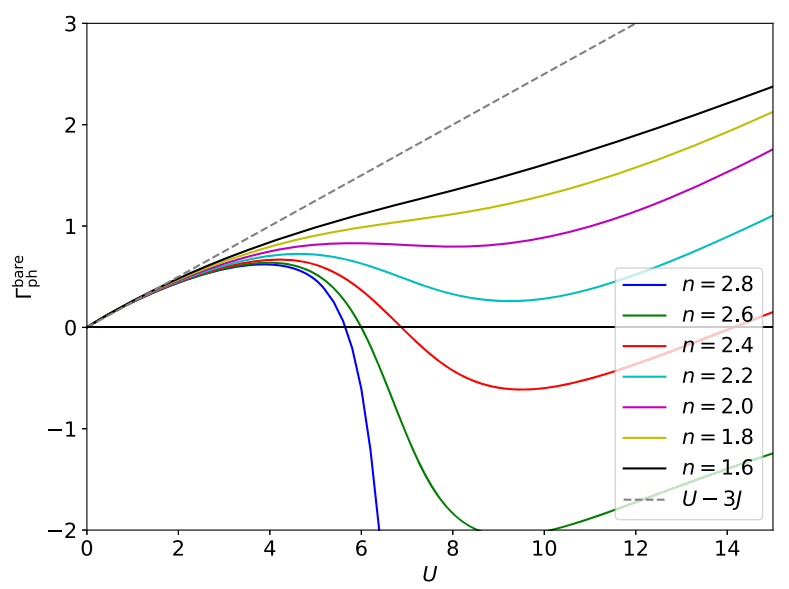

FIG. 10. Bare pairing interaction in the particle-hole channel $\Gamma_{\mathrm{ph}}^{\mathrm{bare}}[$ Eq. (K1)] as a function of Coulomb interaction $U$ and $J=$ $U / 4$ for filling $n=2.8,2.6,2.4,2.2,2.0,1.8,1.6$ at temperature $T=0.0005 t$.

quasiparticle constraint [Eq. (42)] and the equation with the quasiparticle constraint [Eq. (56)] in Fig. 9. The $\chi^{P}$ obtained from the two approaches are identical for all the parameter regime, indicating the validity of the Fermiliquid approximation described in Sec. IV.

\section{APPENDIX K: BARE PAIRING INTERACTION IN THE PARTICLE-HOLE CHANNEL}

We also compute the bare pairing interaction in the particle-hole channel defined as follows:

$\Gamma_{\mathrm{ph}}^{\mathrm{bare}}=\frac{1}{4}\left[F_{\mathrm{ch}}+F_{\mathrm{sp}}-F_{\mathrm{orb}}-F_{\mathrm{so}}-\frac{5}{3} F_{\mathrm{orb}^{*}}-\frac{5}{3} F_{\mathrm{so}^{*}}\right]$.

In this case, the summation of the fermionic particle-hole bubbles in Fig. 3(b) is ignored, and only the bare interaction (Landau parameters $F_{s}$ at $\mathbf{q}=0$ ) is considered. Figure 10 shows the bare pairing interaction in the particle-hole channel. We find that the bare pairing interaction only becomes negative (signalizing the pairing instability) for filling $n<2.3$.

\section{APPENDIX L: CONSISTENCY CHECK FOR SUSCEPTIBILITY}

We perform the consistency check for the pairing susceptibility between the one computed from the RISB fluctuation approach and the one computed from RISB mean-field theory with a small pairing field $\zeta$. The definition of the pairing susceptibility in the RISB self-consistent mean-field theory is $\chi^{P}=\left.\left(\partial\left\langle\mathcal{O}_{P}\right\rangle / \partial \zeta\right)\right|_{\zeta \rightarrow 0}$. The results from the two approaches are shown in Fig. 11(a) as a function of Coulomb interaction $U$ for $T=0.0005 t$ and filling $n=2.0$ and (b) as a function of temperature $T$ for $U=8 t$ and filling $n=2.0$. We confirm that the $\chi^{\mathrm{P}}(\mathbf{q}=0, \omega=0)$ computed from the
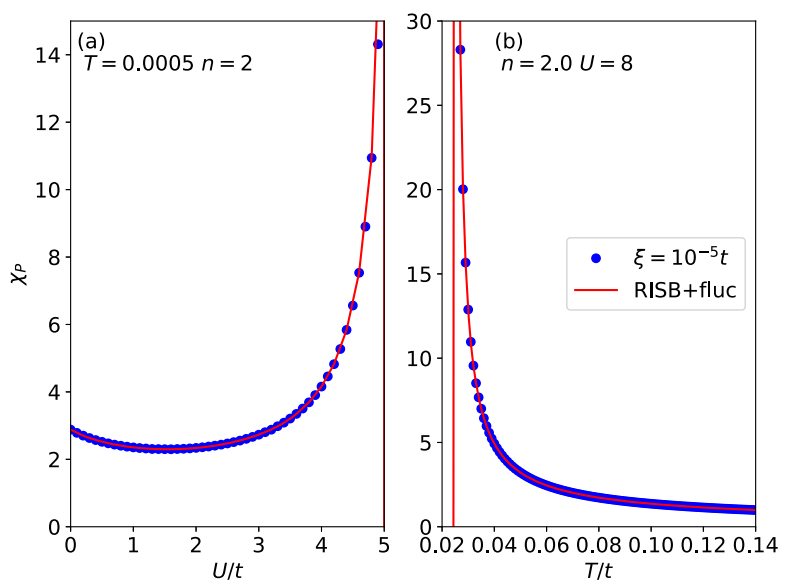

FIG. 11. Comparison of the uniform pairing susceptibility $\chi^{\mathrm{P}}(\mathbf{q}=0, \omega=0)$ evaluated from the fluctuation approach with the pairing susceptibility evaluated from the mean-field solution $\chi^{\mathrm{P}}=\left(d\left\langle\mathcal{O}_{\mathrm{P}}\right\rangle / d \zeta\right)$ with small pairing field $\zeta=10^{-5}$ for (a) temperature $T=0.0005 t$ and filling $n=2.0$ and (b) Coulomb interaction $U=8 t$ and filling $n=2.0$. We fix Hund's coupling interaction at $J=U / 4$.

fluctuation approach (red line) agrees excellently with the $\chi^{\mathrm{P}}$ computed from the mean-field theory with a small pairing field $\zeta=10^{-5} t$ (blue dots). The agreement between the two approaches indicates the consistency of our fluctuation approach within the RISB framework.

\section{APPENDIX M: TOTAL ENERGY AND WEAK- TO STRONG-COUPLING CROSSOVER}

We now discuss the total energy of the $s$-wave spin-triplet pairing state. Figures 12(a) and 12(b) show the kinetic energy gain $\Delta E_{\mathrm{k}}=E_{\mathrm{k}}^{N}-E_{\mathrm{k}}^{s c}$, the potential energy gain $\Delta E_{\mathrm{pot}}=$ $E_{\mathrm{pot}}^{N}-E_{\mathrm{pot}}^{s c}$, and the total energy gain $\Delta E_{\mathrm{tot}}=E_{\mathrm{tot}}^{N}-E_{\mathrm{tot}}^{s c}$ for
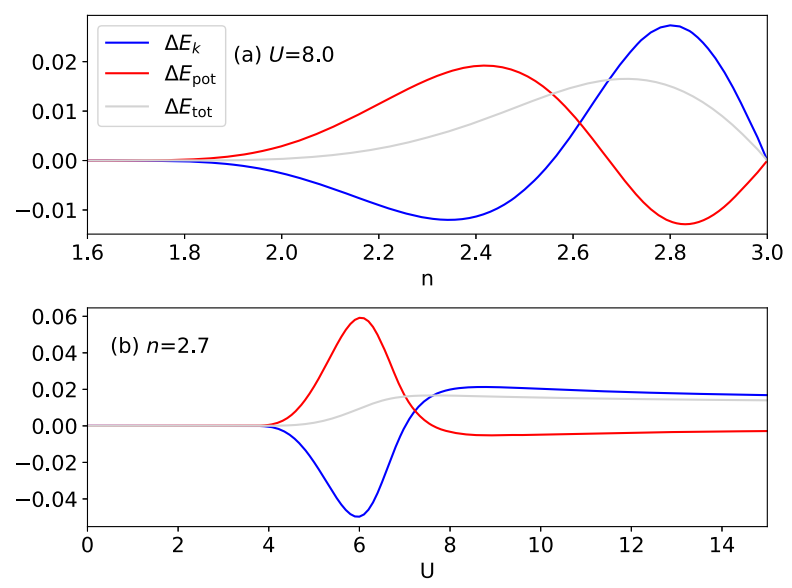

FIG. 12. Kinetic energy $\Delta E_{\mathrm{k}}$, potential energy $\Delta E_{\text {pot }}$, and total energy gain $\Delta E_{\text {tot }}$ for the superconducting paring state (a) as a function of electron filling $n$ at $U=8 t$ and $T=10^{-4} t$ and (b) as a function of Coulomb interaction $U$ and $J=U / 4$ at $n=2.7$ and $T=10^{-4} t$. 
forming the $s$-wave spin-triplet pairing state, where the superscripts $N$ and $s c$ correspond to the energy in the normal state and the superconducting state, respectively. The total energy in both Figs. 12(a) and 12(b) shows a typical weakcoupling to strong-coupling crossover behavior [71,92,93], where the energy gain is dominated by the potential energy in the weak-coupling limit and by the kinetic energy in the strong-coupling limit. Interestingly, we find that this crossover is located around Hund's metal crossover where the quasiparticle weight drops rapidly and the superconducting order parameter shows a pronounced peak.

\section{APPENDIX N: APPLICATION TO DENSITY MATRIX EMBEDDING THEORY}

In this section, we outline the equations for computing the susceptibility in the "noninteracting bath" DMET (NIBDMET) formalism. Since the NIB-DMET self-consistent equations can be reproduced from the RISB Lagrangian by enforcing $\mathbf{R}=I$ and an additional constraint in Eq. (44), the formalism in Sec. IV can be directly applied to NIBDMET by removing the $r_{s}$ sector of the fluctuation basis in Eq. (45), i.e., no fluctuation in R. Hence, the NIB-DMET fluctuation basis becomes

$$
\begin{gathered}
\mathbf{x}_{\mathbf{q}}=\left(l_{\mathrm{ch}, \mathbf{q}}, d_{\mathrm{ch}, \mathbf{q}}, D_{\mathrm{ch}, \mathbf{q}}, l_{\mathrm{ch}, \mathbf{q}}^{c}, \zeta_{\mathrm{ch}, \mathbf{q}}, \ldots, l_{s, \mathbf{q}}, d_{s, \mathbf{q}}, D_{s, \mathbf{q}},\right. \\
\left.l_{s, \mathbf{q}}^{c}, \zeta_{s, \mathbf{q}}, \ldots, l_{\mathrm{P}, \mathbf{q}}, d_{\mathrm{P}, \mathbf{q}}, D_{\mathrm{P}, \mathbf{q}}, l_{\mathrm{P}, \mathbf{q}}^{c}, \zeta_{\mathrm{P}, \mathbf{q}}\right),
\end{gathered}
$$

where, different from RISB [Eq. (45)], the variable $r_{s}$ is absent. Following the same derivation as in Sec. IV, the NIDMET susceptibility of an arbitrary operator $\hat{\mathcal{O}}$ has the following form:

$$
\chi_{\mathcal{O O}}(\mathbf{q})=\chi_{\mathcal{O O}}^{(0)}(\mathbf{q})+\sum_{\mu \nu} \chi_{\mu \mathcal{O}}(\mathbf{q}) \mathcal{M}_{\mu \nu}^{-1}(\mathbf{q}) \chi_{\nu \mathcal{O}}(\mathbf{q})
$$

where the fluctuation matrix $\mathcal{M}$ is given in Appendix $\mathrm{E}$ and we have to enforce $\mathbf{R}=I$ in each element. We have also introduced the following susceptibilities:

$$
\begin{gathered}
\chi_{\mathcal{O O}}^{(0)}(\mathbf{q})=-\frac{T}{2 N} \sum_{\omega_{n} \mathbf{k}} \operatorname{Tr}\left[\mathbf{G}_{\omega_{n}, \mathbf{k}+\mathbf{q}} \mathcal{O} \mathbf{G}_{\omega_{n}, \mathbf{k}} \mathcal{O}\right], \\
\chi_{\mu \mathcal{O}}(\mathbf{q})=\left.\frac{T}{2 N} \sum_{\omega_{n}, \mathbf{k}} \partial_{x_{\mu, \mathbf{q}}} \operatorname{Tr}\left[\mathbf{G}_{\omega_{n}, \mathbf{k}+\mathbf{q}, \mathbf{k}}[\xi, \mathbf{x}] \mathcal{O}\right]\right|_{[\xi=0, \mathbf{x}(\xi=0)]},
\end{gathered}
$$

where $\mathcal{O}$ is the single-particle matrix representation of a generic operator. The Green's function has the following form:

$$
\left[\mathbf{G}_{\omega_{n}, \mathbf{k}_{1}, \mathbf{k}_{2}}[\mathbf{x}, \xi]\right]^{-1}=i \omega_{n}-\left[H_{\mathbf{k}_{1} \mathbf{k}_{2}}^{\mathrm{qp}}\right]_{a b}+\xi_{\mathbf{k}_{1}-\mathbf{k}_{2}}[\mathcal{O}]_{a b},
$$

and $\mathbf{G}_{\omega_{n}, \mathbf{k}}$ is the Green's function evaluated at $\xi=0$. We also introduce the quasiparticle Hamiltonian (low-level mean-field Hamiltonian):

$$
\left[H_{\mathbf{k}_{1} \mathbf{k}_{2}}^{\mathrm{qp}}\right]_{a b} \equiv\left[\tilde{\mathbf{\epsilon}}_{\mathbf{k}_{1}}\right]_{a b} \delta_{\mathbf{k}_{1}, \mathbf{k}_{2}}+\left[\boldsymbol{\Lambda}_{\mathbf{k}_{1}-\mathbf{k}_{2}}\right]_{a b}
$$

where $\boldsymbol{\Lambda}$ corresponds to the correlation potential in NIB-DMET.

For the degenerate model considered in this work, the susceptibility can be written as

$\chi_{\mathcal{O}_{s} \mathcal{O}_{s}}(\mathbf{q})=\chi_{\mathcal{O}_{s} \mathcal{O}_{s}}^{(0)}(\mathbf{q})+\chi_{l_{s} \mathcal{O}_{s}}(\mathbf{q}) \mathcal{M}_{l_{s} l_{s}}^{-1}(\mathbf{q}) \chi_{l_{s} \mathcal{O}_{s}}(\mathbf{q})$,

where

$\chi_{l_{s} \mathcal{O}_{s}}(\mathbf{q})=-\frac{T}{2 N} \sum_{\omega_{n} \mathbf{k}} \operatorname{Tr}\left[\mathbf{G}_{\omega_{n}, \mathbf{k}+\mathbf{q}} \mathbf{h}_{s} \mathbf{G}_{\omega_{n}, \mathbf{k}} \mathbf{h}_{s}\right]=\chi_{\mathcal{O}_{s} \mathcal{O}_{s}}^{(0)}(\mathbf{q})$.

Here, $\mathcal{M}_{l_{s} l_{s}}^{-1}(\mathbf{q})$ denotes the $\mu=l_{s}$ and $\nu=l_{s}$ components of $\mathcal{M}_{\mu \nu}^{-1}(\mathbf{q})$, and $\mathbf{G}_{\omega_{n}, \mathbf{k}}=\left[i \omega_{n}-\tilde{\epsilon}_{\mathbf{k}}-\boldsymbol{\Lambda}\right]^{-1}$ is the saddlepoint Green's function.

Finally, we comment on the advantages and the disadvantages of RISB and NIB-DMET. One advantage of RISB with respect to NIB-DMET is the presence of the renormalization matrix $\mathbf{R}$. It allows the description of the Mott transition within the single-site approach [94], while in the standard NIB-DMET, one has to use at least a twosite cluster to capture the Mott transition [24]. On the other hand, the additional determination of $\mathbf{R}$ in RISB may require more self-consistency iterations with respect to NI-DMET, leading to more diagonalization of the embedding Hamiltonian $\hat{H}_{\text {emb }}$. Nevertheless, the performance and accuracy of the two methods are similar [26]. Note that our approach does not apply to the "interacting bath" construction of DMET (IB-DMET), which produces more accurate results than the NIB-DMET [24,85,95]. The extension of our approach to IB-DMET will be an interesting future topic.

[1] S. E. Barnes, New Method for the Anderson Model, J. Phys. F 6, 1375 (1976).

[2] P. Coleman, New Approach to the Mixed-Valence Problem, Phys. Rev. B 29, 3035 (1984).

[3] G. Kotliar and A. E. Ruckenstein, New Functional Integral Approach to Strongly Correlated Fermi Systems: The Gutzwiller Approximation as a Saddle Point, Phys. Rev. Lett. 57, 1362 (1986).

[4] T. Li, P. Wölfle, and P. J. Hirschfeld, Spin-RotationInvariant Slave-Boson Approach to the Hubbard Model, Phys. Rev. B 40, 6817 (1989).

[5] R. Frésard and P. Wölfle, Unified Slave Boson Representation of Spin and Charge Degrees of Freedom for Strongly Correlated Fermi Systems, Int. J. Mod. Phys. B 06, 685 (1992).

[6] S. Florens and A. Georges, Slave-Rotor Mean-Field Theories of Strongly Correlated Systems and the Mott Transition in Finite Dimensions, Phys. Rev. B 70, 035114 (2004). 
[7] L. de'Medici, A. Georges, and S. Biermann, OrbitalSelective Mott Transition in Multiband Systems: Slave-Spin Representation and Dynamical Mean-Field Theory, Phys. Rev. B 72, 205124 (2005).

[8] A. Georges, G. Kotliar, W. Krauth, and M. J. Rozenberg, Dynamical Mean-Field Theory of Strongly Correlated Fermion Systems and the Limit of Infinite Dimensions, Rev. Mod. Phys. 68, 13 (1996).

[9] F. Lechermann, A. Georges, G. Kotliar, and O. Parcollet, Rotationally Invariant Slave-Boson Formalism and Momentum Dependence of the Quasiparticle Weight, Phys. Rev. B 76, 155102 (2007).

[10] M. C. Gutzwiller, Effect of Correlation on the Ferromagnetism of Transition Metals, Phys. Rev. Lett. 10, 159 (1963).

[11] J. Bünemann and F. Gebhard, Equivalence of Gutzwiller and Slave-Boson Mean-Field Theories for Multiband Hubbard Models, Phys. Rev. B 76, 193104 (2007).

[12] C. Piefke and F. Lechermann, LDA + Slave-Boson Approach to the Correlated Electronic Structure of the Metamagnetic Bilayer Ruthenate $\mathrm{Sr}_{3} \mathrm{Ru}_{2} \mathrm{O}_{7}$, Physica Status Solidi (b) 248, 2269 (2011).

[13] C. Piefke and F. Lechermann, Rigorous Symmetry Adaptation of Multiorbital Rotationally Invariant Slave-Boson Theory with Application to Hund's Rules Physics, Phys. Rev. B 97, 125154 (2018).

[14] N. Lanatà, Y. Yao, X. Deng, V. Dobrosavljević, and G. Kotliar, Slave Boson Theory of Orbital Differentiation with Crystal Field Effects: Application to $\mathrm{UO}_{2}$, Phys. Rev. Lett. 118, 126401 (2017).

[15] N. Lanatà, T.-H. Lee, Y.-X. Yao, V. Stevanović, and V. Dobrosavljević, Connection between Mott Physics and Crystal Structure in a Series of Transition Metal Binary Compounds, npj Comput. Mater. 5, 30 (2019).

[16] L. de' Medici, J. Mravlje, and A. Georges, Janus-Faced Influence of Hund's Rule Coupling in Strongly Correlated Materials, Phys. Rev. Lett. 107, 256401 (2011).

[17] L. de' Medici, G. Giovannetti, and M. Capone, Selective Mott Physics as a Key to Iron Superconductors, Phys. Rev. Lett. 112, 177001 (2014).

[18] J. I. Facio, J. Mravlje, L. Pourovskii, P. S. Cornaglia, and V. Vildosola, Spin-Orbit and Anisotropic Strain Effects on the Electronic Correlations in $\mathrm{Sr}_{2} \mathrm{RuO}_{4}$, Phys. Rev. B 98, 085121 (2018).

[19] M. E. Barber, F. Lechermann, S. V. Streltsov, S. L. Skornyakov, S. Ghosh, B. J. Ramshaw, N. Kikugawa, D. A. Sokolov, A. P. Mackenzie, C. W. Hicks, and I. I. Mazin, Role of Correlations in Determining the Van Hove Strain in $\mathrm{Sr}_{2} \mathrm{RuO}_{4}$, Phys. Rev. B 100, 245139 (2019).

[20] N. Lanatà, Y.-X. Yao, C.-Z. Wang, K.-M. Ho, J. Schmalian, K. Haule, and G. Kotliar, $\gamma-\alpha$ Isostructural Transition in Cerium, Phys. Rev. Lett. 111, 196801 (2013).

[21] N. Lanatà, Y. Yao, C.-Z. Wang, K.-M. Ho, and G. Kotliar, Phase Diagram and Electronic Structure of Praseodymium and Plutonium, Phys. Rev. X 5, 011008 (2015).

[22] F. Lu, J. Zhao, H. Weng, Z. Fang, and X. Dai, Correlated Topological Insulators with Mixed Valence, Phys. Rev. Lett. 110, 096401 (2013).

[23] G. Kotliar, S. Y. Savrasov, K. Haule, V. S. Oudovenko, O. Parcollet, and C. A. Marianetti, Electronic Structure
Calculations with Dynamical Mean-Field Theory, Rev. Mod. Phys. 78, 865 (2006).

[24] G. Knizia and G. K.-L. Chan, Density Matrix Embedding: A Simple Alternative to Dynamical Mean-Field Theory, Phys. Rev. Lett. 109, 186404 (2012).

[25] T. Ayral, T.-H. Lee, and G. Kotliar, Dynamical Mean-Field Theory, Density-Matrix Embedding Theory, and Rotationally Invariant Slave Bosons: A Unified Perspective, Phys. Rev. B 96, 235139 (2017).

[26] T.-H. Lee, T. Ayral, Y.-X. Yao, N. Lanata, and G. Kotliar, Rotationally Invariant Slave-Boson and Density Matrix Embedding Theory: Unified Framework and Comparative Study on the One-Dimensional and Two-Dimensional Hubbard Model, Phys. Rev. B 99, 115129 (2019).

[27] C. Sun, U. Ray, Z.-H. Cui, M. Stoudenmire, M. Ferrero, and G. K.-L. Chan, Finite-Temperature Density Matrix Embedding Theory, Phys. Rev. B 101, 075131 (2020).

[28] N. Lanatà, X. Deng, and G. Kotliar, Finite-Temperature Gutzwiller Approximation from the Time-Dependent Variational Principle, Phys. Rev. B 92, 081108 (2015).

[29] M. Schiró and M. Fabrizio, Time-Dependent Mean Field Theory for Quench Dynamics in Correlated Electron Systems, Phys. Rev. Lett. 105, 076401 (2010).

[30] J. S. Kretchmer and G. K.-L. Chan, A Real-Time Extension of Density Matrix Embedding Theory for Non-equilibrium Electron Dynamics, J. Chem. Phys. 148, 054108 (2018).

[31] H.-Z. Ye, H. K. Tran, and T. Van Voorhis, Accurate Electronic Excitation Energies in Full-Valence Active Space via Bootstrap Embedding, J. Chem. Theory Comput. 17, 3335 (2021).

[32] P. V. Sriluckshmy, M. Nusspickel, E. Fertitta, and G. H. Booth, Fully Algebraic and Self-Consistent Effective Dynamics in a Static Quantum Embedding, Phys. Rev. B 103, 085131 (2021).

[33] N. Lanatà, T.-H. Lee, Y.-X. Yao, and V. Dobrosavljević, Emergent Bloch Excitations in Mott Matter, Phys. Rev. B 96, 195126 (2017).

[34] D. J. Scalapino, A Common Thread: The Pairing Interaction for Unconventional Superconductors, Rev. Mod. Phys. 84, 1383 (2012).

[35] N. Read and D. M. Newns, On the Solution of the CoqblinSchreiffer Hamiltonian by the Large-N Expansion Technique, J. Phys. C 16, 3273 (1983).

[36] L. Lilly, A. Muramatsu, and W. Hanke, Slave-Boson Mean Field Versus Quantum Monte Carlo Results for the Hubbard Model, Phys. Rev. Lett. 65, 1379 (1990).

[37] T. Jolicoeur and J. C. Le Guillou, Fluctuations Beyond the Gutzwiller Approximation in the Slave-Boson Approach, Phys. Rev. B 44, 2403 (1991).

[38] M. Lavagna, Functional-Integral Approach to Strongly Correlated Fermi Systems: Quantum Fluctuations Beyond the Gutzwiller Approximation, Phys. Rev. B 41, 142 (1990).

[39] T. Li, Y. S. Sun, and P. Wölfle, Dynamic Response Functions of Hubbard Model in Gutzwiller Approximation, Z. Phys. B 82, 369 (1991).

[40] E. Arrigoni and G. C. Strinati, Exact Criterion for Choosing the Hopping Operator in the Four-Slave-Boson Approach, Phys. Rev. B 52, 13707 (1995). 
[41] R. Raimondi and C. Castellani, Lower and Upper Hubbard Bands: A Slave-Boson Treatment, Phys. Rev. B 48, 11453 (1993).

[42] W. Zimmermann, R. Frésard, and P. Wölfle, Spin and Charge Structure Factor of the Two-Dimensional Hubbard Model, Phys. Rev. B 56, 10097 (1997).

[43] V. H. Dao and R. Frésard, Collective Modes in the Paramagnetic Phase of the Hubbard Model, Phys. Rev. B 95, 165127 (2017).

[44] D. Riegler, M. Klett, T. Neupert, R. Thomale, and P. Wölfle, Slave-Boson Analysis of the Two-Dimensional Hubbard Model, Phys. Rev. B 101, 235137 (2020).

[45] G. Seibold and J. Lorenzana, Time-Dependent Gutzwiller Approximation for the Hubbard Model, Phys. Rev. Lett. 86, 2605 (2001).

[46] G. Seibold, F. Becca, P. Rubin, and J. Lorenzana, TimeDependent Gutzwiller Theory of Magnetic Excitations in the Hubbard Model, Phys. Rev. B 69, 155113 (2004).

[47] M. Fabrizio, Quantum Fluctuations Beyond the Gutzwiller Approximation, Phys. Rev. B 95, 075156 (2017).

[48] G. Seibold, F. Becca, and J. Lorenzana, Theory of Antibound States in Partially Filled Narrow Band Systems, Phys. Rev. Lett. 100, 016405 (2008).

[49] G. Seibold, F. Becca, and J. Lorenzana, Time-Dependent Gutzwiller Theory of Pairing Fluctuations in the Hubbard Model, Phys. Rev. B 78, 045114 (2008).

[50] E. von Oelsen, G. Seibold, and J. Büemann, Time-Dependent Gutzwiller Theory for Multi-band Hubbard Models, New J. Phys. 13, 113031 (2011).

[51] E. v. Oelsen, G. Seibold, and J. Bünemann, Time-Dependent Gutzwiller Theory for Multiband Hubbard Models, Phys. Rev. Lett. 107, 076402 (2011).

[52] P. Werner, E. Gull, M. Troyer, and A. J. Millis, Spin Freezing Transition and Non-Fermi-Liquid Self-Energy in a Three-Orbital Model, Phys. Rev. Lett. 101, 166405 (2008).

[53] S. Hoshino and P. Werner, Superconductivity from Emerging Magnetic Moments, Phys. Rev. Lett. 115, 247001 (2015).

[54] M. Zegrodnik, J. Spałek, and J. Bünemann, Coexistence of Spin-Triplet Superconductivity with Magnetism within a Single Mechanism for Orbitally Degenerate Correlated Electrons: Statistically Consistent Gutzwiller Approximation, New J. Phys. 15, 073050 (2013).

[55] M. Zegrodnik, J. Bünemann, and J. Spałek, Even-Parity Spin-Triplet Pairing by Purely Repulsive Interactions for Orbitally Degenerate Correlated Fermions, New J. Phys. 16, 033001 (2014).

[56] M. Capone, M. Fabrizio, C. Castellani, and E. Tosatti, Strongly Correlated Superconductivity, Science 296, 2364 (2002).

[57] M. Capone, M. Fabrizio, and E. Tosatti, Direct Transition between a Singlet Mott Insulator and a Superconductor, Phys. Rev. Lett. 86, 5361 (2001).

[58] J. Kanamori, Electron Correlation and Ferromagnetism of Transition Metals, Prog. Theor. Phys. 30, 275 (1963).

[59] A. K. C. Cheung and D. F. Agterberg, Superconductivity in the Presence of Spin-Orbit Interactions Stabilized by Hund Coupling, Phys. Rev. B 99, 024516 (2019).
[60] H. G. Suh, H. Menke, P. M. R. Brydon, C. Timm, A. Ramires, and D.F. Agterberg, Stabilizing Even-Parity Chiral Superconductivity in $\mathrm{Sr}_{2} \mathrm{RuO}_{4}$, Phys. Rev. Research 2, 032023(R) (2020).

[61] S.-O. Kaba and D. Sénéchal, Group-Theoretical Classification of Superconducting States of Strontium Ruthenate, Phys. Rev. B 100, 214507 (2019).

[62] A. Isidori and M. Capone, Rotationally Invariant Slave Bosons for Strongly Correlated Superconductors, Phys. Rev. B 80, 115120 (2009).

[63] G. Kotliar and J. Liu, Superconducting Instabilities in the Large-U Limit of a Generalized Hubbard Model, Phys. Rev. Lett. 61, 1784 (1988).

[64] M. Grilli and G. Kotliar, Fermi-Liquid Parameters and Superconducting Instabilities of a Generalized t-J Model, Phys. Rev. Lett. 64, 1170 (1990).

[65] M. Grilli, R. Raimondi, C. Castellani, C. Di Castro, and G. Kotliar, Superconductivity, Phase Separation, and ChargeTransfer Instability in the $\mathrm{U}=\infty$ Limit of the Three-Band Model of the $\mathrm{CuO}_{2}$ Planes, Phys. Rev. Lett. 67, 259 (1991).

[66] A. Sudb $\varnothing$ and A. Houghton, Charge Fluctuations: Spin Fluctuations and Superconductivity in a $\mathrm{CuO}_{2}$ Sheet, Phys. Rev. B 42, 4105 (1990).

[67] A. Georges, L. de' Medici, and J. Mravlje, Strong Correlations from Hund's Coupling, Annu. Rev. Condens. Matter Phys. 4, 137 (2013).

[68] A. Isidori, M. Berović, L. Fanfarillo, L. de' Medici, M. Fabrizio, and M. Capone, Charge Disproportionation, Mixed Valence, and Janus Effect in Multiorbital Systems: A Tale of Two Insulators, Phys. Rev. Lett. 122, 186401 (2019).

[69] J. E. Han, Spin-Triplet s-wave Local Pairing Induced by Hund's Rule Coupling, Phys. Rev. B 70, 054513 (2004).

[70] W. Metzner and D. Vollhardt, Correlated Lattice Fermions in $d=\infty$ Dimensions, Phys. Rev. Lett. 62, 324 (1989).

[71] M. Bak and R. Micnas, Superconducting Properties of the Attractive Hubbard Model in the Slave-Boson Approach, J. Phys. Condens. Matter 10, 9029 (1998).

[72] L. de' Medici, Hund's Induced Fermi-Liquid Instabilities and Enhanced Quasiparticle Interactions, Phys. Rev. Lett. 118, 167003 (2017).

[73] C. M. Puetter and H.-Y. Kee, Identifying Spin-Triplet Pairing in Spin-Orbit Coupled Multi-Band Superconductors, Europhys. Lett. 98, 27010 (2012).

[74] A. W. Lindquist and H.-Y. Kee, Distinct Reduction of Knight Shift in Superconducting State of $\mathrm{Sr}_{2} \mathrm{RuO}_{4}$ under Uniaxial Strain, Phys. Rev. Research 2, 032055(R) (2020).

[75] J. Clepkens, A. W. Lindquist, and H.-Y. Kee, Shadowed Triplet Pairings in Hund's Metals with Spin-Orbit Coupling, arXiv:2009.08597.

[76] O. Vafek and A. V. Chubukov, Hund Interaction, Spin-Orbit Coupling, and the Mechanism of Superconductivity in Strongly Hole-Doped Iron Pnictides, Phys. Rev. Lett. 118, 087003 (2017).

[77] P. Coleman, Y. Komijani, and E. J. König, Triplet Resonating Valence Bond State and Superconductivity in Hund's Metals, Phys. Rev. Lett. 125, 077001 (2020).

[78] S. Raghu, X.-L. Qi, C. Honerkamp, and S.-C. Zhang, Topological Mott Insulators, Phys. Rev. Lett. 100, 156401 (2008). 
[79] M. Dzero, K. Sun, V. Galitski, and P. Coleman, Topological Kondo Insulators, Phys. Rev. Lett. 104, 106408 (2010).

[80] M. Klett, S. Ok, D. Riegler, P. Wölfle, R. Thomale, and T. Neupert, Topology and Magnetism in the Kondo Insulator Phase Diagram, Phys. Rev. B 101, 161112 (2020).

[81] P. Zhang, K. Yaji, T. Hashimoto, Y. Ota, T. Kondo, K. Okazaki, Z. Wang, J. Wen, G. D. Gu, H. Ding, and S. Shin, Observation of Topological Superconductivity on the Surface of an Iron-Based Superconductor, Science $\mathbf{3 6 0}$, 182 (2018).

[82] W. Liu, L. Cao, S. Zhu, L. Kong, G. Wang, M. Papaj, P. Zhang, Y.-B. Liu, H. Chen, G. Li, F. Yang, T. Kondo, S. Du, G.-H. Cao, S. Shin, L. Fu, Z. Yin, H.-J. Gao, and H. Ding, A New Majorana Platform in an Fe-As Bilayer Superconductor, Nat. Commun. 11, 5688 (2020).

[83] G. Rohringer, H. Hafermann, A. Toschi, A. A. Katanin, A. E. Antipov, M. I. Katsnelson, A. I. Lichtenstein, A. N. Rubtsov, and K. Held, Diagrammatic Routes to Nonlocal Correlations Beyond Dynamical Mean Field Theory, Rev. Mod. Phys. 90, 025003 (2018).

[84] B. Senjean, N. Nakatani, M. Tsuchiizu, and E. Fromager, Site-Occupation Embedding Theory Using Bethe Ansatz Local Density Approximations, Phys. Rev. B 97, 235105 (2018).

[85] I. W. Bulik, G. E. Scuseria, and J. Dukelsky, Density Matrix Embedding from Broken Symmetry Lattice Mean Fields, Phys. Rev. B 89, 035140 (2014).

[86] S. Sekaran, M. Tsuchiizu, M. Saubanère, and E. Fromager, Householder Transformed Density Matrix Functional Embedding Theory, arXiv:2103.04194.
[87] M. Fabrizio, Gutzwiller Description of Non-Magnetic Mott Insulators: Dimer Lattice Model, Phys. Rev. B 76, 165110 (2007).

[88] N. Lanatà, P. Barone, and M. Fabrizio, Superconductivity in the Doped Bilayer Hubbard Model, Phys. Rev. B 80, 224524 (2009).

[89] N. Lanatà, H. U. R. Strand, X. Dai, and B. Hellsing, Efficient Implementation of the Gutzwiller Variational Method, Phys. Rev. B 85, 035133 (2012).

[90] J. Rogers, T.-H. Lee, S. Pakdel, W. Xu, V. Dobrosavljević, Y.-X. Yao, O. Christiansen, and N. Lanatà, Bypassing the Computational Bottleneck of Quantum-Embedding Theories for Strong Electron Correlations with Machine Learning, Phys. Rev. Research 3, 013101 (2021).

[91] B.-X. Zheng, C.-M. Chung, P. Corboz, G. Ehlers, M.-P. Qin, R. M. Noack, H. Shi, S. R. White, S. Zhang, and G. K.-L. Chan, Stripe Order in the Underdoped Region of the TwoDimensional Hubbard Model, Science 358, 1155 (2017).

[92] J. O. Sofo and C. A. Balseiro, Slave-Boson Mean-Field Theory for the Negative-U Hubbard Model, Phys. Rev. B 45, 377 (1992).

[93] B. R. Bulka and S. Robaszkiewicz, Superconducting Properties of the Attractive Hubbard Model: A Slave-Boson Study, Phys. Rev. B 54, 13138 (1996).

[94] W. F. Brinkman and T. M. Rice, Application of Gutzwiller's Variational Method to the Metal-Insulator Transition, Phys. Rev. B 2, 4302 (1970).

[95] M. Kawano and C. Hotta, Comparative Study of the Density Matrix Embedding Theory for Hubbard Models, Phys. Rev. B 102, 235111 (2020). 